RIKEN-BNL preprint

\title{
Forming Disoriented Chiral Condensates through Fluctuations
}

\author{
Dirk H. Rischke \\ RIKEN-BNL Research Center, Physics Department \\ Brookhaven National Laboratory, Upton, New York 11973, U.S.A.
}

\begin{abstract}
Using the influence functional formalism, classical equations of motion for the $O(N)$ model are derived in the presence of a heat bath, in both the symmetric phase as well as the phase of spontaneously broken symmetry. The heat bath leads to dissipation and fluctuation terms in the classical equations of motion, which are explicitly computed to lowest order in perturbation theory. In the broken phase these terms are found to be large for the $\sigma$ field, even at zero temperature, due to the decay process $\sigma \rightarrow \pi \pi$, while they are small for the $\pi$ fields at temperatures below $T_{c} \simeq 160 \mathrm{MeV}$. It is shown that in large volumes the presence of dissipation and fluctuations suppresses the formation of disoriented chiral condensates (DCC's). In small volumes, however, fluctuations become sufficiently large to induce the formation of DCC's even if chiral symmetry has not been restored in the initial stage of the system's evolution.
\end{abstract}

PACS number(s): 25.75.-q, 11.30.Qc, 11.30.Rd, 12.39.Fe, 12.38.Mh

\section{INTRODUCTION AND CONCLUSIONS}

At vanishing net-baryon number density and temperatures above $T_{c} \simeq 160 \mathrm{MeV}$, lattice calculations of quantum chromodynamics (QCD) predict the existence of a phase of nuclear matter where quarks and gluons are deconfined and chiral symmetry is restored [1]. One of the primary goals of relativistic heavy-ion physics is to create and study this phase in nuclear collisions [2].

The formation of a so-called disoriented chiral condensate (DCC) has been proposed as a possible signature for the restoration of chiral symmetry [3]. The idea is the following: in the phase where chiral symmetry is restored the quark condensate vanishes, $\langle\bar{q} q\rangle \simeq 0$. If at all, in a heavy-ion collision this state can only be transiently created. Once the system cools below $T_{c}$, chiral symmetry is spontaneously broken and the system has to evolve back into the true ground state where $\langle\bar{q} q\rangle \neq 0$. If in the course of this evolution the pseudoscalar condensate $\left\langle\bar{q} \boldsymbol{\tau} \gamma_{5} q\right\rangle$ assumes non-vanishing values (instead of remaining zero, as in the ground state), one speaks of a disoriented chiral condensate.

This mechanism becomes physically most transparent in the framework of the $O(4)$ model. One identifies $\phi_{1} \sim\langle\bar{q} q\rangle, \phi_{i} \sim\left\langle\bar{q} \tau_{i} \gamma_{5} q\right\rangle, i=2,3,4$, and spontaneously broken symmetry is realized by a potential $U(\phi)$ which looks like a (tilted) "mexican hat", with minimum at $\phi=\left(f_{\pi}, \mathbf{0}\right)$ for $T=0$. For increasing $T$, the "hat" becomes shallower and the minimum moves towards the origin, such that $\phi \rightarrow 0$ for $T \rightarrow T_{c}$, and chiral symmetry is restored. The creation of DCC's is most likely in the so-called "quench scenario" 軘. Here, it is assumed that, after restoration of chiral symmetry, the system cools instantaneously to $T=0$. If the fields $\phi$ are assumed to follow classical equations of motion in the potential $U(\phi)$, the evolution of the system can be visualized as "rolling down" from the initial state with restored chiral symmetry, $\underline{\phi}=0$, into the true ground state $\phi=\left(f_{\pi}, \mathbf{0}\right)$. If that happens on a "path" where $\phi_{i} \neq 0, i=2,3,4$, the chiral condensate becomes "disoriented".

The ratio $R$ of neutral pions to the sum of neutral and charged pions was suggested as experimental observable [3]. If a single domain of DCC is formed, the probability $P(R) \sim 1 / \sqrt{R}$, which is drastically 
different from the case where pions are emitted in a statistically independent manner, $P(R) \sim \delta(R-1 / 3)$ (for large, isospin-symmetric systems).

The formation and decay of DCC's has been studied in a variety of approaches over the last couple of years [5], and the original idea has undergone several refinements. One obvious effect that has an influence on DCC formation in heavy-ion collisions is the presence of a background of a multitude of other particles. Most of these are pions with typical transverse momenta on the order of a couple of hundred MeV [6]. In [7] it was assumed that these pions constitute a background ("heat bath") of unobserved, thermalized degrees of freedom. Their presence leads to temperature-dependent dissipation and fluctuation terms in the classical equations of motion which correspond to four-particle interactions between classical fields and particles in the heat bath and can be rigorously derived 8] (cf. also [9]) via the influence functional formalism [10]. For a typical (average) temperature evolution in a heavy-ion collision it was then studied in [7], how DCC's form in an expanding system which is in contact with this (steadily cooling) heat bath of particles. The main result was that, on the average, dissipation and fluctuation tend to suppress the formation of DCC's. However, fluctuations grow $\sim 1 / \sqrt{V}$ for $V \rightarrow 0$. Thus, in small volumes and in a single event, the fluctuations can be large enough to destabilize the system and actually enhance the likelihood to form a DCC.

The authors of [7] made two approximations. The first was to compute the dissipation terms in the chirally symmetric phase and then to use them for the evolution of the system in the phase where chiral symmetry is spontaneously broken. This leads to considerable simplifications, since in the symmetric phase these terms are straightforward generalizations of results obtained previously in $\phi^{4}$ theory [8]. For dissipation arising from four-particle interactions which are present both in the symmetric and the broken phase, this approximation is probably justified in the initial stage of the evolution, where temperatures are high and close to $T_{c}$. It becomes questionable at smaller temperatures due to the fact that not all particles have the same mass in the broken phase (the $\sigma$ is heavy, while the $\pi$ 's are light).

There is, however, another reason to reconsider this approximation at temperatures below $T_{c}$. In the broken phase the structure of the underlying Lagrangian is fundamentally different: there are additional three-particle interactions. As will be shown in detail in the following, this has the consequence that, while the dissipation is $\sim \lambda^{2}$ in the symmetric phase and of equal magnitude for all fields $\phi_{a}, a=1, \ldots, 4$, dissipative corrections arise already to first order in $\lambda$ in the broken phase, and are of sizable magnitude for the $\sigma$ degree of freedom and rather small for pions. The former correspond physically to the decay of a $\sigma$ into two $\pi$ 's (cf. also [11]), and are non-vanishing even at $T=0$. Therefore, the dissipation coefficients below $T_{c}$ are different from an extrapolation of the results obtained in the symmetric phase.

The second approximation made in [7] was to infer the variance of the fluctuation terms $\xi_{a}, a=1, \ldots, 4$, from the dissipation coefficient $\eta$ via

$$
\left\langle\xi_{a}(t) \xi_{b}\left(t^{\prime}\right)\right\rangle=\frac{2 T \eta}{V} \delta\left(t-t^{\prime}\right) \delta_{a b} .
$$

Apart from the fact that this equation does not account for different dissipation coefficients for $\sigma$ and $\pi$ 's, one has to note that the factor $2 T$ stems from the high-temperature (i.e. classical) limit of a more general expression (cf. [8] and below). For temperatures $T$ smaller than the typical mass scale of the theory, i.e., for $T<m_{\pi} \simeq T_{c}$, one therefore expects sizable deviations from (iR). In particular, whenever $\eta$ happens to be finite at $T=0$ (which, as mentioned above and shown below, is indeed the case for the $\sigma$ field in the broken phase), eq. (1) predicts that fluctuations vanish even in the presence of dissipation, in contradiction to the dissipation-fluctuation theorem.

The aim of this paper is to make a first step towards a consistent treatment of dissipation and fluctuation in the framework of the $O(4)$ model in the phase where chiral symmetry is broken. The outline of the paper, as well as the main results and conclusions are as follows. In section II, the derivation of the influence functional is presented for a system of $N$ real-valued scalar fields $\phi_{1}, \ldots, \phi_{N}$. Short wavelength modes, i.e., those with "hard" momenta $|\mathbf{k}|>k_{c}$, where $k_{c}$ is an arbitrary momentum scale, are separated from long wavelength modes, i.e., those with "soft" momenta $|\mathbf{k}| \leq k_{c}$ [12], and the reduced density matrix for the soft modes is obtained from the full density matrix by tracing over the hard degrees of freedom, which thus are thought to constitute the unobserved background ("heat bath") mentioned above. The influence functional enters as a phase factor in the reduced density matrix. This section is a straightforward generalization of the treatment in [8]. It serves merely to introduce the notation, and can be skipped by readers familiar with the subject.

Section III contains the derivation of the classical equations of motion for the soft fields by expanding the reduced density matrix around its diagonal elements. The main difference as compared to previous treatments of the subject 88,13,14 is that functional derivatives of the influence functional are expressed as 
averages over functional derivatives of the action characterizing the interaction between soft, classical fields and the unobserved hard degrees of freedom. This has the advantage that, in a perturbative computation of the correction terms to the classical equations of motion up to some given order $n$ in the coupling constant, expectation values of the hard degrees of freedom have to computed only to order $n-1$. It is furthermore shown that for systems with more than one field degree of freedom, there can be cross correlations between the noise terms for different fields.

In Section IV, the general framework derived in the previous sections is applied to the $O(N)$ model in the symmetric case. The corrections to the classical equation of motion are first computed to order $\lambda$, where they only change the mass term in the classical equation of motion, and then also to order $\lambda^{2}$, where they lead to dissipation and fluctuations. The treatment is fairly cursory, since this case is rather similar to $\phi^{4}$ theory (or, in other words, the $O(1)$ case) discussed in detail in [8]. The main focus is to demonstrate the applicability of the method developed in Section III. It is shown that the damping coefficient agrees with previous results in the cases $N=1[\beta]$ and $N=4$ [7].

In Section $\mathrm{V}$ the $O(N)$ model is discussed for the case of spontaneously broken symmetry. In this case, only corrections up to first order in the coupling constant are considered, but due to the presence of two interaction vertices in the Lagrangian (one proportional to $\lambda$ and the other proportional to $\lambda f_{\pi} \sim \lambda^{1 / 2}$ ), there is dissipation and fluctuation already to this order in $\lambda$. In particular, the dissipation coefficient for the $\sigma$ field is shown to be large even at $T=0, \eta_{\sigma} \simeq m_{\sigma} \simeq 600 \mathrm{MeV}$. The physical process responsible for this is the decay of a $\sigma$ into two $\pi$ 's. This has important consequences which are discussed in Section VI. On the other hand, the dissipation coefficient for the $\pi$ fields is small for the temperature range of interest, the reason being that scattering of a $\pi$ or $\sigma$ from the heat bath off a classical $\pi$ field is strongly suppressed by phase space. For $T \rightarrow 0$, as well as in the chiral limit $m_{\pi} \rightarrow 0$ for arbitrary $T$, one even has $\eta_{\pi} \rightarrow 0$. The classical equations of motion for $\sigma$ 's and $\pi$ 's are derived and studied in detail for the $\mathbf{k}=0$ modes of the classical fields. It is shown that the static solution for the $\sigma$ field corresponds to the well-known shift of the vacuum ground state at finite temperature. Moreover, the validity of Goldstone's theorem at the classical level is checked.

In Section VI, arguments are presented that the formation of DCC's is most likely in a quench scenario, i.e., at $T=0$. Numerical solutions of the equations of motion for the homogeneous modes of the fields at zero temperature are then presented, which show that, in large volumes, the large dissipation coefficient for the $\sigma$ field leads to a rapid damping of oscillations of all classical fields, including the pions. The formation of DCC's seems thus not very likely in large systems. In small volumes, on the other hand, the fluctuations associated with the dissipation are large enough to disorient the pion fields and possibly lead to the formation of DCC's. These results are in agreement with those found in [7], except that here they apply even at $T=0$. This may have the experimentally interesting implication that DCC's are perhaps formed more readily in collisions of lighter ions, or even in $p p$-collisions (which constitutes a possible explanation for the CENTAURO events 15), while they are presumably less likely to be formed in collisions of heavy ions. A definite conclusion, however, can only be drawn after performing dynamical simulations including modes with finite $\mathbf{k}$ and taking the overall expansion of the system into account [16].

There is, however, another possible consequence of the results found here. As long as the volume is small, $\sim 10 \mathrm{fm}^{3}$, the disorientation of the $\pi$ fields (and possibly DCC formation) becomes likely even if the system's evolution starts near the true ground state (even small perturbations in the $\pi$ fields suffice). As a consequence, in small systems restoration of chiral symmetry does not seem to be a necessary prerequisite to observe disorientation of classical pion fields. It is, however, rather likely that this fluctuation-induced phenomenon is related to ordinary fluctuations in finite volumes.

The results of the present work have to be viewed in the light of the following two comments: (a) the classical approximation works well in the limit of large occupation numbers. For instance, in thermodynamical equilibrium this is achieved for modes with energy $\omega \ll T$. However, the lowest-energy mode for (non-interacting) $\sigma$ particles has $\omega=m_{\sigma} \simeq 600 \mathrm{MeV}$ which is much larger than the temperature in the broken phase $\left(T \leq T_{c} \simeq 160 \mathrm{MeV}\right)$. Therefore, at least in thermodynamical equilibrium, the $\sigma$ field should not seriously be considered classically. In a sense, the expected large quantum corrections become manifest in the fluctuations induced by the decay $\sigma \rightarrow \pi \pi$ as calculated in the present work. (b) The dissipation and fluctuation terms are here computed to first order in perturbation theory. However, for realistic parameters of the $O(N)$ model the coupling constant $\lambda \simeq 20$, which renders the perturbative expansion uncontrollable. Future studies will have to improve on this point.

Possible other extensions of the present work are (a) the inclusion of collisional interactions between $\sigma$ 's and $\pi$ 's which are of order $\sim \lambda^{2}$ [17], (b) the study of long wavelength modes with finite momenta $\mathbf{k}$ instead of the homogeneous modes only, as well as (c) the study of DCC formation including these effects with a 
realistic temperature evolution 16 .

Units are $\hbar=c=k_{B}=1$, and the metric tensor is $g^{\mu \nu}=\operatorname{diag}(+,-,-,-)$.

\section{DERIVATION OF THE INFLUENCE FUNCTIONAL}

Let us consider a quantum system of $N$ real-valued scalar fields $\underline{\phi}=\left(\phi_{1}, \ldots, \phi_{N}\right)$, characterised by a Lagrangian density $\mathcal{L}(\underline{\phi})$. The Hamilton operator $\hat{H} \equiv \int \mathrm{d}^{3} \mathbf{x} \mathcal{H}(\hat{\phi}(x)), \mathcal{H} \equiv \underline{\pi} \cdot \partial_{t} \underline{\phi}-\mathcal{L}, \underline{\pi} \equiv \partial \mathcal{L} / \partial\left(\partial_{t} \underline{\phi}\right)$, is assumed to have no explicit time dependence. The time evolution of the density matrix reads:

$$
i \partial_{t} \hat{\rho}=[\hat{H}, \hat{\rho}],
$$

with initial condition $\hat{\rho}\left(t_{i}\right) \equiv \hat{\rho}_{i}$. The formal solution at time $t_{f}$ is:

$$
\hat{\rho}\left(t_{f}\right)=\hat{U}\left(t_{f}, t_{i}\right) \hat{\rho}_{i} \hat{U}\left(t_{i}, t_{f}\right),
$$

where

$$
\hat{U}\left(t_{f}, t_{i}\right) \equiv \exp \left\{-i \hat{H}\left(t_{f}-t_{i}\right)\right\} \equiv \hat{U}^{\dagger}\left(t_{i}, t_{f}\right) \equiv \hat{U}^{-1}\left(t_{i}, t_{f}\right)
$$

is the time evolution operator. Let us choose a basis $\left\{\left|\underline{\phi}_{f}\right\rangle\right\}$ of eigenfunctions of the Schrödinger field operator $\hat{\phi}_{f}(\mathbf{x})$. In this basis, one finds for the density matrix element

$$
\rho\left(\underline{\phi}_{f}, \underline{\phi}_{f}^{\prime} ; t_{f}\right) \equiv\left\langle\underline{\phi}_{f}\left|\hat{\rho}\left(t_{f}\right)\right| \underline{\phi}_{f}^{\prime}\right\rangle=\int \mathrm{D} \underline{\phi}_{i} \mathrm{D} \underline{\phi}_{i}^{\prime} \rho\left(\underline{\phi}_{i}, \underline{\phi}_{i}^{\prime} ; t_{i}\right) \int_{\underline{\phi}_{i}}^{\underline{\phi}_{f}} \mathcal{D} \underline{\phi} \int_{\underline{\phi}_{i}^{\prime}}^{\underline{\phi}_{f}^{\prime}} \mathcal{D} \underline{\phi}^{\prime} \exp \left\{i\left(S[\underline{\phi}]-S\left[\underline{\phi}^{\prime}\right]\right)\right\}
$$

where one has employed eq. (3), the completeness relation

$$
1=\int \mathrm{D} \underline{\phi}_{i}\left|\underline{\phi}_{i}\right\rangle\left\langle\underline{\phi}_{i}\right|
$$

(here $\left.\mathrm{D} \underline{\phi} \equiv \prod_{a=1}^{N} \prod_{\mathbf{x}} \mathrm{d} \phi_{a}(\mathbf{x})\right)$, and the path integral representation

$$
\left\langle\underline{\phi}_{f}\left|\hat{U}\left(t_{f}, t_{i}\right)\right| \underline{\phi}_{i}\right\rangle \equiv \int_{\underline{\phi}_{i}}^{\underline{\phi}_{f}} \mathcal{D} \underline{\phi} \exp \{i S[\underline{\phi}]\}
$$

where $\mathcal{D} \underline{\phi} \equiv \prod_{a=1}^{N} \prod_{t, \mathbf{x}} \mathrm{d} \phi_{a}(t, \mathbf{x})$, and $S[\underline{\phi}]=\int_{t_{i}}^{t_{f}} \mathrm{~d} t \int \mathrm{d}^{3} \mathbf{x} \mathcal{L}(\phi(t, \mathbf{x})) \equiv \int_{t_{i}}^{t_{f}} \mathrm{~d}^{4} x \mathcal{L}(\underline{\phi}(x))$. Note that it is customary [8,13,14] to employ the closed-time-path formalism [18] to simplify the right-hand side of eq. (5)). Although this is an elegant bookkeeping device, for the sake of clarity we shall continue to work in terms of the fields $\phi$ and $\phi^{\prime}$.

At each time $\bar{t}$, a particular field component with $3-$ momentum $\mathbf{k}$ has the Fourier representation

$$
\underline{\phi}(t, \mathbf{k}) \equiv \int \mathrm{d}^{3} \mathbf{x} e^{-i \mathbf{k} \cdot \mathbf{x}} \underline{\phi}(t, \mathbf{x}) .
$$

Let us now separate "hard" from "soft" degrees of freedom [8, 12]. More precisely, let us define soft fields

$$
\underline{\varphi}(t, \mathbf{x}) \equiv \int \frac{\mathrm{d}^{3} \mathbf{k}}{(2 \pi)^{3}} e^{i \mathbf{k} \cdot \mathbf{x}} \underline{\phi}(t, \mathbf{k}) \Theta\left(k_{c}-|\mathbf{k}|\right),
$$

and hard fields

$$
\underline{\Phi}(t, \mathbf{x}) \equiv \int \frac{\mathrm{d}^{3} \mathbf{k}}{(2 \pi)^{3}} e^{i \mathbf{k} \cdot \mathbf{x}} \underline{\phi}(t, \mathbf{k}) \Theta\left(|\mathbf{k}|-k_{c}\right),
$$

where $k_{c}$ is an arbitrary momentum scale separating hard from soft momentum modes. Obviously, the space spanned by $\{|\underline{\phi}\rangle$ is the product space spanned by $\{|\underline{\varphi}\rangle\}$ and $\{|\underline{\Phi}\rangle\},\{\mid \underline{\phi}\}=\{|\underline{\varphi}\rangle\} \otimes\{|\underline{\Phi}\rangle\}$. (This is most 
easily seen in the space of functions $\underline{\phi}(t, \mathbf{k})$.) Also, $\underline{\phi}(x)=\underline{\varphi}(x)+\underline{\Phi}(x)$, and $S[\underline{\phi}]=S[\underline{\varphi}]+S[\underline{\Phi}]+S_{I}[\underline{\varphi}, \underline{\Phi}]$, where $S_{I}[\underline{\varphi}, \underline{\Phi}]$ is the action characterizing interactions between soft and hard fields.

The density matrix (5) then assumes the form

$$
\begin{aligned}
& \rho\left(\underline{\varphi}_{f}, \underline{\Phi}_{f} ; \underline{\varphi}_{f}^{\prime}, \underline{\Phi}_{f}^{\prime} ; t_{f}\right)=\int \mathrm{D} \underline{\varphi}_{i} \mathrm{D} \underline{\Phi}_{i} \mathrm{D} \underline{\varphi}_{i}^{\prime} \mathrm{D} \underline{\Phi}_{i}^{\prime} \rho\left(\underline{\varphi}_{i}, \underline{\Phi}_{i} ; \underline{\varphi}_{i}^{\prime}, \underline{\Phi}_{i}^{\prime} ; t_{i}\right) \\
& \times \int_{\underline{\varphi}_{i}}^{\underline{\varphi}_{f}} \mathcal{D} \underline{\varphi} \int_{\underline{\Phi}_{i}}^{\underline{\Phi}_{f}} \mathcal{D} \underline{\Phi} \int_{\underline{\varphi}_{i}^{\prime}}^{\underline{\varphi}_{f}^{\prime}} \mathcal{D} \underline{\varphi}^{\prime} \int_{\underline{\Phi}_{i}^{\prime}}^{\underline{\Phi}_{f}^{\prime}} \mathcal{D} \underline{\Phi}^{\prime} \exp \left\{i\left(S[\underline{\varphi}]+S[\underline{\Phi}]+S_{I}[\underline{\varphi}, \underline{\Phi}]-S\left[\underline{\varphi}^{\prime}\right]-S\left[\underline{\Phi}^{\prime}\right]-S_{I}\left[\underline{\varphi}^{\prime}, \underline{\Phi}^{\prime}\right]\right)\right\} .
\end{aligned}
$$

Let us now assume that the interactions between soft and hard fields vanish at the initial time $t_{i}$. Then, the initial density matrix $\hat{\rho}_{i}$ is block-diagonal, $\hat{\rho}_{i}=\hat{\rho}_{i}^{(\varphi)} \otimes \hat{\rho}_{i}^{(\Phi)}$, and

$$
\rho\left(\underline{\varphi}_{i}, \underline{\Phi}_{i} ; \underline{\varphi}_{i}^{\prime}, \underline{\Phi}_{i}^{\prime} ; t_{i}\right) \equiv \rho^{(\varphi)}\left(\underline{\varphi}_{i}, \underline{\varphi}_{i}^{\prime} ; t_{i}\right) \rho^{(\Phi)}\left(\underline{\Phi}_{i}, \underline{\Phi}_{i}^{\prime} ; t_{i}\right) .
$$

The object of interest in the following is the reduced density matrix $\rho^{(\varphi)}\left(\underline{\varphi}_{f}, \underline{\varphi}_{f}^{\prime} ; t_{f}\right)$ for the soft fields, which is obtained by tracing (11) over the degrees of freedom of the hard fields. This reduced density matrix has the form

$$
\rho^{(\varphi)}\left(\underline{\varphi}_{f}, \underline{\varphi}_{f}^{\prime} ; t_{f}\right)=\int \mathrm{D} \underline{\varphi}_{i} \mathrm{D} \underline{\varphi}_{i}^{\prime} \rho^{(\varphi)}\left(\underline{\varphi}_{i}, \underline{\varphi}_{i}^{\prime} ; t_{i}\right) \int_{\underline{\varphi}_{i}}^{\underline{\varphi}_{f}} \mathcal{D} \underline{\varphi} \int_{\underline{\varphi}_{i}^{\prime}}^{\underline{\varphi}_{f}^{\prime}} \mathcal{D} \underline{\varphi}^{\prime} \exp \left\{i\left(S[\underline{\varphi}]-S\left[\underline{\varphi}^{\prime}\right]+S_{I F}\left[\underline{\varphi}, \underline{\varphi}^{\prime}\right]\right)\right\},
$$

where the influence functional $S_{I F}\left[\underline{\varphi}, \underline{\varphi^{\prime}}\right]$ is defined by $[10]$

$$
\begin{aligned}
& \exp \left\{i S_{I F}\left[\underline{\varphi}, \underline{\varphi}^{\prime}\right]\right\} \\
& \equiv \int \mathrm{D} \underline{\Phi}_{f} \mathrm{D} \underline{\Phi}_{i} \mathrm{D} \underline{\Phi}_{i}^{\prime} \rho^{(\Phi)}\left(\underline{\Phi}_{i}, \underline{\Phi}_{i}^{\prime} ; t_{i}\right) \int_{\underline{\Phi}_{i}}^{\underline{\Phi}_{f}} \mathcal{D} \underline{\Phi} \int_{\underline{\Phi}_{i}^{\prime}}^{\underline{\Phi}_{f}} \mathcal{D} \underline{\Phi}^{\prime} \exp \left\{i\left(S[\underline{\Phi}]+S_{I}[\underline{\varphi}, \underline{\Phi}]-S\left[\underline{\Phi}^{\prime}\right]-S_{I}\left[\underline{\varphi}^{\prime}, \underline{\Phi}^{\prime}\right]\right)\right\} .
\end{aligned}
$$

An obvious property of the influence functional (for real-valued scalar fields) is

$$
S_{I F}\left[\underline{\varphi}, \underline{\varphi^{\prime}}\right]=-S_{I F}^{*}\left[\underline{\varphi}^{\prime}, \underline{\varphi}\right] .
$$

For the proof, note that for any element of the (hermitean) density matrix one has $\rho^{*}\left(\phi, \phi^{\prime} ; t\right) \equiv$ $\left\langle\phi|\hat{\rho}(t)| \phi^{\prime}\right\rangle^{*}=\left\langle\phi^{\prime}\left|\hat{\rho}^{\dagger}(t)\right| \phi\right\rangle \equiv\left\langle\phi^{\prime}|\hat{\rho}(t)| \phi\right\rangle \equiv \rho\left(\phi^{\prime}, \phi ; t\right)$.

Another property is

$$
S_{I F}[\underline{\varphi}, \underline{\varphi}]=0 .
$$

For the proof, note that in $S_{I F}, \varphi$ plays the role of an external, and thus fixed, field. Defining the action $S_{\varphi}[\underline{\Phi}] \equiv S[\underline{\Phi}]+S_{I}[\underline{\varphi}, \underline{\Phi}]$ for hard fields in the presence of this external field $\underline{\varphi}$, and a corresponding time evolution operator $\hat{\hat{U}}_{\varphi}\left(t_{f}, t_{i}\right)$ with matrix elements

$$
\left\langle\underline{\Phi}_{f}\left|\hat{U}_{\varphi}\left(t_{f}, t_{i}\right)\right| \underline{\Phi}_{i}\right\rangle \equiv \int_{\underline{\Phi}_{i}}^{\underline{\Phi}_{f}} \mathcal{D} \underline{\Phi} \exp \left\{i S_{\varphi}[\underline{\Phi}]\right\},
$$

one then reverts in eq. (14) the steps which led to eq. (5). Then, $\exp \left\{i S_{I F}[\underline{\varphi}, \underline{\varphi}]\right\}=\int \mathrm{D} \underline{\Phi}_{f} \rho_{\varphi}^{(\Phi)}\left(\underline{\Phi}_{f}, \underline{\Phi}_{f} ; t_{f}\right) \equiv$ $\operatorname{Tr} \hat{\rho}_{\varphi}^{(\Phi)}\left(t_{f}\right) \equiv 1$, consequently, $S_{I F}[\underline{\varphi}, \underline{\varphi}] \equiv 0$. Here, $\hat{\rho}_{\varphi}^{(\Phi)}\left(t_{f}\right)$ is the density matrix for the hard degrees of freedom as it evolved from its initial value $\hat{\rho}_{i}^{(\Phi)}$ to the final time $t_{f}$ subject to the time evolution operator $\hat{U}_{\varphi}\left(t_{f}, t_{i}\right)$. This evolution in general differs from the one when the external field $\underline{\varphi}$ is absent.

\section{DERIVATION OF CLASSICAL EQUATIONS OF MOTION FOR THE SOFT FIELDS}

The classical equations of motion for the soft fields $\underline{\underline{\varphi}}$ are determined by expanding the phase $S[\underline{\varphi}]-S\left[\varphi^{\prime}\right]+$ $S_{I F}\left[\underline{\varphi}, \underline{\varphi}^{\prime}\right]$ in eq. (13) around field configurations $\underline{\varphi} \equiv \underline{\varphi^{\prime}}$, i.e., where this phase vanishes according to (16)). Let us introduce 


$$
\underline{\bar{\varphi}} \equiv \frac{1}{2}\left(\underline{\varphi}+\underline{\varphi}^{\prime}\right) \quad, \quad \Delta \underline{\varphi} \equiv \underline{\varphi}-\underline{\varphi}^{\prime},
$$

and expand $S[\underline{\varphi}]-S\left[\underline{\varphi}^{\prime}\right]+S_{I F}\left[\underline{\varphi}, \underline{\varphi}^{\prime}\right]$ to quadratic order in $\Delta \underline{\varphi}$ :

$$
\begin{aligned}
& S[\underline{\varphi}]-S\left[\underline{\varphi}^{\prime}\right]+S_{I F}\left[\underline{\varphi}, \underline{\varphi^{\prime}}\right] \\
& \simeq \int_{t_{i}}^{t_{f}} \mathrm{~d}^{4} x\left[\frac{\delta S[\underline{\bar{\varphi}}]}{\delta \underline{\bar{\varphi}}(x)}+\frac{1}{2}\left(\frac{\delta S_{I F}\left[\underline{\varphi}, \underline{\varphi^{\prime}}\right]}{\delta \underline{\varphi}(x)}-\frac{\delta S_{I F}\left[\underline{\varphi}, \underline{\varphi^{\prime}}\right]}{\delta \underline{\varphi^{\prime}}(x)}\right)_{\underline{\varphi}=\underline{\varphi}^{\prime}=\underline{\varphi}}\right] \cdot \Delta \underline{\varphi}(x)+\frac{1}{8} \int_{t_{i}}^{t_{f}} \mathrm{~d}^{4} x \mathrm{~d}^{4} y \\
& \times \sum_{a, b=1}^{N} \Delta \varphi_{a}(x)\left(\frac{\delta^{2} S_{I F}\left[\underline{\varphi}, \underline{\varphi^{\prime}}\right]}{\delta \varphi_{a}(x) \delta \varphi_{b}(y)}-\frac{\delta^{2} S_{I F}\left[\underline{\varphi}, \underline{\varphi}^{\prime}\right]}{\delta \varphi_{a}(x) \delta \varphi_{b}^{\prime}(y)}-\frac{\delta^{2} S_{I F}\left[\underline{\varphi}, \underline{\varphi}^{\prime}\right]}{\delta \varphi_{a}^{\prime}(x) \delta \varphi_{b}(y)}+\frac{\delta^{2} S_{I F}\left[\underline{\varphi}, \underline{\varphi}^{\prime}\right]}{\delta \varphi_{a}^{\prime}(x) \delta \varphi_{b}^{\prime}(y)}\right)_{\underline{\varphi}=\underline{\varphi}^{\prime}=\underline{\bar{\varphi}}} \Delta \varphi_{b}(y) .
\end{aligned}
$$

Previous derivations of classical equations of motion in a background of hard fields usually compute the functional derivatives of the influence functional in eq. (19) directly for the specific system under consideration. However, these functional derivatives can be further evaluated also in the general case. To this end, let us define an " $n$-point function":

$$
A\left(\underline{\Phi}, \underline{\Phi}^{\prime}\right) \equiv \Phi_{a_{1}}^{n_{1}}\left(x_{1}\right) \Phi_{a_{2}}^{n_{2}}\left(x_{2}\right) \cdots \Phi_{a_{k}}^{n_{k}}\left(x_{k}\right) \Phi_{b_{1}}^{\prime m_{1}}\left(x_{1}^{\prime}\right) \Phi_{b_{2}}^{\prime m_{2}}\left(x_{2}^{\prime}\right) \cdots \Phi_{b_{l}}^{\prime m_{l}}\left(x_{l}^{\prime}\right), \quad n=\sum_{i=1}^{k} n_{i}+\sum_{j=1}^{l} m_{j},
$$

of the fields $\underline{\Phi}, \underline{\Phi}^{\prime}$. Here, $a_{i}$ and $b_{j}$ label components of these $N$-dimensional fields. Without loss of generality one may assume that $t_{1} \geq t_{2} \geq \cdots$ and $t_{1}^{\prime} \geq t_{2}^{\prime} \geq \cdots$. Let us then define the average of $A\left(\underline{\Phi}, \underline{\Phi}^{\prime}\right)$ in the presence of the "background field" $\underline{\bar{\varphi}}$ as:

$$
\begin{aligned}
\left\langle A\left(\underline{\Phi}, \underline{\Phi}^{\prime}\right)\right\rangle_{\bar{\varphi}} & \equiv \int \mathrm{D} \underline{\Phi}_{f} \mathrm{D} \underline{\Phi}_{i} \mathrm{D} \underline{\Phi}_{i}^{\prime} \rho^{(\Phi)}\left(\underline{\Phi}_{i}, \underline{\Phi}_{i}^{\prime} ; t_{i}\right) \\
& \times \int_{\underline{\Phi}_{i}}^{\underline{\Phi}_{f}} \mathcal{D} \underline{\Phi} \int_{\underline{\Phi}_{i}^{\prime}}^{\underline{\Phi}_{f}} \mathcal{D} \underline{\Phi}^{\prime} \exp \left\{i\left(S[\underline{\Phi}]+S_{I}[\underline{\bar{\varphi}}, \underline{\Phi}]-S\left[\underline{\Phi}^{\prime}\right]-S_{I}\left[\underline{\bar{\varphi}}, \underline{\Phi}^{\prime}\right]\right)\right\} A\left(\underline{\Phi}, \underline{\Phi}^{\prime}\right) .
\end{aligned}
$$

Using the time evolution operator (17) (with $\underline{\varphi} \equiv \underline{\bar{\varphi}}$ ), one notices that this is equivalent to

$$
\left\langle A\left(\underline{\Phi}, \underline{\Phi}^{\prime}\right)\right\rangle_{\bar{\varphi}} \equiv \operatorname{Tr}\left\{\hat{\rho}_{\bar{\varphi}}^{(\Phi)}\left(t_{f}\right) A\left(\underline{\hat{\Phi}}, \underline{\Phi}^{\prime}\right)\right\},
$$

i.e., the usual expectation value of the $n$-point function $A$ in the (in general, non-equilibrium) ensemble characterized by the density matrix $\hat{\rho}_{\bar{\varphi}}^{(\Phi)}\left(t_{f}\right)$. Note that in this expectation value all $\underline{\Phi}$ fields are timeordered, while all $\Phi^{\prime}$ fields are anti-time-ordered.

Writing $S_{I F}=-i \ln \exp \left\{i S_{I F}\right\}$, one now observes with the definition (14) and the property (16) of the influence functional, and the definition of the average (21) that

$$
\begin{aligned}
& \left.\frac{\delta S_{I F}\left[\underline{\varphi}, \underline{\varphi}^{\prime}\right]}{\delta \underline{\varphi}(x)}\right|_{\underline{\varphi}=\underline{\varphi^{\prime}=\underline{\varphi}}}=\left\langle\frac{\delta S_{I}[\underline{\bar{\varphi}}, \underline{\Phi}]}{\delta \underline{\bar{\varphi}}(x)}\right\rangle_{\underline{\underline{\varphi}}} \\
& \left.\frac{\delta S_{I F}\left[\underline{\varphi}, \underline{\varphi^{\prime}}\right]}{\delta \underline{\varphi}^{\prime}(x)}\right|_{\underline{\varphi}=\underline{\varphi}^{\prime}=\underline{\bar{\varphi}}}=-\left\langle\frac{\delta S_{I}\left[\underline{\bar{\varphi}}, \underline{\Phi}^{\prime}\right]}{\delta \underline{\bar{\varphi}}(x)}\right\rangle_{\underline{\underline{\varphi}}}, \\
& \left.\frac{\delta^{2} S_{I F}\left[\underline{\varphi}, \underline{\varphi}^{\prime}\right]}{\delta \varphi_{a}(x) \delta \varphi_{b}(y)}\right|_{\underline{\varphi}=\underline{\varphi}^{\prime}=\underline{\varphi}}=\left\langle\frac{\delta^{2} S_{I}[\underline{\bar{\varphi}}, \underline{\Phi}]}{\delta \bar{\varphi}_{a}(x) \delta \bar{\varphi}_{b}(y)}\right\rangle_{\underline{\bar{\varphi}}} \\
& +i\left\langle\frac{\delta S_{I}[\underline{\bar{\varphi}}, \underline{\Phi}]}{\delta \bar{\varphi}_{a}(x)} \frac{\delta S_{I}[\underline{\bar{\varphi}}, \underline{\Phi}]}{\delta \bar{\varphi}_{b}(y)}\right\rangle_{\underline{\underline{\varphi}}}-i\left\langle\frac{\delta S_{I}[\underline{\bar{\varphi}}, \underline{\Phi}]}{\delta \bar{\varphi}_{a}(x)}\right\rangle_{\underline{\underline{\varphi}}}\left\langle\frac{\delta S_{I}[\underline{\bar{\varphi}}, \underline{\Phi}]}{\delta \bar{\varphi}_{b}(y)}\right\rangle_{\underline{\underline{\varphi}}}, \\
& \left.\frac{\delta^{2} S_{I F}\left[\underline{\underline{\varphi}}, \underline{\varphi}^{\prime}\right]}{\delta \varphi_{a}(x) \delta \varphi_{b}^{\prime}(y)}\right|_{\underline{\varphi}=\underline{\varphi}^{\prime}=\underline{\bar{\varphi}}}=-i\left\langle\frac{\delta S_{I}[\underline{\bar{\varphi}}, \underline{\Phi}]}{\delta \bar{\varphi}_{a}(x)} \frac{\delta S_{I}\left[\underline{\bar{\varphi}}, \underline{\Phi}^{\prime}\right]}{\delta \bar{\varphi}_{b}(y)}\right\rangle_{\underline{\underline{\varphi}}}+i\left\langle\frac{\delta S_{I}[\underline{\bar{\varphi}}, \underline{\Phi}]}{\delta \bar{\varphi}_{a}(x)}\right\rangle_{\underline{\underline{\varphi}}}\left\langle\frac{\delta S_{I}\left[\underline{\bar{\varphi}}, \underline{\Phi}^{\prime}\right]}{\delta \bar{\varphi}_{b}(y)}\right\rangle_{\underline{\bar{\varphi}}},
\end{aligned}
$$




$$
\begin{aligned}
\left.\frac{\delta^{2} S_{I F}\left[\underline{\underline{\varphi}}, \underline{\varphi}^{\prime}\right]}{\delta \varphi_{a}^{\prime}(x) \delta \varphi_{b}^{\prime}(y)}\right|_{\underline{\varphi}=\underline{\varphi}^{\prime}=\bar{\varphi}} & =-\left\langle\frac{\delta^{2} S_{I}\left[\underline{\bar{\varphi}}, \underline{\Phi}^{\prime}\right]}{\delta \bar{\varphi}_{a}(x) \delta \bar{\varphi}_{b}(y)}\right\rangle_{\underline{\underline{\varphi}}} \\
& +i\left\langle\frac{\delta S_{I}\left[\underline{\bar{\varphi}}, \underline{\Phi}^{\prime}\right]}{\delta \bar{\varphi}_{a}(x)} \frac{\delta S_{I}\left[\underline{\bar{\varphi}}, \underline{\Phi}^{\prime}\right]}{\delta \bar{\varphi}_{b}(y)}\right\rangle_{\underline{\underline{\varphi}}}-i\left\langle\frac{\delta S_{I}\left[\overline{\underline{\varphi}}, \underline{\Phi}^{\prime}\right]}{\delta \bar{\varphi}_{a}(x)}\right\rangle_{\underline{\bar{\varphi}}}\left\langle\frac{\delta S_{I}\left[\overline{\bar{\varphi}}, \underline{\Phi}^{\prime}\right]}{\delta \bar{\varphi}_{b}(y)}\right\rangle_{\underline{\bar{\varphi}}}
\end{aligned}
$$

Since the fields $\underline{\bar{\varphi}}, \underline{\Phi}, \underline{\Phi}^{\prime}$ are assumed to be real-valued, eq. (19) can be further simplified using symmetry properties of the average (21) with respect to exchange of $\underline{\Phi} \leftrightarrow \underline{\Phi}^{\prime}$, for instance:

$$
\left\langle\frac{\delta S_{I}\left[\underline{\bar{\varphi}}, \underline{\Phi}^{\prime}\right]}{\delta \underline{\bar{\varphi}}(x)}\right\rangle_{\underline{\underline{\varphi}}} \equiv\left\langle\frac{\delta S_{I}[\underline{\bar{\varphi}}, \underline{\Phi}]}{\delta \underline{\bar{\varphi}}(x)}\right\rangle_{\underline{\varphi}}^{*}
$$

Then, eq. (19) becomes:

$$
S[\underline{\varphi}]-S\left[\underline{\varphi}^{\prime}\right]+S_{I F}\left[\underline{\varphi}, \underline{\varphi^{\prime}}\right] \simeq \int_{t_{i}}^{t_{f}} \mathrm{~d}^{4} x \underline{\mathcal{E}}(x) \cdot \Delta \underline{\varphi}(x)+\frac{i}{2} \int_{t_{i}}^{t_{f}} \mathrm{~d}^{4} x \mathrm{~d}^{4} y \sum_{a, b=1}^{N} \Delta \varphi_{a}(x) \mathcal{I}_{a b}(x, y) \Delta \varphi_{b}(y),
$$

where

$$
\begin{aligned}
\underline{\mathcal{E}}(x) & =\frac{\delta S[\underline{\bar{\varphi}}]}{\delta \underline{\bar{\varphi}}(x)}+\underline{\mathcal{R}}(x), \\
\underline{\mathcal{R}}(x) & \equiv \operatorname{Re}\left\langle\frac{\delta S_{I}[\underline{\bar{\varphi}}, \underline{\Phi}]}{\delta \underline{\bar{\varphi}}(x)}\right\rangle_{\underline{\underline{\varphi}}}, \\
\mathcal{I}_{a b}(x, y) & \equiv \frac{1}{2} \operatorname{Im}\left\langle\frac{\delta^{2} S_{I}[\underline{\bar{\varphi}}, \underline{\Phi}]}{\delta \bar{\varphi}_{a}(x) \delta \bar{\varphi}_{b}(y)}\right\rangle_{\underline{\underline{\varphi}}}-\operatorname{Re}\left\langle\frac{\delta S_{I}[\underline{\bar{\varphi}}, \underline{\Phi}]}{\delta \bar{\varphi}_{a}(x)}\right\rangle_{\underline{\underline{\varphi}}} \operatorname{Re}\left\langle\frac{\delta S_{I}[\overline{\underline{\varphi}}, \underline{\Phi}]}{\delta \bar{\varphi}_{b}(y)}\right\rangle_{\bar{\varphi}} \\
& +\frac{1}{4}\left\langle\left(\frac{\delta S_{I}[\underline{\bar{\varphi}}, \underline{\Phi}]}{\delta \bar{\varphi}_{a}(x)}+\frac{\delta S_{I}\left[\underline{\bar{\varphi}}, \underline{\Phi}^{\prime}\right]}{\delta \bar{\varphi}_{a}(x)}\right)\left(\frac{\delta S_{I}[\underline{\bar{\varphi}}, \underline{\Phi}]}{\delta \bar{\varphi}_{b}(y)}+\frac{\delta S_{I}\left[\underline{\bar{\varphi}}, \underline{\Phi}^{\prime}\right]}{\delta \bar{\varphi}_{b}(y)}\right)\right\rangle_{\underline{\bar{\varphi}}}
\end{aligned}
$$

are real-valued functions. Inserting this into expression (13) and changing the integration variables $\underline{\varphi}, \underline{\varphi^{\prime}}$ to $\underline{\bar{\varphi}}, \Delta \underline{\varphi}$, one obtains

$$
\begin{aligned}
& \rho^{(\varphi)}\left(\underline{\varphi}_{f}, \Delta \underline{\varphi}_{f} ; t_{f}\right) \simeq \int \mathrm{D} \underline{\underline{\varphi}}_{i} \mathrm{D} \Delta \underline{\varphi}_{i} \rho^{(\varphi)}\left(\underline{\bar{\varphi}}_{i}, \Delta \underline{\varphi}_{i} ; t_{i}\right) \int_{\underline{\underline{\varphi}}_{i}}^{\bar{\varphi}_{f}} \mathcal{D} \underline{\bar{\varphi}} \int_{\Delta \underline{\varphi}_{i}}^{\Delta \underline{\varphi}_{f}} \mathcal{D} \Delta \underline{\varphi} \\
& \times \exp \left\{i \int_{t_{i}}^{t_{f}} \mathrm{~d}^{4} x \underline{\mathcal{E}}(x) \cdot \Delta \underline{\varphi}(x)-\frac{1}{2} \int_{t_{i}}^{t_{f}} \mathrm{~d}^{4} x \mathrm{~d}^{4} y \sum_{a, b=1}^{N} \Delta \varphi_{a}(x) \mathcal{I}_{a b}(x, y) \Delta \varphi_{b}(y)\right\} .
\end{aligned}
$$

The term quadratic in $\Delta \underline{\varphi}$ in the argument of the exponential can be rewritten introducing auxiliary fields $\underline{\xi}=\left(\xi_{1}, \ldots, \xi_{N}\right)$,

$$
\exp \left\{-\frac{1}{2} \int_{t_{i}}^{t_{f}} \mathrm{~d}^{4} x \mathrm{~d}^{4} y \sum_{a, b=1}^{N} \Delta \varphi_{a}(x) \mathcal{I}_{a b}(x, y) \Delta \varphi_{b}(y)\right\} \equiv \int \mathcal{D} \underline{\xi} P[\underline{\xi}, \underline{\varphi}] \exp \left\{i \int_{t_{i}}^{t_{f}} \mathrm{~d}^{4} x \underline{\xi}(x) \cdot \Delta \underline{\varphi}(x)\right\}
$$

where

$$
P[\underline{\xi}, \underline{\bar{\varphi}}] \equiv \mathcal{N}[\underline{\bar{\varphi}}] \exp \left\{-\frac{1}{2} \int_{t_{i}}^{t_{f}} \mathrm{~d}^{4} x \mathrm{~d}^{4} y \sum_{a, b=1}^{N} \xi_{a}(x) \mathcal{I}_{a b}^{-1}(x, y) \xi_{b}(y)\right\}
$$

is a normalized Gaussian measure. Note that $P$ in general depends on $\underline{\bar{\varphi}}$ through $\mathcal{I}$. It is now possible to perform the functional integration over $\Delta \underline{\varphi}$ (except for the one at $t_{i}$ ) in expression (27), with the result 


$$
\rho^{(\varphi)}\left(\underline{\bar{\varphi}}_{f}, \Delta \underline{\varphi}_{f} ; t_{f}\right) \simeq \int \mathcal{D} \underline{\xi} \int \mathrm{D} \underline{\bar{\varphi}}_{i} \mathrm{D} \Delta \underline{\varphi}_{i} \rho^{(\varphi)}\left(\underline{\bar{\varphi}}_{i}, \Delta \underline{\varphi}_{i} ; t_{i}\right) \int_{\underline{\underline{\varphi}}_{i}}^{\bar{\varphi}_{f}} \mathcal{D} \underline{\bar{\varphi}} P[\underline{\xi}, \underline{\bar{\varphi}}] \delta[\underline{\mathcal{E}}(x)+\underline{\xi}(x)] .
$$

This result means that, for times $t_{i}<t<t_{f}$, the functional $\delta$ function forces the fields $\underline{\underline{\varphi}}$ to obey the classical equations of motion

$$
-\underline{\mathcal{E}}(x) \equiv-\frac{\delta S[\underline{\bar{\varphi}}]}{\delta \underline{\bar{\varphi}}(x)}-\underline{\mathcal{R}}(x)=\underline{\xi}(x) .
$$

The condition $-\delta S[\underline{\bar{\varphi}}] / \delta \underline{\bar{\varphi}}(x)=0$ is the usual classical equation of motion. The new term $\underline{\mathcal{R}}$ characterizes the interactions of the soft, classical fields $\underline{\bar{\varphi}}$ with the hard, unobserved degrees of freedom. As it will become clear in the following, a part of these interactions is to be interpreted as dissipation. The associated fluctuating noise field is represented by $\xi$ on the right-hand side of the equations of motion (31). These equations of motion are therefore Langevin-type equations. Note that in general the noise is not white, since $\mathcal{I}$ need not be proportional to $\delta\left(x_{0}-y_{0}\right)$, and can be multiplicative, due to the dependence of $\mathcal{I}$ on $\bar{\varphi}$ [8. 14. It should be mentioned at this point that multiplicative noise terms are treated slightly differently in refs. 8. 14 than in the present work. While here there is only one noise field with a $\underline{\bar{\varphi}}$-dependent variance, in [8.14] different noise fields are introduced with $\bar{\varphi}$-independent variances.

Another interesting aspect for systems with more than one field degree of freedom is that, since $\mathcal{I}$ is in general not diagonal, $\mathcal{I}_{a b}(x, y) \neq 0$ for $a \neq b$, there can be correlations between the noise terms in the equations of motion for two different field components $\bar{\varphi}_{a}$ and $\bar{\varphi}_{b}$. In the following, this general formalism will be applied to the $O(N)$ model, both in the symmetric case as well as with spontaneously broken symmetry.

\section{THE $O(N)$ MODEL IN THE SYMMETRIC CASE}

The Lagrangian of the $O(N)$ model is:

$$
\mathcal{L}(\underline{\phi})=\frac{1}{2}\left(\partial_{\mu} \underline{\phi} \cdot \partial^{\mu} \underline{\phi}-m^{2} \underline{\phi} \cdot \underline{\phi}\right)-\frac{\lambda}{N}(\underline{\phi} \cdot \underline{\phi})^{2},
$$

where $\phi=\left(\phi_{1}, \phi_{2}, \ldots, \phi_{N}\right)$ is an $N$-dimensional vector of real-valued scalar fields.

In the symmetric case, $m^{2}>0$. The vacuum state of the model is at the minimum of the potential

$$
U(\underline{\phi}) \equiv \frac{m^{2}}{2} \underline{\phi} \cdot \underline{\phi}+\frac{\lambda}{N}(\underline{\phi} \cdot \underline{\phi})^{2},
$$

which is $\underline{\phi}^{\mathrm{vac}}=0$. Decomposing the field $\underline{\phi}$ according to eqs. (9, 10), the action corresponding to interactions between soft and hard fields reads:

$$
\begin{aligned}
S_{I}[\underline{\varphi}, \underline{\Phi}]=-\frac{2 \lambda}{N} \int_{t_{i}}^{t_{f}} \mathrm{~d}^{4} x & {\left[2 \underline{\varphi}(x) \cdot \underline{\varphi}(x) \underline{\varphi}(x) \cdot \underline{\Phi}(x)+\underline{\varphi}(x) \cdot \underline{\varphi}(x) \underline{\Phi}(x) \cdot \underline{\Phi}(x)+2[\underline{\varphi}(x) \cdot \underline{\Phi}(x)]^{2}\right.} \\
& +2 \underline{\varphi}(x) \cdot \underline{\Phi}(x) \underline{\Phi}(x) \cdot \underline{\Phi}(x)] .
\end{aligned}
$$

Consequently,

$$
\begin{aligned}
&\left\langle\frac{\delta S_{I}[\overline{\underline{\varphi}}, \underline{\Phi}]}{\delta \bar{\varphi}_{a}(x)}\right\rangle_{\underline{\underline{\varphi}}}=-\frac{4 \lambda}{N}\left(2 \bar{\varphi}_{a}(x) \underline{\bar{\varphi}}(x) \cdot\langle\underline{\Phi}(x)\rangle_{\underline{\bar{\varphi}}}+\bar{\varphi}_{a}(x)\langle\underline{\Phi}(x) \cdot \underline{\Phi}(x)\rangle_{\underline{\underline{\varphi}}}+\underline{\bar{\varphi}}(x) \cdot \underline{\bar{\varphi}}(x)\left\langle\Phi_{a}(x)\right\rangle_{\underline{\underline{\varphi}}}\right. \\
&\left.+2 \underline{\bar{\varphi}}(x) \cdot\left\langle\underline{\Phi}(x) \Phi_{a}(x)\right\rangle_{\underline{\underline{\varphi}}}+\left\langle\underline{\Phi}(x) \cdot \underline{\Phi}(x) \Phi_{a}(x)\right\rangle_{\underline{\underline{\varphi}}}\right), \\
&\left\langle\frac{\delta^{2} S_{I}[\underline{\bar{\varphi}}, \underline{\Phi}]}{\delta \bar{\varphi}_{a}(x) \delta \bar{\varphi}_{b}(y)}\right\rangle_{\underline{\underline{\varphi}}}=-\frac{4 \lambda}{N}\left(2 \delta_{a b} \underline{\bar{\varphi}}(x) \cdot\langle\underline{\Phi}(x)\rangle_{\underline{\varphi}}+\delta_{a b}\langle\underline{\Phi}(x) \cdot \underline{\Phi}(x)\rangle_{\underline{\underline{\varphi}}}+2 \bar{\varphi}_{a}(x)\left\langle\Phi_{b}(x)\right\rangle_{\underline{\underline{\varphi}}}\right. \\
&\left.+2 \bar{\varphi}_{b}(x)\left\langle\Phi_{a}(x)\right\rangle_{\underline{\underline{\varphi}}}+2\left\langle\Phi_{a}(x) \Phi_{b}(x)\right\rangle_{\underline{\underline{\varphi}}}\right) \delta^{(4)}(x-y) .
\end{aligned}
$$

In the following, the averages on the right-hand side will be computed perturbatively in the coupling constant $\lambda$. The expansion in powers of $\lambda$ of the exponential in the integrand of eq. (21) reads: 


$$
\begin{aligned}
& \exp \left\{i\left(S[\underline{\Phi}]+S_{I}[\underline{\bar{\varphi}}, \underline{\Phi}]-S\left[\underline{\Phi}^{\prime}\right]-S_{I}\left[\underline{\bar{\varphi}}, \underline{\Phi}^{\prime}\right]\right)\right\} \simeq \exp \left\{i\left(S_{0}[\underline{\Phi}]-S_{0}\left[\underline{\Phi}^{\prime}\right]\right)\right\} \\
& \times\left[1-i \frac{\lambda}{N} \int_{t_{i}}^{t_{f}} \mathrm{~d}^{4} y\left(4 \underline{\bar{\varphi}}(y) \cdot \underline{\bar{\varphi}}(y) \underline{\bar{\varphi}}(y) \cdot\left[\underline{\Phi}(y)-\underline{\Phi}^{\prime}(y)\right]+2 \underline{\bar{\varphi}}(y) \cdot \underline{\bar{\varphi}}(y)\left[\underline{\Phi}(y) \cdot \underline{\Phi}(y)-\underline{\Phi}^{\prime}(y) \cdot \underline{\Phi}^{\prime}(y)\right]\right.\right. \\
& +4[\bar{\varphi}(y) \cdot \underline{\Phi}(y)]^{2}-4\left[\bar{\varphi}(y) \cdot \underline{\Phi}^{\prime}(y)\right]^{2}+4 \underline{\bar{\varphi}}(y) \cdot\left[\underline{\Phi}(y) \underline{\Phi}(y) \cdot \underline{\Phi}(y)-\underline{\Phi}^{\prime}(y) \underline{\Phi}^{\prime}(y) \cdot \underline{\Phi}^{\prime}(y)\right] \\
& \left.\left.\quad+[\underline{\Phi}(y) \cdot \underline{\Phi}(y)]^{2}-\left[\underline{\Phi}^{\prime}(y) \cdot \underline{\Phi}^{\prime}(y)\right]^{2}\right)+O\left(\lambda^{2}\right)\right],
\end{aligned}
$$

where $S_{0}[\underline{\Phi}]$ is the action for non-interacting hard fields. The quartic self-interaction term of the hard fields has been included in the perturbative treatment of $S_{I}$. Let us define an average $\langle\cdot\rangle_{0}$ in analogy to (21), where $S[\underline{\Phi}]+S_{I}[\underline{\bar{\varphi}}, \underline{\Phi}]-S\left[\underline{\Phi}^{\prime}\right]-S_{I}\left[\underline{\bar{\varphi}}, \underline{\Phi}^{\prime}\right]$ is replaced by $S_{0}[\underline{\Phi}]-S_{0}\left[\underline{\Phi}^{\prime}\right]$. If we additionally assume the initial density matrix $\hat{\rho}_{i}^{(\Phi)}$ to be of the form $\hat{\rho}_{0}^{(\Phi)} \equiv \exp \left\{-\hat{H}_{0} / T\right\} / \mathcal{Z}$, this average is then the usual thermal average in a non-interacting system at temperature $T$. Since this average involves a Gaussian measure in function space, the average (21) of an arbitrary $n$-point function $A\left(\underline{\Phi}, \underline{\Phi}^{\prime}\right)$ vanishes for odd $n$ and can be decomposed into a sum over products of $2-$ point functions for even $n$. These 2 -point functions are [19]:

$$
\begin{aligned}
\left\langle\Phi_{a}(x) \Phi_{b}(y)\right\rangle_{0} & \equiv \operatorname{Tr}\left\{\hat{\rho}_{0}^{(\Phi)} \mathrm{T}\left(\hat{\Phi}_{a}(x) \hat{\Phi}_{b}(y)\right)\right\} \equiv \delta_{a b} D_{++}(x-y) \\
& \equiv \delta_{a b}\left[D_{>}(x-y) \Theta\left(x_{0}-y_{0}\right)+D_{<}(x-y) \Theta\left(y_{0}-x_{0}\right)\right], \\
\left\langle\Phi_{a}(x) \Phi_{b}^{\prime}(y)\right\rangle_{0} & \equiv \operatorname{Tr}\left\{\hat{\rho}_{0}^{(\Phi)} \hat{\Phi}_{a}(x) \hat{\Phi}_{b}^{\prime}(y)\right\} \equiv \delta_{a b} D_{<}(x-y), \\
\left\langle\Phi_{a}^{\prime}(x) \Phi_{b}(y)\right\rangle_{0} & \equiv \operatorname{Tr}\left\{\hat{\rho}_{0}^{(\Phi)} \hat{\Phi}_{a}^{\prime}(x) \hat{\Phi}_{b}(y)\right\} \equiv \delta_{a b} D_{>}(x-y), \\
\left\langle\Phi_{a}^{\prime}(x) \Phi_{b}^{\prime}(y)\right\rangle_{0} & \equiv \operatorname{Tr}\left\{\hat{\rho}_{0}^{(\Phi)} \tilde{\mathrm{T}}\left(\hat{\Phi}_{a}^{\prime}(x) \hat{\Phi}_{b}^{\prime}(y)\right)\right\} \equiv \delta_{a b} D_{--}(x-y) \\
& \equiv \delta_{a b}\left[D_{<}(x-y) \Theta\left(x_{0}-y_{0}\right)+D_{>}(x-y) \Theta\left(y_{0}-x_{0}\right)\right],
\end{aligned}
$$

where $\mathrm{T}$ stands for time ordering, $\tilde{\mathrm{T}}$ for anti-time ordering, and where translational invariance in space-time has been assumed. The functions $D_{>}$and $D_{<}$have the Fourier representation

$$
D_{i}(t, \mathbf{x})=\int \frac{\mathrm{d}^{3} \mathbf{k}}{(2 \pi)^{3}} \Theta\left(|\mathbf{k}|-k_{c}\right) e^{i \mathbf{k} \cdot \mathbf{x}} D_{i}(t, \mathbf{k}), i=>\text { or }<,
$$

where

$$
D_{>}(t, \mathbf{k}) \equiv \frac{1}{2 E_{\mathbf{k}}}\left\{\left[1+n\left(E_{\mathbf{k}}\right)\right] e^{-i E_{\mathbf{k}} t}+n\left(E_{\mathbf{k}}\right) e^{i E_{\mathbf{k}} t}\right\} \quad, \quad D_{<}(t, \mathbf{k}) \equiv D_{>}(-t, \mathbf{k}) .
$$

Here, $n(x) \equiv\left(e^{x / T}-1\right)^{-1}$ is the Bose-Einstein distribution function, and $E_{\mathbf{k}} \equiv\left(\mathbf{k}^{2}+m^{2}\right)^{1 / 2}$.

\section{A. Interactions between soft and hard fields to first order in $\lambda$}

To determine the interaction term $\underline{\mathcal{R}}$, eq. (26b), to order $\lambda$, one needs to compute the expectation values on the right-hand side of eq. (35a) only to order $\lambda^{0}=1$, since there is already an overall factor of $\lambda$ on the right-hand side. This means that the averages $\langle\cdot\rangle_{\bar{\varphi}}$ on the right-hand side can be replaced by $\langle\cdot\rangle_{0}$. This simplification can be traced back to the fact that the functional derivatives of the influence functional in eq. (19) were rewritten in terms of averages over functional derivatives of $S_{I}$, cf. eq. (23), which itself is already of order $\lambda$. Evaluation of the averages therefore yields

$$
\begin{aligned}
\left\langle\Phi_{a}(x)\right\rangle_{\underline{\bar{\varphi}}} & \rightarrow\left\langle\Phi_{a}(x)\right\rangle_{0}=0, \\
\left\langle\Phi_{a}(x) \Phi_{b}(x)\right\rangle_{\underline{\bar{\varphi}}} & \rightarrow\left\langle\Phi_{a}(x) \Phi_{b}(x)\right\rangle_{0}=\delta_{a b} D_{++}(0), \\
\left\langle\Phi_{a}(x) \Phi_{b}^{2}(x)\right\rangle_{\underline{\underline{\varphi}}} & \rightarrow\left\langle\Phi_{a}(x) \Phi_{b}^{2}(x)\right\rangle_{0}=0 .
\end{aligned}
$$

Thus,

$$
\mathcal{R}_{a}(x)=-\frac{4(N+2) \lambda}{N} \operatorname{Re} D_{++}(0) \bar{\varphi}_{a}(x) .
$$


With eqs. (37a), (38), and (39) one derives:

$$
D_{++}(0)=\int \frac{\mathrm{d}^{3} \mathbf{k}}{(2 \pi)^{3}} \Theta\left(|\mathbf{k}|-k_{c}\right) \frac{1}{2 E_{\mathbf{k}}}\left[1+2 n\left(E_{\mathbf{k}}\right)\right] .
$$

This expression diverges, due to the vacuum contribution corresponding to the 1 in brackets. The divergence can be removed in the standard way, for instance by introducing an appropriate counterterm in the Lagrangian. In the following, it is implied that, wherever necessary, such divergences have been removed accordingly. Note that for massless particles and in the limit $k_{c} \rightarrow 0$ :

$$
\int \frac{\mathrm{d}^{3} \mathbf{k}}{(2 \pi)^{3}} \frac{1}{E_{\mathbf{k}}} n\left(E_{\mathbf{k}}\right)=\frac{T^{2}}{12} .
$$

Since the expression (42) is real-valued, one obtains for the left-hand side of the classical equation of motion for the field component $\bar{\varphi}_{a}$ :

$$
\mathcal{E}_{a}(x)=-\left[\square+m^{2}(T)+\frac{4 \lambda}{N} \underline{\bar{\varphi}}(x) \cdot \underline{\bar{\varphi}}(x)\right] \bar{\varphi}_{a}(x) .
$$

Here,

$$
m^{2}(T)=m^{2}+\frac{4(N+2) \lambda}{N} D_{++}(0)
$$

is the usual thermal mass (squared) to first order in $\lambda$. The term $\left[m^{2}(T)-m^{2}\right] \bar{\varphi}_{a}(x)$ can be graphically depicted as in Fig. 1. In this and the following graphs, external legs correspond to classical fields $\bar{\varphi}_{a}$, while internal lines correspond to propagators $D_{++}$of hard modes. The thin external leg on the left side of the vertex has no correspondence in the classical equation of motion, it corresponds to a factor $\Delta \varphi_{a}(x)$ multiplying $\mathcal{E}_{a}(x)$ in the argument of the exponential function in eq. (27). It is included here to show that the interaction in principle involves four particles.

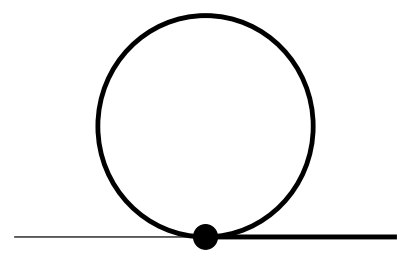

$\mathrm{X}$

FIG. 1. The order $\lambda$ contribution to the thermal mass.

The result (44) means that to first order in $\lambda$, the only effect of the presence of the hard modes (the heat bath) is a modification of the mass term in the classical equation of motion for the soft fields. There is obviously no dissipation to this order in $\lambda$. Consequently, the fluctuating field $\xi_{a}$ has to vanish, too. This is verified by explicitly computing $\mathcal{I}_{a b}(x, y)$. One first notes that the last two terms in eq. (26c) are of order $\lambda^{2}$ and thus can be neglected to first order in $\lambda$. With eq. (35b), the remaining term yields:

$$
\mathcal{I}_{a b}(x, y)=-\delta_{a b} \frac{4(N+2) \lambda}{2 N} \operatorname{Im} D_{++}(0) \delta^{(4)}(x-y),
$$

which obviously vanishes, since the expression (42) is real-valued. Therefore, to first order in $\lambda$, the introduction of a fluctuating noise field $\underline{\xi}$ via (28) is obsolete. This also implies that, to this lowest order in $\lambda$, the classical field $\bar{\varphi}(x)$ has no means to equilibrate with the background of hard modes. One has to go to the next higher order in $\lambda$ to achieve this. 


\section{B. Interactions between soft and hard fields to second order in $\lambda$}

To second order in $\lambda$, the expectation values on the right-hand side of (35) have to be evaluated to order $\lambda$. The result (neglecting terms which vanish on account of momentum conservation) is:

$$
\begin{aligned}
&\left\langle\Phi_{a}(x)\right\rangle_{\underline{\varphi}}=-i \frac{4 \lambda}{N} \int_{t_{i}}^{x_{0}} \mathrm{~d}^{4} y\left[D_{>}(x-y)-D_{<}(x-y)\right] \underline{\bar{\varphi}}(y) \cdot \underline{\bar{\varphi}}(y) \bar{\varphi}_{a}(y), \\
&\left\langle\Phi_{a}(x) \Phi_{b}(x)\right\rangle_{\underline{\underline{\varphi}}}=\delta_{a b} D_{++}(0)-i \frac{4 \lambda}{N} \int_{t_{i}}^{x_{0}} \mathrm{~d}^{4} y\left[D_{>}^{2}(x-y)-D_{<}^{2}(x-y)\right] \\
& \times\left[\delta_{a b} \underline{\underline{\varphi}}(y) \cdot \underline{\bar{\varphi}}(y)+2 \bar{\varphi}_{a}(y) \bar{\varphi}_{b}(y)+\delta_{a b}(N+2) D_{++}(0)\right], \\
&\left\langle\Phi_{a}(x) \Phi_{b}^{2}(x)\right\rangle_{\underline{\underline{\varphi}}}=-i \frac{8 \lambda}{N}\left(1+2 \delta_{a b}\right) \int_{t_{i}}^{x_{0}} \mathrm{~d}^{4} y\left[D_{>}^{3}(x-y)-D_{<}^{3}(x-y)\right] \bar{\varphi}_{a}(y) .
\end{aligned}
$$

In eq. (47d) a term was omitted which vanishes on account of momentum conservation in the expression $\int \mathrm{d}^{4} x \underline{\mathcal{E}}(x) \cdot \Delta \underline{\varphi}(x)$. The left-hand side of the equation of motion (31) is then:

$$
\mathcal{E}_{a}(x)=-\left[\square+m^{2}(T)+\frac{4 \lambda}{N} \underline{\bar{\varphi}}(x) \cdot \underline{\bar{\varphi}}(x)\right] \bar{\varphi}_{a}(x)+\left(\frac{4 \lambda}{N}\right)^{2} \sum_{i=1}^{3} \mathcal{T}_{a}^{(i)}(x)
$$

where

$$
\begin{aligned}
& \mathcal{T}_{a}^{(1)}(x) \equiv i \int_{t_{i}}^{x_{0}} \mathrm{~d}^{4} y\left[D_{>}(x-y)-D_{<}(x-y)\right]\left[\underline{\bar{\varphi}}(x) \cdot \underline{\bar{\varphi}}(x) \bar{\varphi}_{a}(y)+2 \bar{\varphi}_{a}(x) \underline{\bar{\varphi}}(x) \cdot \underline{\bar{\varphi}}(y)\right] \underline{\bar{\varphi}}(y) \cdot \underline{\bar{\varphi}}(y), \\
& \mathcal{T}_{a}^{(2)}(x) \equiv i \int_{t_{i}}^{x_{0}} \mathrm{~d}^{4} y\left[D_{>}^{2}(x-y)-D_{<}^{2}(x-y)\right]\left[(N+4) \bar{\varphi}_{a}(x) \underline{\bar{\varphi}}(y) \cdot \underline{\bar{\varphi}}(y)+4 \underline{\bar{\varphi}}(x) \cdot \underline{\bar{\varphi}}(y) \overline{\bar{\varphi}_{a}}(y)\right] \\
& \mathcal{T}_{a}^{(3)}(x) \equiv i \int_{t_{i}}^{x_{0}} \mathrm{~d}^{4} y\left[D_{>}^{3}(x-y)-D_{<}^{3}(x-y)\right] 2(N+2) \bar{\varphi}_{a}(y)
\end{aligned}
$$

and where the thermal mass $m(T)$ is given by:

$$
m^{2}(T)=m^{2}+\frac{4(N+2) \lambda}{N}\left(1-i \frac{4(N+2) \lambda}{N} \int_{t_{i}}^{x_{0}} \mathrm{~d}^{4} y\left[D_{>}^{2}(x-y)-D_{<}^{2}(x-y)\right]\right) D_{++}(0)
$$

In eqs. (48) - (50), use has been made of the fact that $D_{<}^{*}(x-y) \equiv D_{>}(x-y)$, and thus $D_{>}^{n}(x-y)-D_{<}^{n}(x-y) \equiv$ $2 i \operatorname{Im} D_{>}^{n}(x-y)$, such that all expressions in these equations are real-valued. For $N=1$ and $\lambda \rightarrow g^{2} / 4$ !, eqs. (47) - (50) reduce to the corresponding ones found in 8$]$ in $\phi^{4}$ theory. The terms $\mathcal{T}_{a}^{(i)}$ and the order $\lambda^{2}$ thermal mass correction are graphically depicted in Fig. 2. Since $-i\left[D_{>}^{n}(x-y)-D_{<}^{n}(x-y)\right]=2 \operatorname{Im} D_{>}^{n}(x-y) \equiv$ $2 \operatorname{Im} D_{++}^{n}(x-y)$ for $x_{0} \geq y_{0}$, in order to make this graphical correspondence it is implied that one has to take the imaginary part of any combination of propagators linking the space-time points $x$ and $y$ in the diagrams of Fig. 2 (as well as in the following figures). Since all external legs correspond to real-valued classical fields, however, this is equivalent to taking the imaginary part of the whole diagram. Note here the advantage of working with the functions $D$ instead of $G \equiv i D$ [8,14, which would require taking both real and imaginary parts of the diagrams, depending on the number of internal lines.

The two graphs $(\mathrm{a}, \mathrm{b})$ correspond to the two terms in the expression (49a). A sum over the external legs $b$ and $c$ is implied. Graphs $(\mathrm{c}, \mathrm{d})$ correspond to the two terms in the integrand in (49b). A sum over the external legs $b$ is implied. Figure 2 (e) corresponds to expression (49c). Finally, Fig. 2 (f) is the second order contribution to the thermal mass (times $\left.\bar{\varphi}_{a}(x)\right)$. 


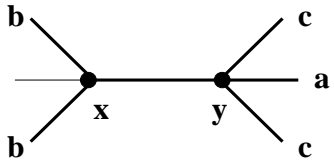

(a)

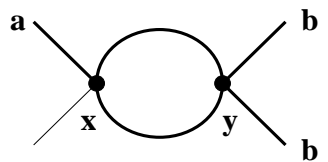

(c)

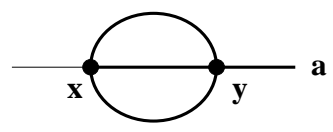

(e)

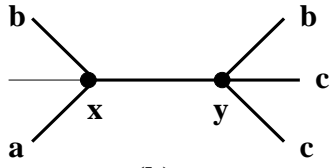

(b)

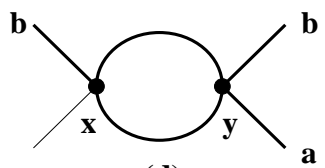

(d)

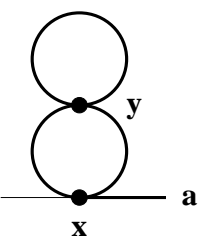

(f)

FIG. 2. (a,b) The two terms in $\mathcal{T}_{a}^{(1)}$. A sum over $b, c$ is implied. (c,d) The two terms in $\mathcal{T}_{a}^{(2)}$. A sum over $b$ is implied. (e) The term $\mathcal{T}_{a}^{(3)}$. (f) The order $\lambda^{2}$ contribution to the thermal mass.

In order to study the time evolution of the classical momentum mode functions $\bar{\varphi}_{a}(t, \mathbf{k})$, eq. (48) is Fourier transformed with respect to $\mathbf{x}$ to obtain the classical equation of motion for these modes:

$$
\begin{aligned}
& {\left[\partial_{t}^{2}+\left(E_{\mathbf{k}}^{*}\right)^{2}\right] \bar{\varphi}_{a}(t, \mathbf{k})-\left(\frac{4 \lambda}{N}\right)^{2} \sum_{i=1}^{3} \mathcal{T}_{a}^{(i)}(t, \mathbf{k})} \\
& +\frac{4 \lambda}{N} \int \frac{\mathrm{d}^{3} \mathbf{p d}^{3} \mathbf{q}}{(2 \pi)^{6}} \Theta\left(k_{c}-|\mathbf{p}|\right) \Theta\left(k_{c}-|\mathbf{q}|\right) \Theta\left(k_{c}-|\mathbf{k}-\mathbf{p}-\mathbf{q}|\right) \underline{\bar{\varphi}}(t, \mathbf{p}) \cdot \underline{\bar{\varphi}}(t, \mathbf{q}) \bar{\varphi}_{a}(t, \mathbf{k}-\mathbf{p}-\mathbf{q})=\xi_{a}(t, \mathbf{k}) .
\end{aligned}
$$

Here $E_{\mathbf{k}}^{*} \equiv\left[\mathbf{k}^{2}+m^{2}(T)\right]^{1 / 2}$. In order to see how dissipation enters in the equation of motion (51), one has to compute the three terms $\mathcal{T}_{a}^{(i)}(t, \mathbf{k}), i=1,2,3$. This calculation is rather similar to the one presented in [8] for $\phi^{4}$ theory. The details are therefore referred to Appendix A and only the main results are outlined here. From eq. (49) it is obvious that the time evolution of the classical fields prior to time $t$ influences the value of the field at time $t$. The equation of motion (51) is therefore a so-called delay-differential equation. While such integro-differential equations can in principle be solved, it is much simpler to make an Ansatz for the time evolution of the fields $\bar{\varphi}(y)$ in the terms 49 , which renders the equation of motion local in time. Such an Ansatz is the so-called linear harmonic approximation [8], cf. Appendix A. In particular, for the term $\mathcal{T}_{a}^{(3)}(t, \mathbf{k})$ one then obtains

$$
\mathcal{T}_{a}^{(3)}(t, \mathbf{k})=-2(N+2) \Theta\left(k_{c}-|\mathbf{k}|\right)\left[\mathrm{P} \int \frac{\mathrm{d} \omega}{2 \pi} \frac{\mathcal{M}_{3}(\omega, \mathbf{k})}{E_{\mathbf{k}}-\omega} \bar{\varphi}_{a}(t, \mathbf{k})+\frac{\mathcal{M}_{3}\left(E_{\mathbf{k}}, \mathbf{k}\right)}{2 E_{\mathbf{k}}} \partial_{t} \bar{\varphi}_{a}(t, \mathbf{k})\right],
$$

where $\mathrm{P}$ stands for the principal value and $\mathcal{M}_{3}(\omega, \mathbf{k})$ is the Fourier transform of $\mathcal{M}_{3}(x) \equiv D_{>}^{3}(x)-D_{<}^{3}(x)$, cf. eq. (A2 $)$. The first term represents a thermal mass correction for the field $\bar{\varphi}_{a}(t, \mathbf{k})$, while the second yields a damping term $+\eta(\mathbf{k}) \partial_{t} \bar{\varphi}_{a}(t, \mathbf{k})$ in the equation of motion (51), where the damping coefficient is:

$$
\eta(\mathbf{k}) \equiv\left(\frac{4 \lambda}{N}\right)^{2} 2(N+2) \frac{\mathcal{M}_{3}\left(E_{\mathbf{k}}, \mathbf{k}\right)}{2 E_{\mathbf{k}}} .
$$

For $N=1$ and $\lambda \rightarrow g^{2} / 4$ !, this agrees with the result (52) of [8], noting that their function $i \mathcal{M}^{(c)} \equiv g^{4} \mathcal{M}_{3} / 6$ (cf. eq. (41) of 8$]$ ). For $\mathbf{k}=0$ and $k_{c} \rightarrow 0$, the evaluation of $\mathcal{M}_{3}$ simplifies (cf. Appendix B) and yields 


$$
\eta \equiv \eta(\mathbf{0})=\left(\frac{4 \lambda}{N}\right)^{2} \frac{3(N+2) T^{2}}{32 \pi^{3} m} \operatorname{Li}_{2}\left(e^{-m / T}\right)
$$

where $\mathrm{Li}_{2}(x)$ is the dilogarithm (or Spence's integral, cf. eq. (B7)). For $\phi^{4}$ theory, i.e., $N=1$ and $\lambda \rightarrow g^{2} / 4$ ! the damping coefficient is

$$
\eta^{\phi^{4}}=\frac{g^{4} T^{2}}{128 \pi^{3} m} \operatorname{Li}_{2}\left(e^{-m / T}\right)
$$

which is twice the on-shell damping rate at zero momentum, computed to 2 -loop order in $\phi^{4}$ theory [20]. The fact that the damping coefficient is twice the damping rate was explained in detail in [8]. For the $O(N)$ model with $N=4$,

$$
\eta^{O(4)}=\frac{9 \lambda^{2} T^{2}}{16 \pi^{3} m} \operatorname{Li}_{2}\left(e^{-m / T}\right),
$$

in agreement with the result (4) of [7]. Note that in the large $-N$ limit, the damping coefficient vanishes $\sim 1 / N$.

\section{THE $O(N)$ MODEL WITH SPONTANEOUSLY BROKEN SYMMETRY}

The $O(N)$ symmetry of the Lagrangian (32) is spontaneously broken to $O(N-1)$ by taking $m^{2}<0$. Then, the potential (33) assumes the well-known "Mexican hat" shape, with the chiral circle $\left|\phi^{\mathrm{vac}}\right| \equiv$ $f_{\pi} \equiv\left(-m^{2} N / 4 \lambda\right)^{1 / 2}$ as global minimum. Adding a small explicitly symmetry breaking term $H \phi_{1}$ to the Lagrangian (32) "tilts the hat" in $\phi_{1}$-direction, such that the vacuum state is $\phi^{\mathrm{vac}}=\left(f_{\pi}, \mathbf{0}\right)$, where now $f_{\pi} \equiv\left(-m^{2} N / 4 \lambda\right)^{1 / 2} \times 2 \cos [\alpha / 3] / \sqrt{3}, \cos \alpha=(H N / 8 \lambda) /\left(-m^{2} N / 12 \lambda\right)^{3 / 2}$. The $O(N-1)$ symmetry is restored taking $H \rightarrow 0$ such that $\cos [\alpha / 3] \rightarrow \sqrt{3} / 2$.

Let us introduce new fields $\sigma \equiv \phi_{1}-f_{\pi}$ and $\boldsymbol{\pi} \equiv\left(\phi_{2}, \phi_{3}, \ldots, \phi_{N}\right)$, and corresponding masses:

$$
m_{\sigma}^{2} \equiv m^{2}+\frac{12 \lambda f_{\pi}^{2}}{N}, m_{\pi}^{2} \equiv m^{2}+\frac{4 \lambda f_{\pi}^{2}}{N}
$$

Obviously, without explicit symmetry breaking $(H=0)$, the pions are true Goldstone bosons, $m_{\pi}=0$. With these definitions the Lagrangian (32) becomes (constant terms are omitted):

$$
\mathcal{L}(\sigma, \boldsymbol{\pi})=\frac{1}{2}\left(\partial_{\mu} \sigma \partial^{\mu} \sigma-m_{\sigma}^{2} \sigma^{2}\right)+\frac{1}{2}\left(\partial_{\mu} \boldsymbol{\pi} \cdot \partial^{\mu} \boldsymbol{\pi}-m_{\pi}^{2} \boldsymbol{\pi} \cdot \boldsymbol{\pi}\right)-\frac{4 \lambda f_{\pi}}{N} \sigma\left(\sigma^{2}+\boldsymbol{\pi} \cdot \boldsymbol{\pi}\right)-\frac{\lambda}{N}\left(\sigma^{2}+\boldsymbol{\pi} \cdot \boldsymbol{\pi}\right)^{2} .
$$

Taking $N=4$, this is the Lagrangian of the linear $\sigma$ model. The parameters $m^{2}, \lambda$, and $H$ of the original Lagrangian (32) are related to the physical meson masses $m_{\sigma}=600 \mathrm{MeV}, m_{\pi}=139 \mathrm{MeV}$, and the pion decay constant $f_{\pi}=93 \mathrm{MeV}$ via $m^{2}=-\left(m_{\sigma}^{2}-3 m_{\pi}^{2}\right) / 2, \lambda=N\left(m_{\sigma}^{2}-m_{\pi}^{2}\right) /\left(8 f_{\pi}^{2}\right), H=m_{\pi}^{2} f_{\pi}$. There are two types of interaction vertices in the Lagrangian (58), a three-particle vertex proportional to $\lambda f_{\pi}$ and a four-particle vertex proportional to $\lambda$. Note that, since $f_{\pi} \sim \lambda^{-1 / 2}$, the three-particle vertex is formally of order $\lambda^{1 / 2}$.

The $\sigma$ and $\boldsymbol{\pi}$ meson fields have the Fourier representation (cf. eq. (8)

$$
\sigma(t, \mathbf{k})=\int \mathrm{d}^{3} \mathbf{x} e^{-i \mathbf{k} \cdot \mathbf{x}} \sigma(t, \mathbf{x}), \quad \boldsymbol{\pi}(t, \mathbf{k})=\int \mathrm{d}^{3} \mathbf{x} e^{-i \mathbf{k} \cdot \mathbf{x}} \boldsymbol{\pi}(t, \mathbf{x}),
$$

and are decomposed into soft and hard modes as follows:

$$
\begin{array}{rlrl}
\sigma(t, \mathbf{x})=\int \frac{\mathrm{d}^{3} \mathbf{k}}{(2 \pi)^{3}} e^{i \mathbf{k} \cdot \mathbf{x}} \sigma(t, \mathbf{k}) \Theta\left(k_{c}-|\mathbf{k}|\right) & , & \Sigma(t, \mathbf{x})=\int \frac{\mathrm{d}^{3} \mathbf{k}}{(2 \pi)^{3}} e^{i \mathbf{k} \cdot \mathbf{x}} \sigma(t, \mathbf{k}) \Theta\left(|\mathbf{k}|-k_{c}\right), \\
\boldsymbol{\pi}(t, \mathbf{x})=\int \frac{\mathrm{d}^{3} \mathbf{k}}{(2 \pi)^{3}} e^{i \mathbf{k} \cdot \mathbf{x}} \boldsymbol{\pi}(t, \mathbf{k}) \Theta\left(k_{c}-|\mathbf{k}|\right) & , \quad \boldsymbol{\Pi}(t, \mathbf{x})=\int \frac{\mathrm{d}^{3} \mathbf{k}}{(2 \pi)^{3}} e^{i \mathbf{k} \cdot \mathbf{x}} \boldsymbol{\pi}(t, \mathbf{k}) \Theta\left(|\mathbf{k}|-k_{c}\right) .
\end{array}
$$


(The use of small letters is from now on reserved for the soft fields, the hard fields are denoted by capital letters.)

The interaction between soft and hard fields is given by the action:

$$
S_{I}[\sigma, \Sigma, \boldsymbol{\pi}, \boldsymbol{\Pi}] \equiv S_{I}^{\left(\lambda f_{\pi}\right)}[\sigma, \Sigma, \boldsymbol{\pi}, \boldsymbol{\Pi}]+S_{I}^{(\lambda)}[\sigma, \Sigma, \boldsymbol{\pi}, \boldsymbol{\Pi}]
$$

where

$$
S_{I}^{\left(\lambda f_{\pi}\right)}[\sigma, \Sigma, \boldsymbol{\pi}, \boldsymbol{\Pi}]=-\frac{4 \lambda f_{\pi}}{N} \int\left(\left[3 \sigma^{2}+\boldsymbol{\pi} \cdot \boldsymbol{\pi}\right] \Sigma+2[\sigma+\Sigma] \boldsymbol{\pi} \cdot \boldsymbol{\Pi}+\sigma\left[3 \Sigma^{2}+\boldsymbol{\Pi} \cdot \boldsymbol{\Pi}\right]\right),
$$

and

$$
\begin{aligned}
S_{I}^{(\lambda)}[\sigma, \Sigma, \boldsymbol{\pi}, \boldsymbol{\Pi}]=-\frac{\lambda}{N} \int & \left(4\left[\sigma^{2}+\boldsymbol{\pi} \cdot \boldsymbol{\pi}\right][\sigma \Sigma+\boldsymbol{\pi} \cdot \boldsymbol{\Pi}]+2\left[3 \sigma^{2}+\boldsymbol{\pi} \cdot \boldsymbol{\pi}\right] \Sigma^{2}+2\left[\sigma^{2}+\boldsymbol{\pi} \cdot \boldsymbol{\pi}\right] \boldsymbol{\Pi} \cdot \boldsymbol{\Pi}\right. \\
+ & \left.4[2 \sigma \Sigma+\boldsymbol{\pi} \cdot \boldsymbol{\Pi}] \boldsymbol{\pi} \cdot \boldsymbol{\Pi}+4[\sigma \Sigma+\boldsymbol{\pi} \cdot \boldsymbol{\Pi}]\left[\Sigma^{2}+\boldsymbol{\Pi} \cdot \boldsymbol{\Pi}\right]\right) .
\end{aligned}
$$

Therefore,

$$
\begin{aligned}
& \left\langle\frac{\delta S_{I}[\bar{\sigma}, \Sigma, \overline{\boldsymbol{\pi}}, \boldsymbol{\Pi}]}{\delta \bar{\sigma}(x)}\right\rangle_{\bar{\sigma}, \bar{\pi}} \\
& =-\frac{4 \lambda f_{\pi}}{N}\left(6 \bar{\sigma}(x)\langle\Sigma(x)\rangle_{\bar{\sigma}, \bar{\pi}}+2 \overline{\boldsymbol{\pi}}(x) \cdot\langle\boldsymbol{\Pi}(x)\rangle_{\bar{\sigma}, \bar{\pi}}+3\left\langle\Sigma^{2}(x)\right\rangle_{\bar{\sigma}, \bar{\pi}}+\langle\boldsymbol{\Pi}(x) \cdot \boldsymbol{\Pi}(x)\rangle_{\bar{\sigma}, \bar{\pi}}\right) \\
& \quad-\frac{4 \lambda}{N}\left(\left[3 \bar{\sigma}^{2}(x)+\overline{\boldsymbol{\pi}}(x) \cdot \overline{\boldsymbol{\pi}}(x)\right]\langle\Sigma(x)\rangle_{\bar{\sigma}, \bar{\pi}}+2 \bar{\sigma}(x) \overline{\boldsymbol{\pi}}(x) \cdot\langle\boldsymbol{\Pi}(x)\rangle_{\bar{\sigma}, \bar{\pi}}+2 \overline{\boldsymbol{\pi}}(x) \cdot\langle\Sigma(x) \boldsymbol{\Pi}(x)\rangle_{\bar{\sigma}, \bar{\pi}}\right. \\
& \left.\quad+\bar{\sigma}(x)\left[3\left\langle\Sigma^{2}(x)\right\rangle_{\bar{\sigma}, \bar{\pi}}+\langle\boldsymbol{\Pi}(x) \cdot \boldsymbol{\Pi}(x)\rangle_{\bar{\sigma}, \bar{\pi}}\right]+\left\langle\Sigma^{3}(x)\right\rangle_{\bar{\sigma}, \bar{\pi}}+\langle\Sigma(x) \boldsymbol{\Pi}(x) \cdot \boldsymbol{\Pi}(x)\rangle_{\bar{\sigma}, \bar{\pi}}\right)
\end{aligned}
$$

while

$$
\begin{aligned}
& \left\langle\frac{\delta S_{I}[\bar{\sigma}, \Sigma, \overline{\boldsymbol{\pi}}, \boldsymbol{\Pi}]}{\delta \bar{\pi}_{a}(x)}\right\rangle_{\bar{\sigma}, \bar{\pi}}=-\frac{8 \lambda f_{\pi}}{N}\left(\bar{\pi}_{a}(x)\langle\Sigma(x)\rangle_{\bar{\sigma}, \bar{\pi}}+\bar{\sigma}(x)\left\langle\Pi_{a}(x)\right\rangle_{\bar{\sigma}, \bar{\pi}}+\left\langle\Sigma(x) \Pi_{a}(x)\right\rangle_{\bar{\sigma}, \bar{\pi}}\right) \\
& -\frac{4 \lambda}{N}\left(2 \bar{\pi}_{a}(x)\left[\bar{\sigma}(x)\langle\Sigma(x)\rangle_{\bar{\sigma}, \bar{\pi}}+\overline{\boldsymbol{\pi}}(x) \cdot\langle\boldsymbol{\Pi}(x)\rangle_{\bar{\sigma}, \bar{\pi}}\right]+\left[\bar{\sigma}^{2}(x)+\overline{\boldsymbol{\pi}}(x) \cdot \overline{\boldsymbol{\pi}}(x)\right]\left\langle\Pi_{a}(x)\right\rangle_{\bar{\sigma}, \bar{\pi}}\right. \\
& \quad+\bar{\pi}_{a}(x)\left[\left\langle\Sigma^{2}(x)\right\rangle_{\bar{\sigma}, \bar{\pi}}+\langle\boldsymbol{\Pi}(x) \cdot \boldsymbol{\Pi}(x)\rangle_{\bar{\sigma}, \bar{\pi}}\right]+2\left[\bar{\sigma}(x)\left\langle\Sigma(x) \Pi_{a}(x)\right\rangle_{\bar{\sigma}, \bar{\pi}}+\overline{\boldsymbol{\pi}}(x) \cdot\left\langle\boldsymbol{\Pi}(x) \Pi_{a}(x)\right\rangle_{\bar{\sigma}, \bar{\pi}}\right] \\
& \left.\quad+\left\langle\Sigma^{2}(x) \Pi_{a}(x)\right\rangle_{\bar{\sigma}, \bar{\pi}}+\left\langle\boldsymbol{\Pi}(x) \cdot \boldsymbol{\Pi}(x) \Pi_{a}(x)\right\rangle_{\bar{\sigma}, \bar{\pi}}\right) .
\end{aligned}
$$

\section{A. Interactions between soft and hard fields to first order in $\lambda$}

In this subsection, the expectation values $\langle\cdot\rangle_{\bar{\sigma}, \bar{\pi}}$ on the right-hand side of eqs. (64) and (65) will be evaluated in perturbation theory. Since $\lambda=N\left(m_{\sigma}^{2}-m_{\pi}^{2}\right) /\left(8 f_{\pi}^{2}\right) \simeq 20$ for $N=4$ and realistic values of the parameters $m_{\sigma}, m_{\pi}, f_{\pi}$, this is certainly not a controlled approximation scheme. Therefore, the following results have to be viewed only as the first, but necessary, step to estimate the influence of an unobserved background of hard modes in the classical equations of motion for the $O(N)$ model in the phase of spontaneously broken symmetry.

To determine the interaction terms $\mathcal{R}_{\sigma}$ and $\mathcal{R}_{\pi_{a}}$ in the classical equations of motion (31) to first order in $\lambda$, due to the overall factors of $\lambda f_{\pi} \sim \lambda^{1 / 2}$ and $\lambda$ in eqs. (64) and (65) the expectation values in the terms proportional to $\lambda f_{\pi}$ have to be computed only to order $\lambda f_{\pi}$, while those in the terms proportional to $\lambda$ have to be computed only to order 1 . More explicitly, $\langle\Sigma(x)\rangle_{\bar{\sigma}, \bar{\pi}},\left\langle\Pi_{a}(x)\right\rangle_{\bar{\sigma}, \bar{\pi}},\left\langle\Sigma^{2}(x)\right\rangle_{\bar{\sigma}, \bar{\pi}},\left\langle\Pi_{a}^{2}(x)\right\rangle_{\bar{\sigma}, \bar{\pi}}$, and $\left\langle\Sigma(x) \Pi_{a}(x)\right\rangle_{\bar{\sigma}, \bar{\pi}}$ have to be computed up to order $\lambda f_{\pi}$, while $\left\langle\Sigma^{3}(x)\right\rangle_{\bar{\sigma}, \bar{\pi}},\left\langle\Sigma^{2}(x) \Pi_{a}(x)\right\rangle_{\bar{\sigma}, \bar{\pi}},\left\langle\Sigma(x) \Pi_{a}^{2}(x)\right\rangle_{\bar{\sigma}, \bar{\pi}},\left\langle\Pi_{a}(x) \Pi_{b}(x)\right\rangle_{\bar{\sigma}, \bar{\pi}}$, and $\left\langle\Pi_{a}(x) \Pi_{b}^{2}(x)\right\rangle_{\bar{\sigma}, \bar{\pi}}$ are required to order 1. For the latter, this of course means that the average $\langle\cdot\rangle_{\bar{\sigma}, \bar{\pi}}$ can be replaced by $\langle\cdot\rangle_{0}$, and as a consequence, all expectation values vanish except for $\left\langle\Pi_{a}(x) \Pi_{b}(x)\right\rangle_{0}=\delta_{a b}\left\langle\Pi_{a}^{2}(x)\right\rangle_{0}$. For the non-vanishing expectation values one obtains: 


$$
\begin{aligned}
\langle\Sigma(x)\rangle_{\bar{\sigma}, \bar{\pi}} & =-i \frac{4 \lambda f_{\pi}}{N} \int_{t_{i}}^{x_{0}} \mathrm{~d}^{4} y\left[D_{>}^{(\sigma)}(x-y)-D_{<}^{(\sigma)}(x-y)\right]\left[3 \bar{\sigma}^{2}(y)+\bar{\pi}(y) \cdot \overline{\boldsymbol{\pi}}(y)\right], \\
\left\langle\Pi_{a}(x)\right\rangle_{\bar{\sigma}, \bar{\pi}} & =-i \frac{4 \lambda f_{\pi}}{N} \int_{t_{i}}^{x_{0}} \mathrm{~d}^{4} y\left[D_{>}^{(\pi)}(x-y)-D_{<}^{(\pi)}(x-y)\right] 2 \bar{\sigma}(y) \bar{\pi}_{a}(y), \\
\left\langle\Sigma^{2}(x)\right\rangle_{\bar{\sigma}, \bar{\pi}} & =D_{++}^{(\sigma)}(0)-i \frac{4 \lambda f_{\pi}}{N} \int_{t_{i}}^{x_{0}} \mathrm{~d}^{4} y\left(\left[D_{>}^{(\sigma)}(x-y)\right]^{2}-\left[D_{<}^{(\sigma)}(x-y)\right]^{2}\right) 6 \bar{\sigma}(y), \\
\left\langle\Pi_{a}^{2}(x)\right\rangle_{\bar{\sigma}, \bar{\pi}} & =D_{++}^{(\pi)}(0)-i \frac{4 \lambda f_{\pi}}{N} \int_{t_{i}}^{x_{0}} \mathrm{~d}^{4} y\left(\left[D_{>}^{(\pi)}(x-y)\right]^{2}-\left[D_{<}^{(\pi)}(x-y)\right]^{2}\right) 2 \bar{\sigma}(y),
\end{aligned}
$$

and

$$
\left\langle\Sigma(x) \Pi_{a}(x)\right\rangle_{\bar{\sigma}, \bar{\pi}}=-i \frac{4 \lambda f_{\pi}}{N} \int_{t_{i}}^{x_{0}} \mathrm{~d}^{4} y\left[D_{>}^{(\sigma)}(x-y) D_{>}^{(\pi)}(x-y)-D_{<}^{(\sigma)}(x-y) D_{<}^{(\pi)}(x-y)\right] 2 \bar{\pi}_{a}(y) .
$$

Again, as discussed above in the symmetric case, all expressions are real-valued. For the left-hand side of the equation of motion for the classical $\sigma$ field one therefore obtains:

$$
\begin{aligned}
\mathcal{E}_{\sigma}(x) & =-\left[\square+m_{\sigma}^{2}(T)+\frac{4 \lambda}{N}\left(\bar{\sigma}^{2}(x)+\overline{\boldsymbol{\pi}}(x) \cdot \overline{\boldsymbol{\pi}}(x)\right)\right] \bar{\sigma}(x) \\
& -\frac{4 \lambda f_{\pi}}{N}\left[3 \bar{\sigma}^{2}(x)+\overline{\boldsymbol{\pi}}(x) \cdot \overline{\boldsymbol{\pi}}(x)+3 D_{++}^{(\sigma)}(0)+(N-1) D_{++}^{(\pi)}(0)\right]+\left(\frac{4 \lambda f_{\pi}}{N}\right)^{2} \sum_{i=1}^{3} \mathcal{T}_{\sigma}^{(i)}(x),
\end{aligned}
$$

where

$$
\begin{gathered}
\mathcal{T}_{\sigma}^{(1)}(x)=i \int_{t_{i}}^{x_{0}} \mathrm{~d}^{4} y\left[D_{>}^{(\sigma)}(x-y)-D_{<}^{(\sigma)}(x-y)\right] 6 \bar{\sigma}(x)\left[3 \bar{\sigma}^{2}(y)+\overline{\boldsymbol{\pi}}(y) \cdot \overline{\boldsymbol{\pi}}(y)\right] \\
\mathcal{T}_{\sigma}^{(2)}(x)=i \int_{t_{i}}^{x_{0}} \mathrm{~d}^{4} y\left[D_{>}^{(\pi)}(x-y)-D_{<}^{(\pi)}(x-y)\right] 4 \overline{\boldsymbol{\pi}}(x) \cdot \overline{\boldsymbol{\pi}}(y) \bar{\sigma}(y) \\
\mathcal{T}_{\sigma}^{(3)}(x)=i \int_{t_{i}}^{x_{0}} \mathrm{~d}^{4} y\left\{18\left(\left[D_{>}^{(\sigma)}(x-y)\right]^{2}-\left[D_{<}^{(\sigma)}(x-y)\right]^{2}\right)\right. \\
\left.+2(N-1)\left(\left[D_{>}^{(\pi)}(x-y)\right]^{2}-\left[D_{<}^{(\pi)}(x-y)\right]^{2}\right)\right\} \bar{\sigma}(y)
\end{gathered}
$$

and $m_{\sigma}^{2}(T)$ is given by:

$$
m_{\sigma}^{2}(T)=m_{\sigma}^{2}+\frac{4 \lambda}{N}\left[3 D_{++}^{(\sigma)}(0)+(N-1) D_{++}^{(\pi)}(0)\right] .
$$

The terms $\mathcal{T}_{\sigma}^{(i)}$ and the thermal mass correction are graphically displayed in Fig. 3. The conventions are as before in Fig. 2. A dashed line represents a pion and a solid line a sigma. Thin solid lines do not have a correspondence in the classical equation of motion, they correspond to a factor $\Delta \sigma(x)$ multiplying $\mathcal{E}_{\sigma}(x)$ in the phase of the reduced density matrix (27). A filled dotted vertex corresponds to a four-particle vertex $\lambda$, a filled square vertex to a three-particle vertex $\lambda f_{\pi}$. Graphs (a,b) correspond to the two terms in eq. (68a), graph (c) to $(68 \mathrm{~b})$, graphs (d,e) to the two terms in (68d) and (f,g) to $\left[m_{\sigma}^{2}(T)-m_{\sigma}^{2}\right] \bar{\sigma}(x)$. 


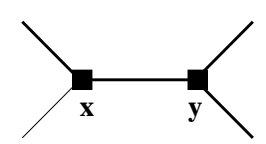

(a)

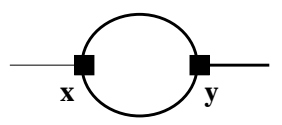

(d)

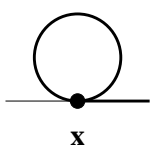

(f)

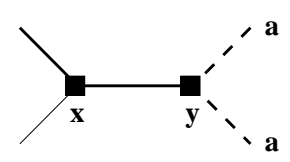

(b)

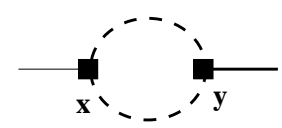

(e)

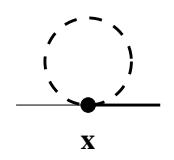

(g)

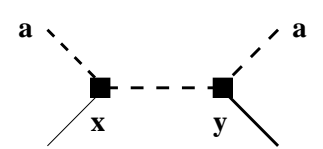

(c)

FIG. 3. (a,b) The two terms in $\mathcal{T}_{\sigma}^{(1)}$. A sum over $a$ is implied. (c) The term $\mathcal{T}_{\sigma}^{(2)}$. A sum over $a$ is implied. (d,e) The two terms in $\mathcal{T}_{\sigma}^{(3)}$. (f,g) Order $\lambda$ contributions to the thermal mass.

A Fourier transformation with respect to $\mathbf{x}$ yields the classical equation of motion for the momentum mode functions $\bar{\sigma}(t, \mathbf{k})\left(|\mathbf{k}| \leq k_{c}\right)$ :

$$
\begin{aligned}
& {\left[\partial_{t}^{2}+\left(E_{\mathbf{k}}^{(\sigma)^{*}}\right)^{2}\right] \bar{\sigma}(t, \mathbf{k})+\frac{4 \lambda f_{\pi}}{N}\left[3 D_{++}^{(\sigma)}(0)+(N-1) D_{++}^{(\pi)}(0)\right](2 \pi)^{3} \delta^{(3)}(\mathbf{k})} \\
& +\frac{4 \lambda f_{\pi}}{N} \int \frac{\mathrm{d}^{3} \mathbf{p}}{(2 \pi)^{3}} \Theta\left(k_{c}-|\mathbf{p}|\right) \Theta\left(k_{c}-|\mathbf{k}-\mathbf{p}|\right)[3 \bar{\sigma}(t, \mathbf{p}) \bar{\sigma}(t, \mathbf{k}-\mathbf{p})+\overline{\boldsymbol{\pi}}(t, \mathbf{p}) \cdot \overline{\boldsymbol{\pi}}(t, \mathbf{k}-\mathbf{p})] \\
& +\frac{4 \lambda}{N} \int \frac{\mathrm{d}^{3} \mathbf{p} \mathrm{d}^{3} \mathbf{q}}{(2 \pi)^{6}} \Theta\left(k_{c}-|\mathbf{p}|\right) \Theta\left(k_{c}-|\mathbf{q}|\right) \Theta\left(k_{c}-|\mathbf{k}-\mathbf{p}-\mathbf{q}|\right)[\bar{\sigma}(t, \mathbf{p}) \bar{\sigma}(t, \mathbf{q})+\overline{\boldsymbol{\pi}}(t, \mathbf{p}) \cdot \overline{\boldsymbol{\pi}}(t, \mathbf{q})] \bar{\sigma}(t, \mathbf{k}-\mathbf{p}-\mathbf{q}) \\
& -\left(\frac{4 \lambda f_{\pi}}{N}\right)^{2} \sum_{i=1}^{3} \mathcal{T}_{\sigma}^{(i)}(t, \mathbf{k})=\xi_{\sigma}(t, \mathbf{k}) .
\end{aligned}
$$

Here, $E_{\mathbf{k}}^{(\sigma)^{*}} \equiv\left[\mathbf{k}^{2}+m_{\sigma}^{2}(T)\right]^{1 / 2}$. An explicit calculation of the terms $\mathcal{T}_{\sigma}^{(i)}(t, \mathbf{k})$ in the linear harmonic approximation is referred to Appendix C.

For the classical pion fields one obtains:

$$
\mathcal{E}_{\pi_{a}}(x)=-\left[\square+m_{\pi}^{2}(T)+\frac{4 \lambda}{N}\left(\bar{\sigma}^{2}(x)+\overline{\boldsymbol{\pi}}(x) \cdot \overline{\boldsymbol{\pi}}(x)+2 f_{\pi} \bar{\sigma}(x)\right)\right] \bar{\pi}_{a}(x)+\left(\frac{4 \lambda f_{\pi}}{N}\right)^{2} \sum_{i=1}^{3} \mathcal{T}_{\pi_{a}}^{(i)}(x)
$$

where

$$
\begin{aligned}
& \mathcal{T}_{\pi_{a}}^{(1)}(x)=i \int_{t_{i}}^{x_{0}} \mathrm{~d}^{4} y\left[D_{>}^{(\sigma)}(x-y)-D_{<}^{(\sigma)}(x-y)\right] 2 \bar{\pi}_{a}(x)\left[3 \bar{\sigma}^{2}(y)+\overline{\boldsymbol{\pi}}(y) \cdot \overline{\boldsymbol{\pi}}(y)\right], \\
& \mathcal{T}_{\pi_{a}}^{(2)}(x)=i \int_{t_{i}}^{x_{0}} \mathrm{~d}^{4} y\left[D_{>}^{(\pi)}(x-y)-D_{<}^{(\pi)}(x-y)\right] 4 \bar{\sigma}(x) \bar{\sigma}(y) \bar{\pi}_{a}(y), \\
& \mathcal{T}_{\pi_{a}}^{(3)}(x)=i \int_{t_{i}}^{x_{0}} \mathrm{~d}^{4} y\left[D_{>}^{(\sigma)}(x-y) D_{>}^{(\pi)}(x-y)-D_{<}^{(\sigma)}(x-y) D_{<}^{(\pi)}(x-y)\right] 4 \bar{\pi}_{a}(y),
\end{aligned}
$$

and

$$
m_{\pi}^{2}(T)=m_{\pi}^{2}+\frac{4 \lambda}{N}\left[D_{++}^{(\sigma)}(0)+(N+1) D_{++}^{(\pi)}(0)\right]
$$


At first glance, $m_{\pi}(T)$ seems to be the thermal mass of soft (classical) pionic excitations. This, however, cannot be true, since in the chiral limit $m_{\pi} \rightarrow 0$, the pions are massless Goldstone bosons, even at finite temperature [21], whereas $m_{\pi}(T)$ as given by eq. (73) is finite (cf. eq. (43)). This apparent violation of Goldstone's theorem will be resolved below.

The terms $\mathcal{T}_{\pi_{a}}^{(i)}$ and the thermal mass correction are graphically displayed in Fig. $⿴$. The notation is the same as in Fig. 3. Graphs (a,b) correspond to the two terms in eq. (72a), graph (c) to (72b), graph (d) to $(\overline{72 d})$ and $(\mathrm{e}, \mathrm{f})$ to $\left[m_{\pi}^{2}(T)-m_{\pi}^{2}\right] \bar{\pi}_{a}(x)$.

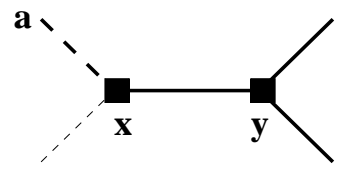

(a)

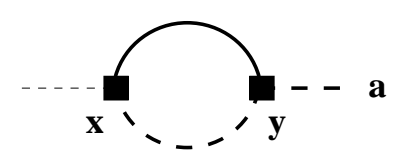

(d)

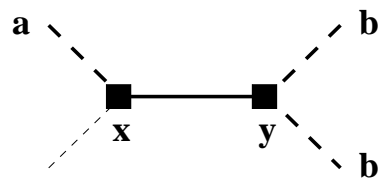

(b)

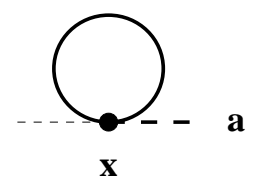

(e)

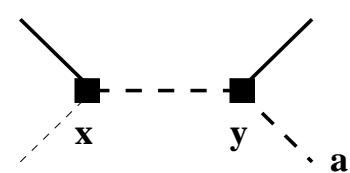

(c)

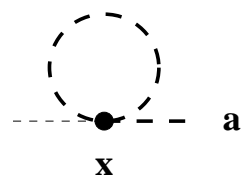

(f)

FIG. 4. (a,b) The two terms in $\mathcal{T}_{\pi_{a}}^{(1)}$. A sum over $b$ is implied. (c) The term $\mathcal{T}_{\pi_{a}}^{(2)}$. (d) The term $\mathcal{T}_{\pi_{a}}^{(3)}$. (e,f) Order $\lambda$ contributions to the thermal mass.

Fourier transforming eq. (71) with respect to $\mathbf{x}$ yields the classical equation of motion for the mode functions $\bar{\pi}_{a}(t, \mathbf{k})$ :

$$
\begin{aligned}
& {\left[\partial_{t}^{2}+\left(E_{\mathbf{k}}^{(\pi)^{*}}\right)^{2}\right] \bar{\pi}_{a}(t, \mathbf{k})+\frac{8 \lambda f_{\pi}}{N} \int \frac{\mathrm{d}^{3} \mathbf{p}}{(2 \pi)^{3}} \Theta\left(k_{c}-|\mathbf{p}|\right) \Theta\left(k_{c}-|\mathbf{k}-\mathbf{p}|\right) \bar{\sigma}(t, \mathbf{p}) \bar{\pi}_{a}(t, \mathbf{k}-\mathbf{p})} \\
& +\frac{4 \lambda}{N} \int \frac{\mathrm{d}^{3} \mathbf{p d}^{3} \mathbf{q}}{(2 \pi)^{6}} \Theta\left(k_{c}-|\mathbf{p}|\right) \Theta\left(k_{c}-|\mathbf{q}|\right) \Theta\left(k_{c}-|\mathbf{k}-\mathbf{p}-\mathbf{q}|\right)[\bar{\sigma}(t, \mathbf{p}) \bar{\sigma}(t, \mathbf{q})+\overline{\boldsymbol{\pi}}(t, \mathbf{p}) \cdot \overline{\boldsymbol{\pi}}(t, \mathbf{q})] \bar{\pi}_{a}(t, \mathbf{k}-\mathbf{p}-\mathbf{q}) \\
& -\left(\frac{4 \lambda f_{\pi}}{N}\right)^{2} \sum_{i=1}^{3} \mathcal{T}_{\pi_{a}}^{(i)}(t, \mathbf{k})=\xi_{\pi_{a}}(t, \mathbf{k})
\end{aligned}
$$

Here, $E_{\mathbf{k}}^{(\pi)^{*}} \equiv\left[\mathbf{k}^{2}+m_{\pi}^{2}(T)\right]^{1 / 2}$. An explicit calculation of the terms $\mathcal{T}_{\pi_{a}}^{(i)}(t, \mathbf{k})$ in the linear harmonic approximation is referred to Appendix D.

For the variances of the noise terms one obtains with eq. (260) up to order $\lambda$ :

$$
\begin{aligned}
& \mathcal{I}_{\sigma \sigma}(x, y)=\left(\frac{4 \lambda f_{\pi}}{N}\right)^{2}\left\{18 \bar{\sigma}(x) \bar{\sigma}(y)\left[D_{>}^{(\sigma)}(x-y)+D_{<}^{(\sigma)}(x-y)\right]\right. \\
& +2 \overline{\boldsymbol{\pi}}(x) \cdot \overline{\boldsymbol{\pi}}(y)\left[D_{>}^{(\pi)}(x-y)+D_{<}^{(\pi)}(x-y)\right] \\
& \left.+9\left(\left[D_{>}^{(\sigma)}(x-y)\right]^{2}+\left[D_{<}^{(\sigma)}(x-y)\right]^{2}\right)+(N-1)\left(\left[D_{>}^{(\pi)}(x-y)\right]^{2}+\left[D_{<}^{(\pi)}(x-y)\right]^{2}\right)\right\}, \\
& \mathcal{I}_{\sigma \pi_{a}}(x, y)=\left(\frac{4 \lambda f_{\pi}}{N}\right)^{2}\left\{6 \bar{\sigma}(x) \bar{\pi}_{a}(y)\left[D_{>}^{(\sigma)}(x-y)+D_{<}^{(\sigma)}(x-y)\right]\right. \\
& \left.+2 \bar{\pi}_{a}(x) \bar{\sigma}(y)\left[D_{>}^{(\pi)}(x-y)+D_{<}^{(\pi)}(x-y)\right]\right\}, \\
& \mathcal{I}_{\pi_{a} \pi_{b}}(x, y)=\left(\frac{4 \lambda f_{\pi}}{N}\right)^{2}\left\{2 \bar{\pi}_{a}(x) \bar{\pi}_{b}(y)\left[D_{>}^{(\sigma)}(x-y)+D_{<}^{(\sigma)}(x-y)\right]\right. \\
& +2 \delta_{a b} \bar{\sigma}(x) \bar{\sigma}(y)\left[D_{>}^{(\pi)}(x-y)+D_{<}^{(\pi)}(x-y)\right]
\end{aligned}
$$




$$
\left.+2 \delta_{a b}\left[D_{>}^{(\sigma)}(x-y) D_{>}^{(\pi)}(x-y)+D_{<}^{(\sigma)}(x-y) D_{<}^{(\pi)}(x-y)\right]\right\} .
$$

This confirms the existence of correlations between the noise fields $\xi_{\sigma}$ and $\xi_{\pi_{a}}$ as well as between $\xi_{\pi_{a}}$ and $\xi_{\pi_{b}}, a \neq b$, as mentioned at the end of section III. There also exists an obvious graphical representation for the variances. The difference to the graphs of Fig. 3 and 1 is that one thick line on the vertex at space-time point $y$ is replaced by a thin line, corresponding to the second factor $\Delta \sigma(y)$ or $\Delta \pi_{b}(y)$ in the phase in eq. (27). Also, due to $D_{>}^{n}(x)+D_{<}^{n}(x)=2 \operatorname{Re} D_{++}^{n}(x)$ one has to take the real instead of the imaginary part of the respective diagrams.

\section{B. Spatially homogeneous solutions of the classical equations of motion}

In the following, let us focus on the time evolution of the zero-momentum mode functions $\bar{\sigma}(t, \mathbf{0})$ and $\bar{\pi}_{a}(t, \mathbf{0})$. For the sake of simplicity, let us also take the limit $k_{c} \rightarrow 0$, i.e., only spatially homogeneous field configurations are considered to be classical, and let us assume $t_{i} \rightarrow-\infty, t_{f} \rightarrow+\infty$, to facilitate Fourier transformations. Then, the explicit form of the functions $\mathcal{T}_{\sigma, \pi_{a}}^{(i)}(t, \mathbf{k})$ given in Appendices $\mathrm{C}$, D shows that $\mathcal{T}_{\sigma, \pi_{a}}^{(1)}(t, \mathbf{0})=\mathcal{T}_{\sigma, \pi_{a}}^{(2)}(t, \mathbf{0})=0$, while

$$
\begin{aligned}
\mathcal{T}_{\sigma}^{(3)}(t, \mathbf{0})= & -2 \mathrm{P} \int \frac{\mathrm{d} \omega}{2 \pi} \frac{9 \mathcal{M}_{2}^{(\sigma \sigma)}(\omega, \mathbf{0})+(N-1) \mathcal{M}_{2}^{(\pi \pi)}(\omega, \mathbf{0})}{m_{\sigma}-\omega} \bar{\sigma}(t, \mathbf{0}) \\
& -2 \frac{9 \mathcal{M}_{2}^{(\sigma \sigma)}\left(m_{\sigma}, \mathbf{0}\right)+(N-1) \mathcal{M}_{2}^{(\pi \pi)}\left(m_{\sigma}, \mathbf{0}\right)}{2 m_{\sigma}} \partial_{t} \bar{\sigma}(t, \mathbf{0}),
\end{aligned}
$$

and

$$
\mathcal{T}_{\pi_{a}}^{(3)}(t, \mathbf{0})=-4 \mathrm{P} \int \frac{\mathrm{d} \omega}{2 \pi} \frac{\mathcal{M}_{2}^{(\sigma \pi)}(\omega, \mathbf{0})}{m_{\pi}-\omega} \bar{\pi}_{a}(t, \mathbf{0})-4 \frac{\mathcal{M}_{2}^{(\sigma \pi)}\left(m_{\pi}, \mathbf{0}\right)}{2 m_{\pi}} \partial_{t} \bar{\pi}_{a}(t, \mathbf{0}),
$$

where $\mathcal{M}_{2}^{(i j)}(\omega, \mathbf{k})$ is the Fourier transform of $\mathcal{M}_{2}^{(i j)}(x) \equiv D_{>}^{(i)}(x) D_{>}^{(j)}(x)-D_{<}^{(i)}(x) D_{<}^{(j)}(x), i, j=\sigma$ or $\pi$, cf. eqs. $(\mathrm{C} 1 b)$ and $(\overline{\mathrm{C} 2 b})$. Using $(2 \pi)^{3} \delta^{(3)}(\mathbf{q}) \equiv V \delta_{\mathbf{q}, \mathbf{0}}^{(3)}$, where $V$ is the total 3 -volume of the system, as well as $V \int \mathrm{d}^{3} \mathbf{p} /(2 \pi)^{3} \equiv \sum_{\mathbf{k}}$ for the momentum integrals, and defining $\bar{\sigma}(t) \equiv \bar{\sigma}(t, \mathbf{0}) / V, \overline{\boldsymbol{\pi}}(t) \equiv \overline{\boldsymbol{\pi}}(t, \mathbf{0}) / V, \xi_{\sigma}(t) \equiv$ $\xi_{\sigma}(t, \mathbf{0}) / V, \xi_{\pi_{a}}(t) \equiv \xi_{\pi_{a}}(t, \mathbf{0}) / V$, one derives from (70) the classical equation of motion for $\bar{\sigma}(t)$ :

$$
\begin{aligned}
{\left[\partial_{t}^{2}+\tilde{m}_{\sigma}^{2}(T)\right] \bar{\sigma}(t) } & +\frac{4 \lambda f_{\pi}}{N}\left[3 D_{++}^{(\sigma)}(0)+(N-1) D_{++}^{(\pi)}(0)\right]+\frac{4 \lambda f_{\pi}}{N}\left[3 \bar{\sigma}^{2}(t)+\overline{\boldsymbol{\pi}}(t) \cdot \overline{\boldsymbol{\pi}}(t)\right] \\
& +\frac{4 \lambda}{N}\left[\bar{\sigma}^{2}(t)+\overline{\boldsymbol{\pi}}(t) \cdot \overline{\boldsymbol{\pi}}(t)\right] \bar{\sigma}(t)+\eta_{\sigma} \partial_{t} \bar{\sigma}(t)=\xi_{\sigma}(t)
\end{aligned}
$$

with

$$
\begin{aligned}
\tilde{m}_{\sigma}^{2}(T) & =m_{\sigma}^{2}(T)+2\left(\frac{4 \lambda f_{\pi}}{N}\right)^{2} \mathrm{P} \int \frac{\mathrm{d} \omega}{2 \pi} \frac{9 \mathcal{M}_{2}^{(\sigma \sigma)}(\omega, \mathbf{0})+(N-1) \mathcal{M}_{2}^{(\pi \pi)}(\omega, \mathbf{0})}{m_{\sigma}-\omega} \\
& =m_{\sigma}^{2}+\frac{4 \lambda}{N}\left[3 D_{++}^{(\sigma)}(0)+(N-1) D_{++}^{(\pi)}(0)\right] \\
& +\frac{2}{\pi^{2}}\left(\frac{4 \lambda f_{\pi}}{N}\right)^{2}\left[9 \int_{m_{\sigma}}^{\infty} \mathrm{d} E \frac{\sqrt{E^{2}-m_{\sigma}^{2}}}{m_{\sigma}^{2}-4 E^{2}} \frac{1}{e^{E / T}-1}+(N-1) \int_{m_{\pi}}^{\infty} \mathrm{d} E \frac{\sqrt{E^{2}-m_{\pi}^{2}}}{m_{\sigma}^{2}-4 E^{2}} \frac{1}{e^{E / T}-1}\right],
\end{aligned}
$$

where use has been made of eqs. $(69)$ and $(\mathrm{C} 2 \mathrm{~b})$, and divergent terms have been removed by renormalization. The apparent singularity in the last integral poses no problem, since it is integrable. The dissipation coefficient is (cf. Appendix E):

$$
\begin{aligned}
\eta_{\sigma} & =\left(\frac{4 \lambda f_{\pi}}{N}\right)^{2} 2 \frac{9 \mathcal{M}_{2}^{(\sigma \sigma)}\left(m_{\sigma}, \mathbf{0}\right)+(N-1) \mathcal{M}_{2}^{(\pi \pi)}\left(m_{\sigma}, \mathbf{0}\right)}{2 m_{\sigma}} \\
& =\left(\frac{4 \lambda f_{\pi}}{N}\right)^{2} \frac{N-1}{8 \pi m_{\sigma}} \sqrt{1-\frac{4 m_{\pi}^{2}}{m_{\sigma}^{2}}} \operatorname{coth} \frac{m_{\sigma}}{4 T}
\end{aligned}
$$


The dissipation coefficient $\eta_{\sigma}$ corresponds to the imaginary part of the diagram Fig. 3 (e), where the incoming (outgoing) $\sigma$ particle is on-shell and at rest. Therefore, the dissipation occurs physically due to the decay of the $\sigma$ into two $\pi$ 's [22]. The temperature dependence of $\eta_{\sigma}$ is shown in Fig. 国. Note that even at $T=0$, the dissipation coefficient does not vanish. This is physically plausible, since even then a $\sigma$ can always decay into two $\pi$ 's. This means, however, that dissipation (and associated fluctuations) persist even in the absence of a heat bath. In that case, the fluctuations have to interpreted as quantum rather than thermal fluctuations.

For $T=0$, and for the parameters of the linear sigma model, $\eta_{\sigma}=591.45 \mathrm{MeV}$, which is on the order $m_{\sigma}$, and thus quite large. In the chiral limit, $m_{\pi} \rightarrow 0$ and at $T=0$, the dissipation coefficient $\eta_{\sigma} \rightarrow 3 m_{\sigma}^{3} /\left(32 \pi f_{\pi}^{2}\right)=745.26 \mathrm{MeV}$, which is even larger. $\eta_{\sigma}$ increases with $T$ because of Bose-Einstein enhancement of the final two-pion state at finite $T$.

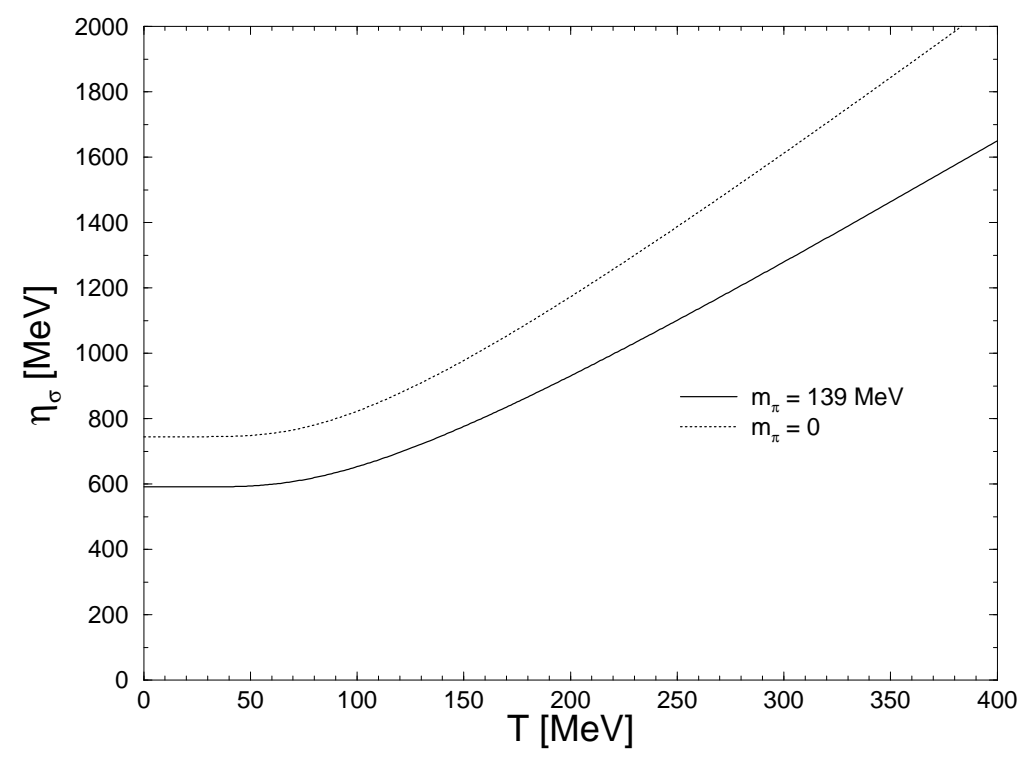

FIG. 5. The temperature dependence of the dissipation coefficient $\eta_{\sigma}$ in the case $m_{\pi}=139 \mathrm{MeV}$ (solid) and in the chiral limit $m_{\pi}=0$ (dotted).

For the variance of the noise field $\xi_{\sigma}(t)$ the so-called "white-noise" approximation is employed (cf. Appendix $\mathrm{G}$ ), which is consistent with the linear harmonic approximation (A5) that made the equation of motion for the $\sigma$ field local in time and led to the term $\eta_{\sigma} \partial_{t} \bar{\sigma}(t)$ in eq. (78). The variance of the noise field $\xi_{\sigma}(t)$ becomes

$$
\left\langle\xi_{\sigma}(t) \xi_{\sigma}\left(t^{\prime}\right)\right\rangle_{\xi}=\frac{1}{V} \delta\left(t-t^{\prime}\right) \eta_{\sigma} m_{\sigma} \operatorname{coth}\left[\frac{m_{\sigma}}{2 T}\right],
$$

where $\langle\cdot\rangle_{\xi}$ denotes the average with respect to the Gaussian measure 29). In the high-temperature limit, $\operatorname{coth}\left[m_{\sigma} / 2 T\right] \rightarrow 2 T / m_{\sigma}$, and the variance coincides with what is known from the classical fluctuationdissipation relation, which is employed in most treatments of the subject [7]. However, due to $m_{\sigma} \gg T$ for the range of temperatures of interest, this limit is not really applicable. Moreover, it would predict that the fluctuations vanish at $T=0$, while the dissipation (80) persists. This certainly contradicts the fluctuation-dissipation theorem. The more general expression (81) resolves this apparent contradiction, since $\operatorname{coth}\left[m_{\sigma} / 2 T\right] \rightarrow 1$ for $T \rightarrow 0$. Physically, the fluctuations at $T=0$ are quantum fluctuations originating from the decay of the $\sigma$ into two $\pi$ 's.

For the equation of motion of the pionic mode $\bar{\pi}_{a}(t)=\bar{\pi}_{a}(t, \mathbf{0}) / V$ one obtains from eq. (74):

$$
\left[\partial_{t}^{2}+\tilde{m}_{\pi}^{2}(T)\right] \bar{\pi}_{a}(t)+\frac{8 \lambda f_{\pi}}{N} \bar{\sigma}(t) \bar{\pi}_{a}(t)+\frac{4 \lambda}{N}\left[\bar{\sigma}^{2}(t)+\overline{\boldsymbol{\pi}}(t) \cdot \overline{\boldsymbol{\pi}}(t)\right] \bar{\pi}_{a}(t)+\eta_{\pi} \partial_{t} \bar{\pi}_{a}(t)=\xi_{\pi_{a}}(t),
$$

where

$$
\tilde{m}_{\pi}^{2}(T)=m_{\pi}^{2}(T)+4\left(\frac{4 \lambda f_{\pi}}{N}\right)^{2} \mathrm{P} \int \frac{\mathrm{d} \omega}{2 \pi} \frac{\mathcal{M}_{2}^{(\sigma \pi)}(\omega, \mathbf{0})}{m_{\pi}-\omega}=m_{\pi}^{2}+\frac{4 \lambda}{N}\left[D_{++}^{(\sigma)}(0)+(N+1) D_{++}^{(\pi)}(0)\right]
$$




$$
+\frac{2}{\pi^{2}}\left(\frac{4 \lambda f_{\pi}}{N}\right)^{2}\left[\int_{m_{\sigma}}^{\infty} \mathrm{d} E \frac{\sqrt{E^{2}-m_{\sigma}^{2}}}{m_{\sigma}^{4}-4 E^{2} m_{\pi}^{2}} \frac{m_{\sigma}^{2}}{e^{E / T}-1}-\int_{m_{\pi}}^{\infty} \mathrm{d} E \frac{\sqrt{E^{2}-m_{\pi}^{2}}}{\left(m_{\sigma}^{2}-2 m_{\pi}^{2}\right)^{2}-4 E^{2} m_{\pi}^{2}} \frac{m_{\sigma}^{2}-2 m_{\pi}^{2}}{e^{E / T}-1}\right],
$$

and (cf. Appendix F)

$$
\begin{aligned}
\eta_{\pi} & =\left(\frac{4 \lambda f_{\pi}}{N}\right)^{2} 4 \frac{\mathcal{M}_{2}^{(\sigma \pi)}\left(m_{\pi}, \mathbf{0}\right)}{2 m_{\pi}} \\
& =\left(\frac{4 \lambda f_{\pi}}{N}\right)^{2} \frac{m_{\sigma}^{2}}{4 \pi m_{\pi}^{3}} \sqrt{1-\frac{4 m_{\pi}^{2}}{m_{\sigma}^{2}}} \frac{1-\exp \left[-m_{\pi} / T\right]}{1-\exp \left[-m_{\sigma}^{2} / 2 m_{\pi} T\right]} \frac{1}{\exp \left[\left(m_{\sigma}^{2}-2 m_{\pi}^{2}\right) / 2 m_{\pi} T\right]-1} .
\end{aligned}
$$

Damping of pions arises due to the processes $\pi \pi \rightarrow \sigma$ and $\pi \sigma \rightarrow \pi$, where one $\pi$ and the $\sigma$ in the incoming channel come from the heat bath of hard modes. The temperature dependence of $\eta_{\pi}$ is shown in Fig. 6. Note that $\eta_{\pi}$ is small as compared to $\eta_{\sigma}$ in the temperature range of interest. The reason is the large $\sigma$ mass which strongly suppresses the phase space for the processes $\pi \pi \rightarrow \sigma$ and $\pi \sigma \rightarrow \pi$ (remember that one pion is at rest). Obviously, $\eta_{\pi}$ vanishes at $T=0$, because then there is no background of hard $\pi$ 's or $\sigma$ 's. It also vanishes in the chiral limit $m_{\pi} \rightarrow 0$ for all temperatures: truly massless Goldstone bosons are not damped (at least to first order in $\lambda$ and for $\mathbf{k}=0$ ). Correspondingly, the associated noise $\xi_{\pi_{a}}$ has to vanish as well. This can be explicitly seen from the expression for the variance of $\xi_{\pi_{a}}(t)$ in white-noise approximation (cf. Appendix G):

$$
\left\langle\xi_{\pi_{a}}(t) \xi_{\pi_{b}}\left(t^{\prime}\right)\right\rangle_{\xi}=\frac{1}{V} \delta_{a b} \delta\left(t-t^{\prime}\right) \eta_{\pi} m_{\pi} \operatorname{coth}\left[\frac{m_{\pi}}{2 T}\right] .
$$

Finally, it should be mentioned that for the spatially homogeneous solutions under consideration, cross correlations between different noise terms vanish (cf. Appendix G).

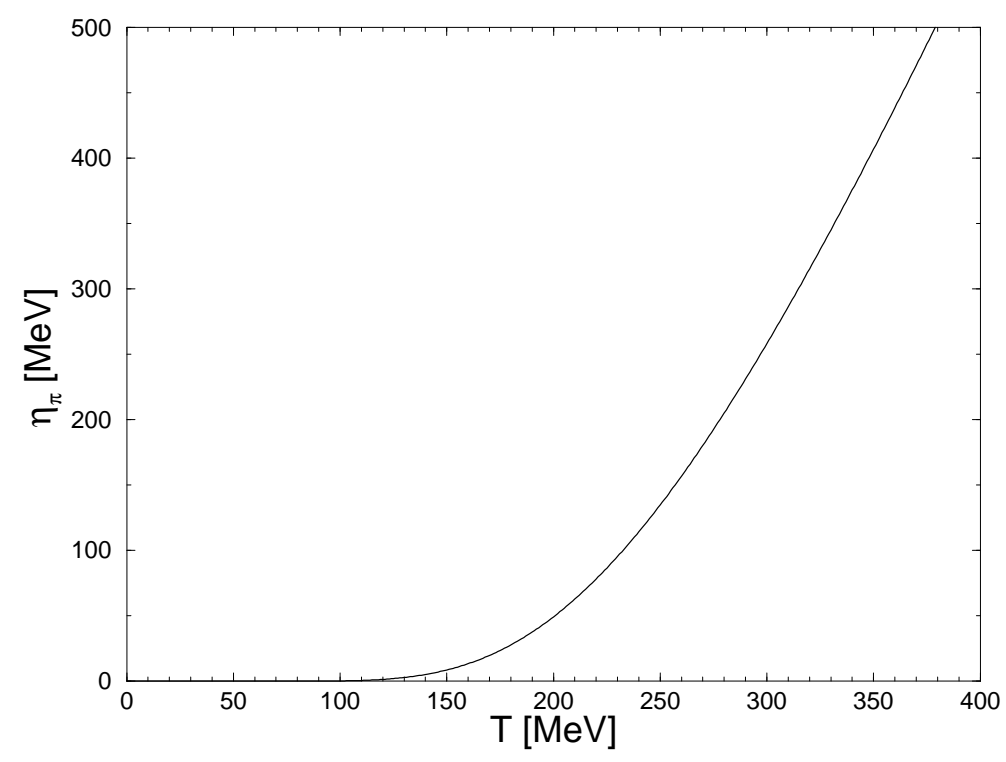

FIG. 6. The temperature dependence of the dissipation coefficient $\eta_{\pi}$.

\section{Static, spatially homogeneous solutions of the classical equations of motion}

The equations of motion (78) and (82) have the following interesting consequence. Let us consider a small perturbation $(\delta \bar{\sigma}, \delta \overline{\boldsymbol{\pi}})$ of the vacuum ground state $(\bar{\sigma}, \overline{\boldsymbol{\pi}})^{\mathrm{vac}}=0$ and let us consider the equations of motion only to lowest order in $\lambda f_{\pi} \sim \lambda^{1 / 2}$. All terms of order $\lambda$ as well as higher order terms in $\delta \bar{\sigma}$ and $\delta \overline{\boldsymbol{\pi}}$ will be neglected. To this lowest order in $\lambda$, the variances of the noise terms vanish, cf. eqs. (75), thus also $\xi_{\sigma}=\xi_{\pi_{a}} \equiv 0$, and the equations of motion have the solution 


$$
\delta \bar{\sigma}=-\frac{4 \lambda f_{\pi}}{N m_{\sigma}^{2}}\left[3 D_{++}^{(\sigma)}(0)+(N-1) D_{++}^{(\pi)}(0)\right], \quad \delta \overline{\boldsymbol{\pi}}=0 .
$$

This static solution corresponds to a constant, temperature-dependent shift of the ground state $\bar{\sigma}^{\mathrm{vac}}=$ $0 \rightarrow \bar{\sigma}^{\mathrm{vac}}=\delta \bar{\sigma}(T)$. This shift is identical to the well-known change of the vacuum ground state at finite temperatures [23]. To see this, let us consider the chiral limit $m_{\pi} \rightarrow 0$, where (for $\left.N=4\right) \lambda=m_{\sigma}^{2} /\left(2 f_{\pi}^{2}\right)$, and let us assume that $T \ll m_{\sigma}$, such that $D_{++}^{(\sigma)}(0)$ may be neglected in comparison to $D_{++}^{(\pi)}(0)$ in eq. (86). Then one obtains with eq. (43):

$$
\phi_{0}^{\mathrm{vac}} \simeq f_{\pi}\left(1-\frac{T^{2}}{8 f_{\pi}^{2}}\right)
$$

in agreement with eq. (30) of [23].

Let us now resolve the apparent contradiction of Goldstone's theorem mentioned earlier. It has to be shown that in the chiral limit $m_{\pi} \rightarrow 0$, the mass parameter for the static and homogeneous solution of eq. (82) vanishes. In the present perturbative treatment, which is accurate to order $\lambda$, one may employ the static solution (86) for the $\sigma$ and $\pi$ fields in the equation of motion (82) in all terms which are proportional to at least one power of $\lambda f_{\pi} \sim \lambda^{1 / 2}$. As shown above, the noise and fluctuation terms for the $\pi$ field vanish in the chiral limit. Thus, to order $\lambda$, one is left with the equation of motion:

$$
\hat{m}_{\pi}^{2}(T) \bar{\pi}_{a}=0
$$

where

$$
\begin{aligned}
& \hat{m}_{\pi}^{2}(T) \equiv \lim _{m_{\pi} \rightarrow 0}\left\{m_{\pi}^{2}+\frac{4 \lambda}{N}\left[D_{++}^{(\sigma)}(0)+(N+1) D_{++}^{(\pi)}(0)-\frac{8 \lambda f_{\pi}^{2}}{N m_{\sigma}^{2}}\left(3 D_{++}^{(\sigma)}(0)+(N-1) D_{++}^{(\pi)}(0)\right)\right.\right. \\
& \left.\left.+\frac{16 \lambda f_{\pi}^{2}}{N} \mathrm{P} \int \frac{\mathrm{d} \omega}{2 \pi} \frac{\mathcal{M}_{2}^{(\sigma \pi)}(\omega, \mathbf{0})}{m_{\pi}-\omega}\right]\right\} \text {. }
\end{aligned}
$$

In the limit $m_{\pi} \rightarrow 0$, the last integral can be easily calculated (cf. eq. (83)) to yield

$$
\begin{aligned}
\hat{m}_{\pi}^{2}(T)=\frac{4 \lambda}{N}\left[D_{++}^{(\sigma)}(0)+(N+1) D_{++}^{(\pi)}(0)\right. & -\frac{8 \lambda f_{\pi}^{2}}{N m_{\sigma}^{2}}\left(3 D_{++}^{(\sigma)}(0)+(N-1) D_{++}^{(\pi)}(0)\right) \\
& \left.+\frac{16 \lambda f_{\pi}^{2}}{N m_{\sigma}^{2}}\left(D_{++}^{(\sigma)}(0)-D_{++}^{(\pi)}(0)\right)\right] .
\end{aligned}
$$

In the chiral limit, the coupling constant $\lambda \rightarrow m_{\sigma}^{2} /\left(2 f_{\pi}^{2}\right)$, and all contributions to the thermal pion mass cancel, $\hat{m}_{\pi}^{2}(T) \rightarrow 0$, which completes the proof that Goldstone's theorem remains valid. In other words, the vanishing of $\hat{m}_{\pi}(T)$ implies that there exist non-trivial massless, static, homogeneous solutions to the equation of motion (88), which are, of course, nothing but the $N-1$ Goldstone bosons. Note that the above cancellation of terms is equivalent to the arguments presented in [21].

\section{NUMERICAL SOLUTIONS}

In this section, numerical solutions of the classical equations of motion (78) and (82) are presented, to assess whether DCC's can form in the presence of dissipation and fluctuation. First, note that the sign of the quantity

$$
\left[m_{\pi}^{\mathrm{eff}}(t, T)\right]^{2}=\tilde{m}_{\pi}^{2}(T)+\frac{8 \lambda f_{\pi}}{N} \bar{\sigma}(t)+\frac{4 \lambda}{N}\left[\bar{\sigma}^{2}(t)+\overline{\boldsymbol{\pi}}(t) \cdot \overline{\boldsymbol{\pi}}(t)\right]
$$

determines the time evolution of the pion fields in the absence of fluctuations or dissipation. For $\left[m_{\pi}^{\text {eff }}(t, T)\right]^{2}>0$, the pion fields simply perform oscillations with a constant amplitude, while for $\left[m_{\pi}^{\text {eff }}(t, T)\right]^{2}<0$ their amplitude grows exponentially. This exponential growth leads to large amplitude oscillations and, in turn, to a large number of pions in a given charge state (say $\pi_{3} \equiv \pi_{0}$ ). The characteristic probability to find the $\boldsymbol{\pi}$ field aligned in a certain direction in isospin space leads to a probability for the 
ratio $R$ of neutral to all pions of $P(R) \sim 1 / \sqrt{R}$. This characteristic behavior was suggested as experimental signature for the formation of DCC's [3]. Note that $\left[m_{\pi}^{\text {eff }}(t, T)\right]^{2}$ can be negative only if the second term in (91) i.e., the $\bar{\sigma}$ field, is large and negative. An explicit calculation confirms that the mass parameter $\tilde{m}_{\pi}^{2}(T)$, eq. (83), is an increasing function of $T$. Therefore, exponential growth of the pion fields (and thus formation of DCC's) is most likely (and fastest) at $T=0$. The following considerations will therefore be restricted to the case of vanishing temperature. In that case, however, there is no heat bath. Dissipation and fluctuation arise solely from the decay $\sigma \rightarrow \pi \pi$.

The equations of motion (78), (82) are solved with a standard fourth-order Runge-Kutta method. The time step width was chosen to be $\Delta t=0.002 \times 2 \pi / m_{\sigma}$. The value of the fluctuating field $\xi_{\sigma}$ is a Gaussian random number with variance $\eta_{\sigma} m_{\sigma} /(V \Delta t)$ [24]. It is chosen at the beginning of each time step and kept fixed during the Runge-Kutta step.

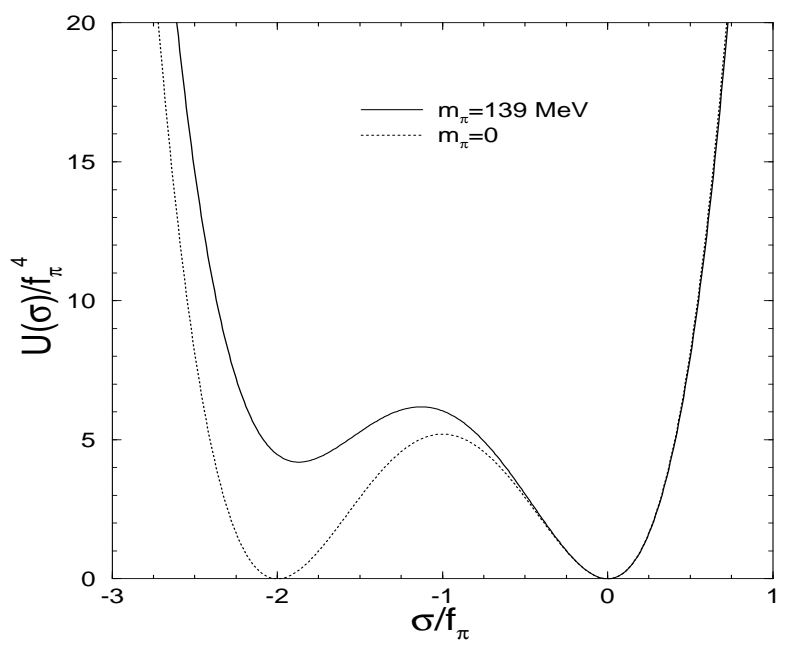

FIG. 7. The potential $U(\sigma)$ (in units of $f_{\pi}^{4}$ ) as function of $\sigma$ (in units of $f_{\pi}$ ). The solid line is for $m_{\pi}=139 \mathrm{MeV}$ and the dotted line for the chiral limit $m_{\pi}=0$.

In Fig. 7 the potential

$$
U(\sigma)=\frac{m_{\sigma}^{2}}{2} \sigma^{2}+\frac{4 \lambda f_{\pi}}{N} \sigma^{3}+\frac{\lambda}{N} \sigma^{4}
$$

is shown for $N=4, m_{\sigma}=600 \mathrm{MeV}$, and $\lambda=\left(m_{\sigma}^{2}-m_{\pi}^{2}\right) /\left(2 f_{\pi}^{2}\right)$, where $m_{\pi}=139 \mathrm{MeV}, f_{\pi}=93 \mathrm{MeV}$ (solid line). This function represents a cut through the potential energy surface of the linear sigma model at $\pi=0$, cf. eq. (58). The absolute minimum, corresponding to the ground state, is at $\sigma^{(1)}=0$. There is another local minimum at $\sigma^{(2)} \simeq-1.87 f_{\pi}$, and a local maximum at $\sigma^{(3)} \simeq-1.13 f_{\pi}$. In the chiral limit, $m_{\pi} \rightarrow 0, \sigma^{(2)} \rightarrow-2 f_{\pi}$, while $\sigma^{(3)} \rightarrow 0$ (cf. dotted line). Trajectories which are likely candidates for DCC formation obviously start at $\sigma<\sigma^{(3)}$, and small non-zero values of $\boldsymbol{\pi}$ (this is necessary because otherwise $\boldsymbol{\pi}=0$ remains a solution throughout the system's evolution).

A representative candidate is shown in Fig. 8. In part (a), the time evolution of $\bar{\sigma}$ and $\overline{\boldsymbol{\pi}}$ fields are shown for the initial conditions $\bar{\sigma}=-1.14 f_{\pi}, \bar{\pi}_{1}=0.002 f_{\pi}, \bar{\pi}_{2}=-0.001 f_{\pi} \cdot \bar{\pi}_{3}=0.001 f_{\pi}$. The derivatives of the fields are taken to be zero initially. Dissipation and fluctuation terms have also been set to zero for the solution shown in Fig. 8. One observes that the $\bar{\sigma}$ field first "rolls" towards the minimum $\sigma^{(2)}$ of the potential (92). Since this minimum is unstable in the direction of the $\overline{\boldsymbol{\pi}}$ fields, the $\bar{\sigma}$ field "rolls" on towards the absolute minimum $\sigma^{(1)}=0$. During this process the $\overline{\boldsymbol{\pi}}$ fields grow. This growth is characterized by negative values of $\left[m_{\pi}^{\text {eff }}(T)\right]^{2}$ or of $\operatorname{sgn}\left\{\left[m_{\pi}^{\text {eff }}(T)\right]^{2}\right\}\left|m_{\pi}^{\text {eff }}(T)\right|$, as shown in part (b) of Fig. \&. At this level, the evolution is conservative, and the system continues to oscillate around the ground state, since there is no way to dissipate the "potential energy" associated with the chosen initial state. This potential energy is simply converted into kinetic energy and vice versa in the course of the evolution. This also explains why the system periodically reaches the vicinity of the unstable minimum $\sigma^{(2)}$. This changes once dissipation and fluctuation is taken into account (it would also change if we solved the equations of motion in expanding 
geometries [7,25]). The large-amplitude fluctuations of the pion fields make the observation of DCC formation experimentally possible.

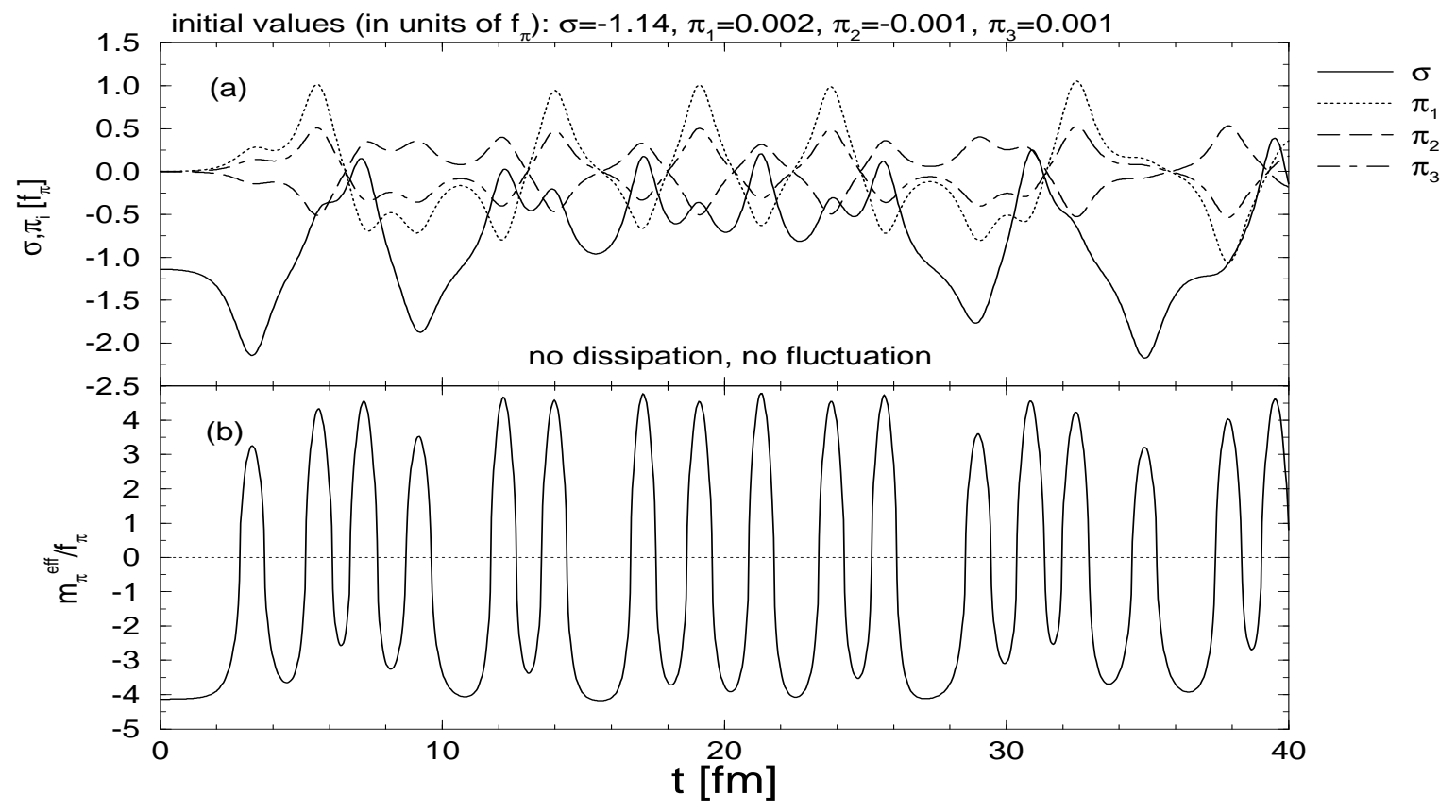

FIG. 8. (a) Classical trajectories without dissipation and fluctuations for $\bar{\sigma}$ (solid), $\bar{\pi}_{1}$ (dotted), $\bar{\pi}_{2}$ (dashed), and $\bar{\pi}_{3}$ (dash-dotted), in units of $f_{\pi}$. Initial values are $\bar{\sigma}=-1.14 f_{\pi}, \bar{\pi}_{1}=0.002 f_{\pi}, \bar{\pi}_{2}=-0.001 f_{\pi}, \bar{\pi}_{3}=0.001 f_{\pi}$. (b) The function $\operatorname{sgn}\left\{\left[m_{\pi}^{\text {eff }}\right]^{2}\right\}\left|m_{\pi}^{\text {eff }}\right|$ (in units of $f_{\pi}$ ).

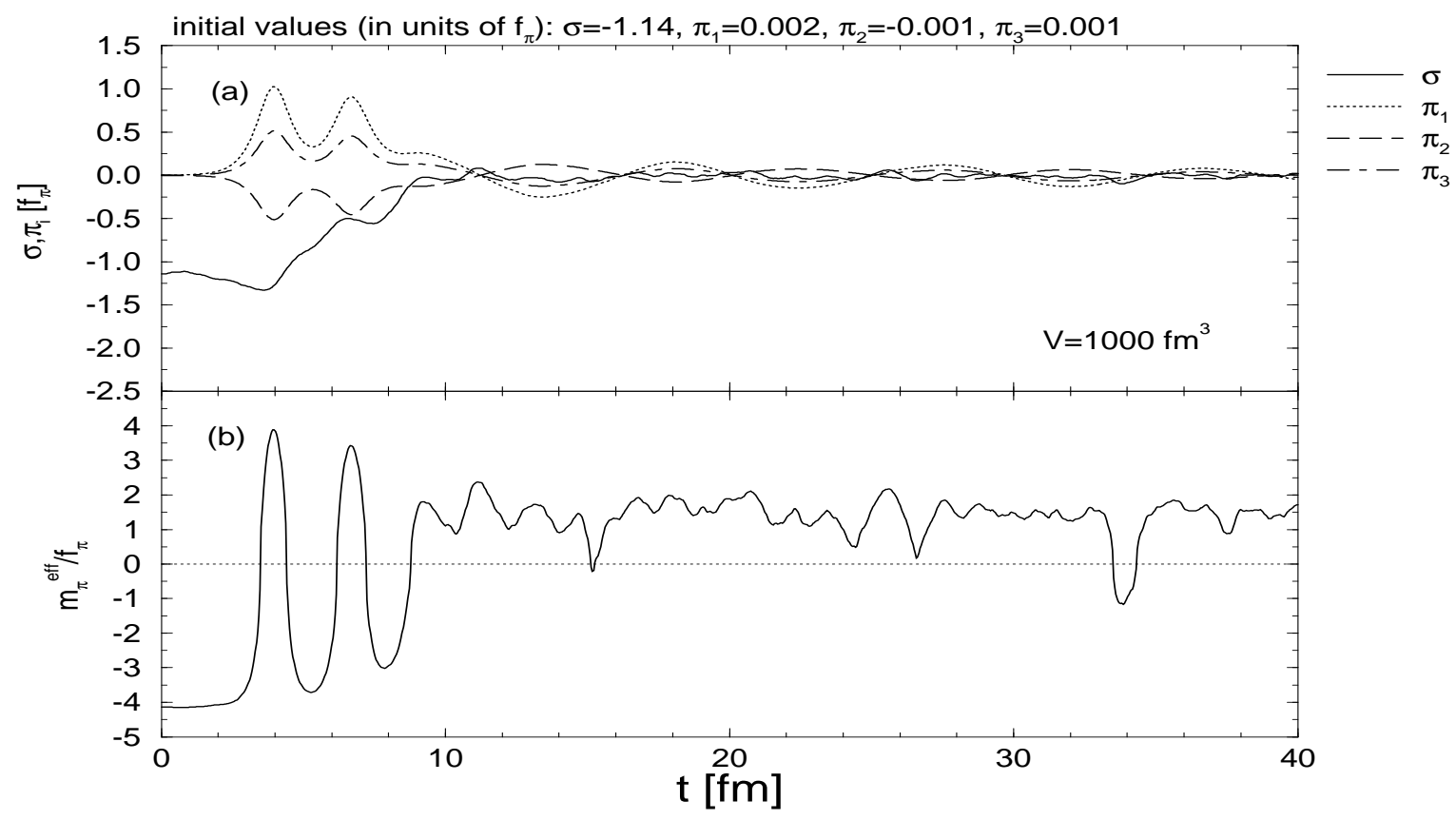

FIG. 9. (a) Trajectories with dissipation and fluctuations for $\bar{\sigma}$ (solid), $\bar{\pi}_{1}$ (dotted), $\bar{\pi}_{2}$ (dashed), and $\bar{\pi}_{3}$ (dash-dotted), in units of $f_{\pi}$. Initial values are the same as in Fig. 8 , the volume of the system is $V=1000 \mathrm{fm}^{3}$. (b) The function $\operatorname{sgn}\left\{\left[m_{\pi}^{\text {eff }}\right]^{2}\right\}\left|m_{\pi}^{\text {eff }}\right|$ (in units of $f_{\pi}$ ).

Fig. 9 (a) shows a sample trajectory including dissipation and fluctuation terms in a comparatively large 
volume $V=1000 \mathrm{fm}^{3}$ with the same initial conditions as in Fig. 8. Dissipation and fluctuations damp the oscillations of the fields, and they settle into the ground state. The time scale for this to happen is, for the sample trajectory shown here, about $10 \mathrm{fm}$. During this time, the pion fields oscillate strongly. Therefore, DCC formation would still be observable, if the system were to decouple after the first $10 \mathrm{fm}$.

This conclusion holds, however, only for the one particular trajectory shown in Fig. 9 , i.e., for one particular choice for the time evolution of the randomly fluctuating forces $\xi_{\sigma}$. For a different random sequence, the time scale for damping could be larger or smaller. In a strict sense, one would have to average over an ensemble of time evolutions for $\xi_{\sigma}$ for a given set of initial conditions for the fields [24]. The result of such an averaging is, however, predictable: on the average the fluctuations tend to cancel, and the ensemble averages of $\bar{\sigma}$ and $\overline{\boldsymbol{\pi}}$ fields are constant in time. The assumed values depend, however, on the strength of the fluctuations, i.e., since $\xi_{\sigma} \sim 1 / \sqrt{V}$, on the volume of the system. In large volumes, the fluctuations are not strong enough to "kick" the $\bar{\sigma}$ field too far out of the ground state. On the other hand, in small volumes the fluctuations may have enough strength to let the $\bar{\sigma}$ field reach the unstable minimum $\sigma^{(2)}$. Therefore, the ensemble average of the $\bar{\sigma}$ field decreases towards $-f_{\pi}$ as the volume of the system decreases (on the average, the $\bar{\sigma}$ field tends to be anywhere between the two minima $\sigma^{(1)}$ and $\sigma^{(2)}$, i.e., on the average, close to $-f_{\pi}$ ). The ensemble averages of the pion fields, however, are always zero (since the potential $U(\sigma, \boldsymbol{\pi})$ is symmetric with respect to $\boldsymbol{\pi} \rightarrow-\boldsymbol{\pi})$.

In Fig. 10 the time evolution of the fields is shown for a small volume $V=10 \mathrm{fm}^{3}$, for the same initial conditions (and the same random sequence for $\xi_{\sigma}$ ) as in the previous two figures. As discussed above, in this case fluctuations are large enough to drive the system out of the ground state and induce disorientation of the $\overline{\boldsymbol{\pi}}$ fields. One could speculate that the formation of DCC's is facilitated in smaller volumes, i.e., it should be more likely to observe them in collisions of lighter ions, or even $p p$-collisions. This could also provide an explanation for the CENTAURO events [15], where heavy ions are not likely to play any role as collision partner. To confirm this, however, a more detailed investigation in an expanding geometry, with a realistic evolution for the temperature in the collision, and including modes with finite $\mathbf{k}$ (to study domain formation) is necessary 16.

The problem with large-scale fluctuations in small volumes is, however, that one does not necessarily need to first restore chiral symmetry to observe them. In Fig. 11 a time evolution is shown in a volume $V=10 \mathrm{fm}^{3}$ for the initial condition $\bar{\sigma}=0, \bar{\pi}_{1}=0.002 f_{\pi}, \bar{\pi}_{2}=-0.001 f_{\pi}, \bar{\pi}_{3}=0.001 f_{\pi}$, i.e., the initial values of the $\overline{\boldsymbol{\pi}}$ fields are the same as before, but the $\bar{\sigma}$ field is taken to be zero. The strong fluctuations $\xi_{\sigma}$ drive the $\bar{\sigma}$ field out of the ground state, and the small initial perturbations of the pion fields are strongly enhanced over a time scale of $\simeq 10 \mathrm{fm}$ to produce large amplitude oscillations (and thus DCC formation). Note that the $\bar{\sigma}$ field is always subject to these volume-dependent fluctuations, but if one starts with the true ground state, $\bar{\sigma}=\overline{\boldsymbol{\pi}}=0$, as initial condition, the pion fields are not affected and remain zero throughout the evolution of the system. The conclusion would be that, in the presence of strong fluctuations, DCC formation is not necessarily a signal for restoration of chiral symmetry, a small perturbation of the ground state in the $\boldsymbol{\pi}$ direction seems to suffice. However, it is likely that this at first glance rather interesting phenomenon is physically identical with (and thus indistiguishable from) ordinary fluctuations in finite volumes.

Note that there is a minimum volume $V_{\text {min }}$ below which the dissipation coefficient $\eta_{\sigma}$ becomes zero. In order to have $\eta_{\sigma}>0$, the $\sigma$ at rest has to be able to decay into two pions with finite, but opposite momenta. The lowest non-zero momentum state for a particle in box volume $V=L^{3}$ is $\mathbf{k}=(\pi / L, 0,0)$. Energy conservation in the decay process requires $2 E_{\mathbf{k}}^{(\pi)} \equiv m_{\sigma}$, or $L_{\min }=2 \pi / \sqrt{m_{\sigma}^{2}-4 m_{\pi}^{2}}$, or $V_{\min } \simeq 12.68 \mathrm{fm}^{3}$, i.e., the case $V=10 \mathrm{fm}^{3}$ considered above is just on the order of the physically possible minimum volume.

\section{ACKNOWLEDGMENTS}

The author thanks C. Greiner, M. Gyulassy, U. Heinz, E. Iancu, S. Jeon, J. Kapusta, T.D. Lee, S. Leupold, L. McLerran, R. Pisarski, K. Rajagopal, and D. Son for valuable discussions, C. Greiner, B. Müller, R. Pisarski, and K. Rajagopal for a critical reading of the manuscript and suggestions, and Columbia University's Nuclear Theory Group for access to their computing facilities. 


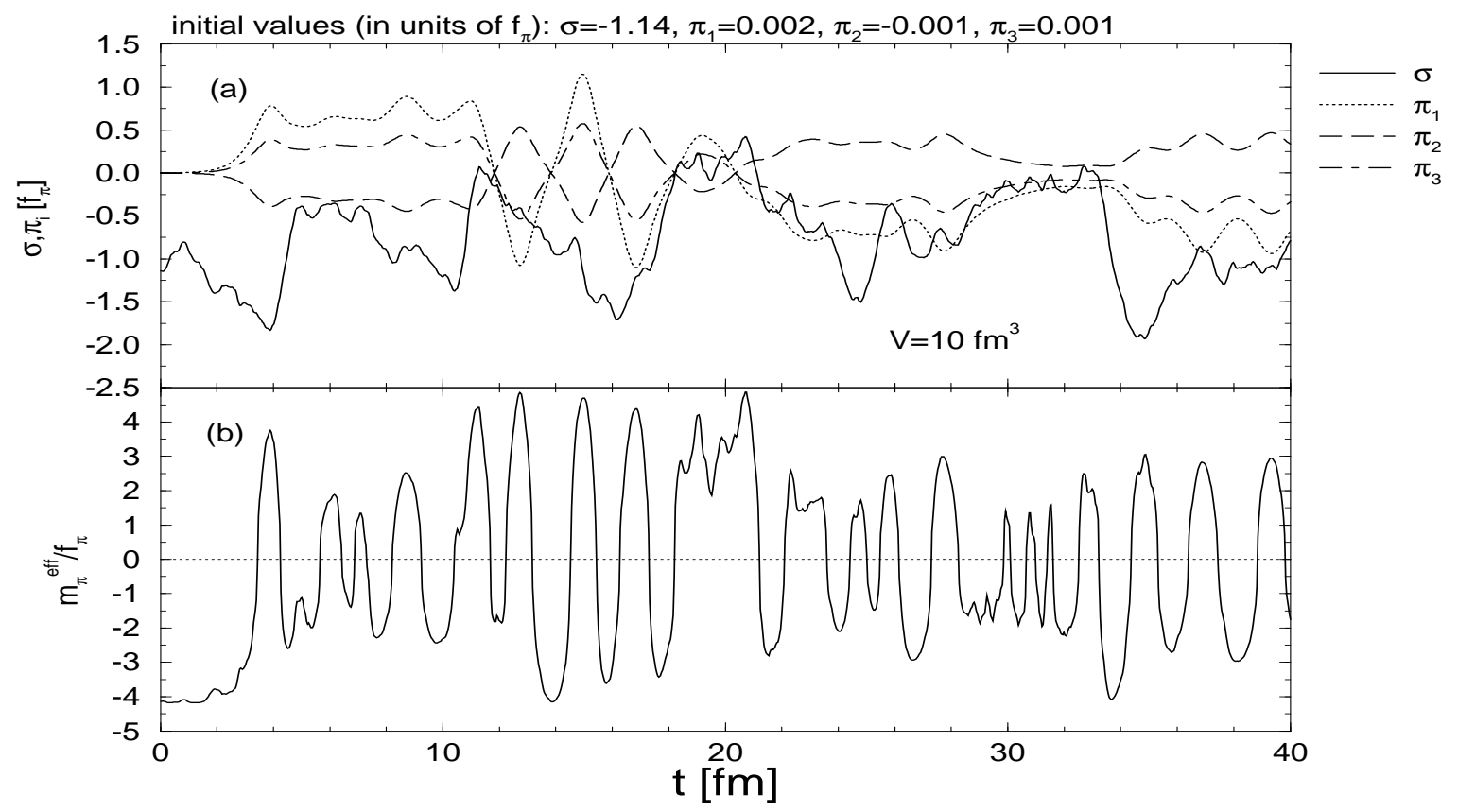

FIG. 10. (a) Trajectories with dissipation and fluctuations for $\bar{\sigma}$ (solid), $\bar{\pi}_{1}$ (dotted), $\bar{\pi}_{2}$ (dashed), and $\bar{\pi}_{3}$ (dash-dotted), in units of $f_{\pi}$. Initial values are the same as in Fig. 8, the volume of the system is $V=10 \mathrm{fm}^{3}$. (b) The function sgn $\left\{\left[m_{\pi}^{\text {eff }}\right]^{2}\right\}\left|m_{\pi}^{\text {eff }}\right|$ (in units of $f_{\pi}$ ).

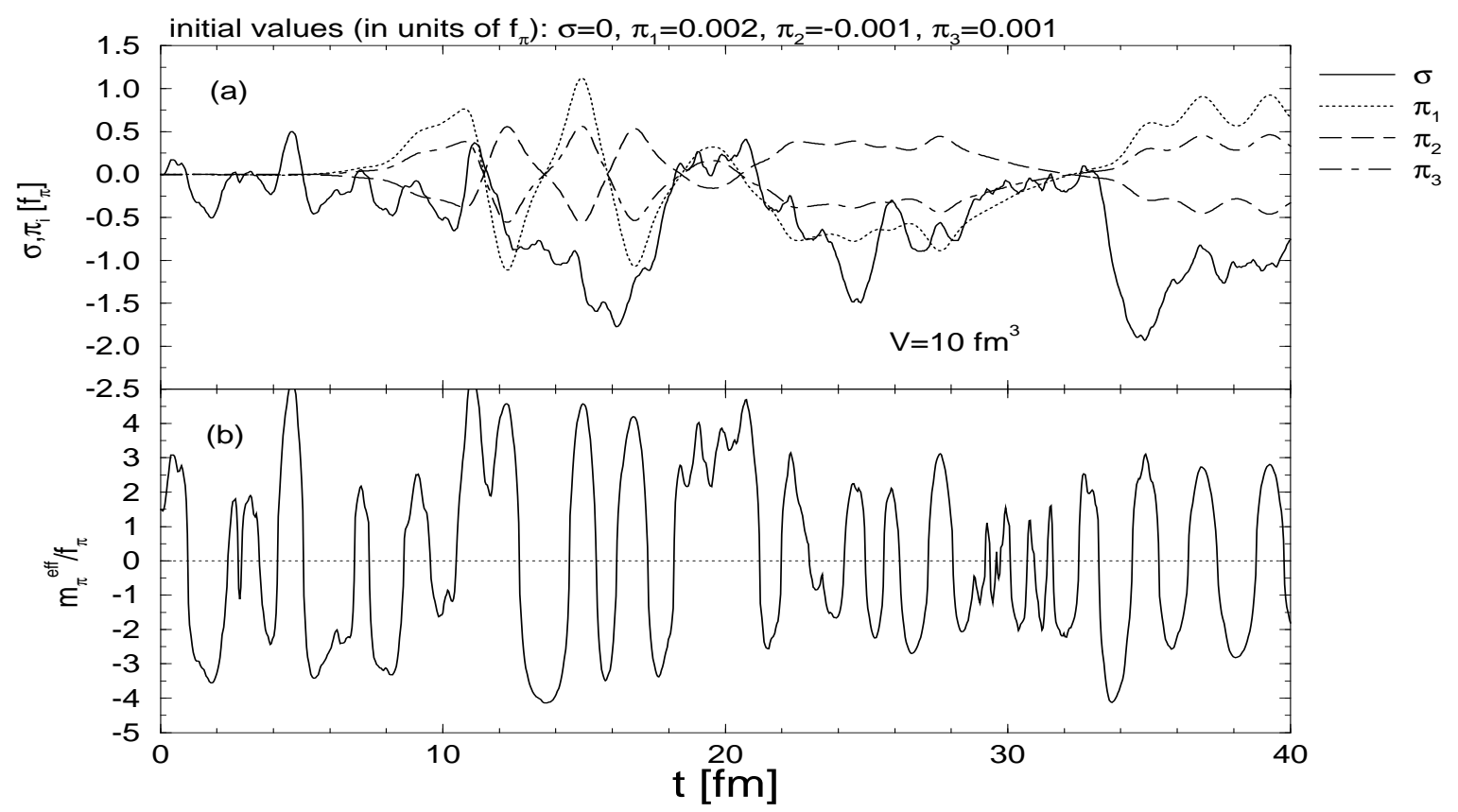

FIG. 11. (a) Trajectories with dissipation and fluctuations for $\bar{\sigma}$ (solid), $\bar{\pi}_{1}$ (dotted), $\bar{\pi}_{2}$ (dashed), and $\bar{\pi}_{3}$ (dash-dotted), in units of $f_{\pi}$. Initial values are $\bar{\sigma}=0, \bar{\pi}_{1}=0.002 f_{\pi}, \bar{\pi}_{2}=-0.001 f_{\pi}, \bar{\pi}_{3}=0.001 f_{\pi}$, the volume of the system is $V=10 \mathrm{fm}^{3}$. (b) The function $\operatorname{sgn}\left\{\left[m_{\pi}^{\text {eff }}\right]^{2}\right\}\left|m_{\pi}^{\text {eff }}\right|$ (in units of $f_{\pi}$ ). 


\section{APPENDIX A: EVALUATION OF THE INTERACTION TERMS IN THE $O(N)$ SYMMETRIC CASE}

In this Appendix, the evaluation of the interaction terms $\mathcal{T}_{a}^{(i)}(t, \mathbf{k})$ in linear harmonic approximation is presented for the $O(N)$ model in the symmetric case. Let us first define the functions

$$
\begin{aligned}
& \mathcal{M}_{1}(x)=D_{>}(x)-D_{<}(x), \\
& \mathcal{M}_{2}(x)=D_{>}^{2}(x)-D_{<}^{2}(x), \\
& \mathcal{M}_{3}(x)=D_{>}^{3}(x)-D_{<}^{3}(x)
\end{aligned}
$$

(called "memory kernels" in [8]), such that with eqs. (38), (39):

$$
\begin{aligned}
& \mathcal{M}_{1}(\omega, \mathbf{k})=\Theta\left(|\mathbf{k}|-k_{c}\right) \frac{2 \pi}{2 E_{\mathbf{k}}}\left[\delta\left(\omega-E_{\mathbf{k}}\right)-\delta\left(\omega+E_{\mathbf{k}}\right)\right], \\
& \mathcal{M}_{2}(\omega, \mathbf{k})=2 \pi \int \frac{\mathrm{d}^{3} \mathbf{p}}{(2 \pi)^{3}} \Theta\left(|\mathbf{p}|-k_{c}\right) \Theta\left(|\mathbf{k}-\mathbf{p}|-k_{c}\right) \frac{1}{4 E_{\mathbf{p}} E_{\mathbf{k}-\mathbf{p}}} \\
& \times\left\{\left(\left[1+n\left(E_{\mathbf{p}}\right)\right]\left[1+n\left(E_{\mathbf{k}-\mathbf{p}}\right)\right]-n\left(E_{\mathbf{p}}\right) n\left(E_{\mathbf{k}-\mathbf{p}}\right)\right)\left[\delta\left(\omega-E_{\mathbf{p}}-E_{\mathbf{k}-\mathbf{p}}\right)-\delta\left(\omega+E_{\mathbf{p}}+E_{\mathbf{k}-\mathbf{p}}\right)\right]\right. \\
& \left.+\left(\left[1+n\left(E_{\mathbf{p}}\right)\right] n\left(E_{\mathbf{k}-\mathbf{p}}\right)-n\left(E_{\mathbf{p}}\right)\left[1+n\left(E_{\mathbf{k}-\mathbf{p}}\right)\right]\right)\left[\delta\left(\omega-E_{\mathbf{p}}+E_{\mathbf{k}-\mathbf{p}}\right)-\delta\left(\omega+E_{\mathbf{p}}-E_{\mathbf{k}-\mathbf{p}}\right)\right]\right\}, \\
& \mathcal{M}_{3}(\omega, \mathbf{k})=2 \pi \int \frac{\mathrm{d}^{3} \mathbf{p} \mathrm{d}^{3} \mathbf{q}}{(2 \pi)^{6}} \Theta\left(|\mathbf{p}|-k_{c}\right) \Theta\left(|\mathbf{q}|-k_{c}\right) \Theta\left(|\mathbf{k}-\mathbf{p}-\mathbf{q}|-k_{c}\right) \frac{1}{8 E_{\mathbf{p}} E_{\mathbf{q}} E_{\mathbf{k}-\mathbf{p}-\mathbf{q}}} \\
& \times\left\{\left(\left[1+n\left(E_{\mathbf{p}}\right)\right]\left[1+n\left(E_{\mathbf{q}}\right)\right]\left[1+n\left(E_{\mathbf{k}-\mathbf{p}-\mathbf{q}}\right)\right]-n\left(E_{\mathbf{p}}\right) n\left(E_{\mathbf{q}}\right) n\left(E_{\mathbf{k}-\mathbf{p}-\mathbf{q}}\right)\right)\right. \\
& \times\left[\delta\left(\omega-E_{\mathbf{p}}-E_{\mathbf{q}}-E_{\mathbf{k}-\mathbf{p}-\mathbf{q}}\right)-\delta\left(\omega+E_{\mathbf{p}}+E_{\mathbf{q}}+E_{\mathbf{k}-\mathbf{p}-\mathbf{q}}\right)\right] \\
& +3\left(\left[1+n\left(E_{\mathbf{p}}\right)\right]\left[1+n\left(E_{\mathbf{q}}\right)\right] n\left(E_{\mathbf{k}-\mathbf{p}-\mathbf{q}}\right)-n\left(E_{\mathbf{p}}\right) n\left(E_{\mathbf{q}}\right)\left[1+n\left(E_{\mathbf{k}-\mathbf{p}-\mathbf{q}}\right)\right]\right) \\
& \left.\times\left[\delta\left(\omega-E_{\mathbf{p}}-E_{\mathbf{q}}+E_{\mathbf{k}-\mathbf{p}-\mathbf{q}}\right)-\delta\left(\omega+E_{\mathbf{p}}+E_{\mathbf{q}}-E_{\mathbf{k}-\mathbf{p}-\mathbf{q}}\right)\right]\right\} .
\end{aligned}
$$

Note that all functions fulfill the symmetry relation $\mathcal{M}_{i}(\omega, \mathbf{k})=-\mathcal{M}_{i}(-\omega, \mathbf{k})$. Then, eqs. (49) can be written as

$$
\begin{aligned}
& \mathcal{T}_{a}^{(1)}(x) \equiv \sum_{b}\left[\underline{\bar{\varphi}}(x) \cdot \underline{\bar{\varphi}}(x) \delta_{a b}+2 \bar{\varphi}_{a}(x) \bar{\varphi}_{b}(x)\right] \int \frac{\mathrm{d}^{3} \mathbf{k}}{(2 \pi)^{3}} e^{i \mathbf{k} \cdot \mathbf{x}} \mathcal{Y}_{b}^{(1)}\left(x_{0}, \mathbf{k}\right), \\
& \mathcal{T}_{a}^{(2)}(x) \equiv \sum_{b, c}\left[(N+4) \bar{\varphi}_{a}(x) \delta_{b c}+4 \bar{\varphi}_{b}(x) \delta_{a c}\right] \int \frac{\mathrm{d}^{3} \mathbf{k}}{(2 \pi)^{3}} e^{i \mathbf{k} \cdot \mathbf{x}} \mathcal{Y}_{b c}^{(2)}\left(x_{0}, \mathbf{k}\right) \\
& \mathcal{T}_{a}^{(3)}(x) \equiv 2(N+2) \int \frac{\mathrm{d}^{3} \mathbf{k}}{(2 \pi)^{3}} e^{i \mathbf{k} \cdot \mathbf{x}} \mathcal{Y}_{a}^{(3)}\left(x_{0}, \mathbf{k}\right)
\end{aligned}
$$

where (for $\left.t_{i} \rightarrow-\infty\right)$ :

$$
\begin{aligned}
\mathcal{Y}_{b}^{(1)}\left(x_{0}, \mathbf{k}\right) & \equiv \int \frac{\mathrm{d} \omega}{2 \pi} \mathcal{M}_{1}(\omega, \mathbf{k}) \int \frac{\mathrm{d}^{3} \mathbf{p} \mathrm{d}^{3} \mathbf{q}}{(2 \pi)^{6}} \Theta\left(k_{c}-|\mathbf{p}|\right) \Theta\left(k_{c}-|\mathbf{q}|\right) \Theta\left(k_{c}-|\mathbf{k}-\mathbf{p}-\mathbf{q}|\right) \\
& \times i \int_{0}^{\infty} \mathrm{d} \tau e^{-i \omega \tau} \bar{\varphi}_{b}\left(x_{0}-\tau, \mathbf{k}-\mathbf{p}-\mathbf{q}\right) \underline{\varphi}\left(x_{0}-\tau, \mathbf{p}\right) \cdot \bar{\varphi}\left(x_{0}-\tau, \mathbf{q}\right), \\
\mathcal{Y}_{b c}^{(2)}\left(x_{0}, \mathbf{k}\right) & \equiv \int \frac{\mathrm{d} \omega}{2 \pi} \mathcal{M}_{2}(\omega, \mathbf{k}) \int \frac{\mathrm{d}^{3} \mathbf{p}}{(2 \pi)^{3}} \Theta\left(k_{c}-|\mathbf{p}|\right) \Theta\left(k_{c}-|\mathbf{k}-\mathbf{p}|\right) \\
& \times i \int_{0}^{\infty} \mathrm{d} \tau e^{-i \omega \tau} \bar{\varphi}_{b}\left(x_{0}-\tau, \mathbf{p}\right) \bar{\varphi}_{c}\left(x_{0}-\tau, \mathbf{k}-\mathbf{p}\right), \\
\mathcal{Y}_{a}^{(3)}\left(x_{0}, \mathbf{k}\right) & \equiv \int \frac{\mathrm{d} \omega}{2 \pi} \mathcal{M}_{3}(\omega, \mathbf{k}) \Theta\left(k_{c}-|\mathbf{k}|\right) i \int_{0}^{\infty} \mathrm{d} \tau e^{-i \omega \tau} \bar{\varphi}_{a}\left(x_{0}-\tau, \mathbf{k}\right) .
\end{aligned}
$$


Further evaluation is possible by employing the so-called linear harmonic approximation [8] for the time evolution of the classical fields:

$$
\underline{\bar{\varphi}}(t-\tau, \mathbf{k}) \simeq \cos \left(E_{\mathbf{k}} \tau\right) \underline{\bar{\varphi}}(t, \mathbf{k})-\frac{\sin \left(E_{\mathbf{k}} \tau\right)}{E_{\mathbf{k}}} \partial_{t} \underline{\bar{\varphi}}(t, \mathbf{k}) .
$$

This eliminates the $\tau$ integrals over the history of the classical fields, rendering the final expressions local in time. Using the symmetry of $\mathcal{M}_{i}$ under $\omega \rightarrow-\omega$ and the relation

$$
\int_{0}^{\infty} \mathrm{d} \tau e^{i(x-\omega) \tau}=i \mathrm{P} \frac{1}{x-\omega}+\pi \delta(x-\omega),
$$

where $\mathrm{P}$ denotes the principal value, one obtains:

$$
\begin{aligned}
& \mathcal{Y}_{b}^{(1)}(t, \mathbf{k}) \simeq \int \frac{\mathrm{d}^{3} \mathbf{p} \mathrm{d}^{3} \mathbf{q}}{(2 \pi)^{6}} \Theta\left(k_{c}-|\mathbf{p}|\right) \Theta\left(k_{c}-|\mathbf{q}|\right) \Theta\left(k_{c}-|\mathbf{k}-\mathbf{p}-\mathbf{q}|\right) \\
& \times\left\{-\frac{1}{4} \mathrm{P} \int \frac{\mathrm{d} \omega}{2 \pi} \mathcal{M}_{1}(\omega, \mathbf{k})\left[\frac{1}{E_{1}+E_{2}+E_{3}-\omega}+\frac{2}{E_{1}+E_{2}-E_{3}-\omega}+\frac{1}{E_{1}-E_{2}-E_{3}-\omega}\right]\right. \\
& \times \bar{\varphi}_{b}(t, \mathbf{k}-\mathbf{p}-\mathbf{q}) \underline{\bar{\varphi}}(t, \mathbf{p}) \cdot \underline{\bar{\varphi}}(t, \mathbf{q}) \\
& +\frac{1}{4 E_{2} E_{3}} \mathrm{P} \int \frac{\mathrm{d} \omega}{2 \pi} \mathcal{M}_{1}(\omega, \mathbf{k})\left[\frac{1}{E_{1}+E_{2}+E_{3}-\omega}-\frac{2}{E_{1}+E_{2}-E_{3}-\omega}+\frac{1}{E_{1}-E_{2}-E_{3}-\omega}\right] \\
& \times \bar{\varphi}_{b}(t, \mathbf{k}-\mathbf{p}-\mathbf{q}) \partial_{t} \underline{\bar{\varphi}}(t, \mathbf{p}) \cdot \partial_{t} \underline{\bar{\varphi}}(t, \mathbf{q}) \\
& +\frac{1}{2 E_{1} E_{3}} \mathrm{P} \int \frac{\mathrm{d} \omega}{2 \pi} \mathcal{M}_{1}(\omega, \mathbf{k})\left[\frac{1}{E_{1}+E_{2}+E_{3}-\omega}-\frac{1}{E_{1}+E_{2}-E_{3}-\omega}+\frac{1}{E_{1}-E_{2}+E_{3}-\omega}\right. \\
& \left.-\frac{1}{E_{1}-E_{2}-E_{3}-\omega}\right] \partial_{t} \bar{\varphi}_{b}(t, \mathbf{k}-\mathbf{p}-\mathbf{q}) \underline{\underline{\varphi}}(t, \mathbf{p}) \cdot \partial_{t} \underline{\bar{\varphi}}(t, \mathbf{q}) \\
& -\frac{1}{4 E_{3}}\left[\mathcal{M}_{1}\left(E_{1}+E_{2}+E_{3}, \mathbf{k}\right)-\mathcal{M}_{1}\left(E_{1}+E_{2}-E_{3}, \mathbf{k}\right)+\mathcal{M}_{1}\left(E_{1}-E_{2}+E_{3}, \mathbf{k}\right)-\mathcal{M}_{1}\left(E_{1}-E_{2}-E_{3}, \mathbf{k}\right)\right] \\
& \times \bar{\varphi}_{b}(t, \mathbf{k}-\mathbf{p}-\mathbf{q}) \underline{\bar{\varphi}}(t, \mathbf{p}) \cdot \partial_{t} \underline{\bar{\varphi}}(t, \mathbf{q}) \\
& -\frac{1}{8 E_{1}}\left[\mathcal{M}_{1}\left(E_{1}+E_{2}+E_{3}, \mathbf{k}\right)+2 \mathcal{M}_{1}\left(E_{1}+E_{2}-E_{3}, \mathbf{k}\right)+\mathcal{M}_{1}\left(E_{1}-E_{2}-E_{3}, \mathbf{k}\right)\right] \\
& \times \partial_{t} \bar{\varphi}_{b}(t, \mathbf{k}-\mathbf{p}-\mathbf{q}) \underline{\bar{\varphi}}(t, \mathbf{p}) \cdot \underline{\bar{\varphi}}(t, \mathbf{q}) \\
& +\frac{1}{8 E_{1} E_{2} E_{3}}\left[\mathcal{M}_{1}\left(E_{1}+E_{2}+E_{3}, \mathbf{k}\right)-2 \mathcal{M}_{1}\left(E_{1}+E_{2}-E_{3}, \mathbf{k}\right)+\mathcal{M}_{1}\left(E_{1}-E_{2}-E_{3}, \mathbf{k}\right)\right] \\
& \left.\times \partial_{t} \bar{\varphi}_{b}(t, \mathbf{k}-\mathbf{p}-\mathbf{q}) \partial_{t} \underline{\bar{\varphi}}(t, \mathbf{p}) \cdot \partial_{t} \underline{\bar{\varphi}}(t, \mathbf{q})\right\},
\end{aligned}
$$

where $E_{1} \equiv E_{\mathbf{k}-\mathbf{p}-\mathbf{q}}, E_{2} \equiv E_{\mathbf{p}}, E_{3} \equiv E_{\mathbf{q}}$. For $N=1$, this expression reduces (up to a constant prefactor due to the difference between $\mathcal{M}^{(e)}$ and $\mathcal{M}_{1}$ ) to eq. (50) of [8]. Furthermore,

$$
\begin{aligned}
\mathcal{Y}_{b c}^{(2)}(t, \mathbf{k}) \simeq & \int \frac{\mathrm{d}^{3} \mathbf{p}}{(2 \pi)^{3}} \Theta\left(k_{c}-|\mathbf{p}|\right) \Theta\left(k_{c}-|\mathbf{k}-\mathbf{p}|\right) \\
\times & \left\{-\frac{1}{2} \mathrm{P} \int \frac{\mathrm{d} \omega}{2 \pi} \mathcal{M}_{2}(\omega, \mathbf{k})\left[\frac{1}{E_{1}+E_{2}-\omega}+\frac{1}{E_{1}-E_{2}-\omega}\right] \bar{\varphi}_{b}(t, \mathbf{p}) \bar{\varphi}_{c}(t, \mathbf{k}-\mathbf{p})\right. \\
& +\frac{1}{2 E_{1} E_{2}} \mathrm{P} \int \frac{\mathrm{d} \omega}{2 \pi} \mathcal{M}_{2}(\omega, \mathbf{k})\left[\frac{1}{E_{1}+E_{2}-\omega}-\frac{1}{E_{1}-E_{2}-\omega}\right] \partial_{t} \bar{\varphi}_{b}(t, \mathbf{p}) \partial_{t} \bar{\varphi}_{c}(t, \mathbf{k}-\mathbf{p}) \\
& -\frac{1}{4 E_{1}}\left[\mathcal{M}_{2}\left(E_{1}+E_{2}, \mathbf{k}\right)+\mathcal{M}_{2}\left(E_{1}-E_{2}, \mathbf{k}\right)\right] \bar{\varphi}_{b}(t, \mathbf{p}) \partial_{t} \bar{\varphi}_{c}(t, \mathbf{k}-\mathbf{p}) \\
& \left.-\frac{1}{4 E_{2}}\left[\mathcal{M}_{2}\left(E_{1}+E_{2}, \mathbf{k}\right)-\mathcal{M}_{2}\left(E_{1}-E_{2}, \mathbf{k}\right)\right] \partial_{t} \bar{\varphi}_{b}(t, \mathbf{p}) \bar{\varphi}_{c}(t, \mathbf{k}-\mathbf{p})\right\},
\end{aligned}
$$

where $E_{1} \equiv E_{\mathbf{k}-\mathbf{p}}, E_{2} \equiv E_{\mathbf{p}}$. For $N=1$, this is equivalent to eq. (49) of $[8]$. (There is a factor of 2 missing in front of the last term of that equation.) Finally, 


$$
\mathcal{Y}_{a}^{(3)}(t, \mathbf{k}) \simeq-\Theta\left(k_{c}-|\mathbf{k}|\right)\left[\mathrm{P} \int \frac{\mathrm{d} \omega}{2 \pi} \frac{\mathcal{M}_{3}(\omega, \mathbf{k})}{E_{\mathbf{k}}-\omega} \bar{\varphi}_{a}(t, \mathbf{k})+\frac{\mathcal{M}_{3}\left(E_{\mathbf{k}}, \mathbf{k}\right)}{2 E_{\mathbf{k}}} \partial_{t} \bar{\varphi}_{a}(t, \mathbf{k})\right]
$$

which is equivalent to eq. (48) of [8]. Then, the final expressions for the functions $\mathcal{T}_{a}^{(i)}(t, \mathbf{k})$ read:

$$
\begin{aligned}
& \mathcal{T}_{a}^{(1)}(t, \mathbf{k}) \simeq \int \frac{\mathrm{d}^{3} \mathbf{p} \mathrm{d}^{3} \mathbf{q}}{(2 \pi)^{6}} \Theta\left(k_{c}-|\mathbf{p}|\right) \Theta\left(k_{c}-|\mathbf{q}|\right) \sum_{b}\left[\underline{\underline{\varphi}}(t, \mathbf{p}) \cdot \underline{\bar{\varphi}}(t, \mathbf{q}) \delta_{a b}+2 \bar{\varphi}_{a}(t, \mathbf{p}) \bar{\varphi}_{b}(t, \mathbf{q})\right] \\
& \quad \times \mathcal{Y}_{b}^{(1)}(t, \mathbf{k}-\mathbf{p}-\mathbf{q}), \\
& \mathcal{T}_{a}^{(2)}(t, \mathbf{k}) \simeq \int \frac{\mathrm{d}^{3} \mathbf{p}}{(2 \pi)^{3}} \Theta\left(k_{c}-|\mathbf{p}|\right) \sum_{b, c}\left[(N+4) \bar{\varphi}_{a}(t, \mathbf{p}) \delta_{b c}+4 \bar{\varphi}_{b}(t, \mathbf{p}) \delta_{a c}\right] \mathcal{Y}_{b c}^{(2)}(t, \mathbf{k}-\mathbf{p}), \\
& \mathcal{T}_{a}^{(3)}(t, \mathbf{k}) \simeq 2(N+2) \mathcal{Y}_{a}^{(3)}(t, \mathbf{k}) .
\end{aligned}
$$

\section{APPENDIX B: THE DISSIPATION COEFFICIENT IN THE $O(N)$ SYMMETRIC CASE}

In order to derive the dissipation coefficient (54) one has to compute the function $\mathcal{M}_{3}(m, \mathbf{0})$. In the limit $k_{c} \rightarrow 0$ and for $\omega=m>0, \mathbf{k}=0$, one first observes that the $\delta$ functions corresponding to the decay of one particle into three and the reverse reaction (the second and third line in eq. (A2c)) have no support in this kinematic range. The only remaining contribution comes from the scattering of the particle at rest with a particle from the heat bath of hard modes (the last two lines in eq. (A2d)). Since the particles from the heat bath are supposed to be in thermal equilibrium, $n(x)=\left[e^{x / T}-1\right]^{-1}$, the reaction rates observe the detailed balance criterion, or in other words, with the energy-conserving $\delta$ functions one can rewrite the contribution from the reverse reaction in terms of a factor $e^{-\omega / T}$ times the original reaction rate. Then,

$$
\begin{aligned}
\mathcal{M}_{3}(m, \mathbf{0}) & =\frac{3 \pi}{4}(1-\exp [-m / T]) \int \frac{\mathrm{d}^{3} \mathbf{p} \mathrm{d}^{3} \mathbf{q}}{(2 \pi)^{6}} \frac{1}{E_{\mathbf{p}} E_{\mathbf{q}} E_{\mathbf{p}+\mathbf{q}}} \\
& \times\left[1+n\left(E_{\mathbf{p}}\right)\right]\left[1+n\left(E_{\mathbf{q}}\right)\right] n\left(E_{\mathbf{p}+\mathbf{q}}\right) \delta\left(E_{\mathbf{p}+\mathbf{q}}+m-E_{\mathbf{p}}-E_{\mathbf{q}}\right) .
\end{aligned}
$$

(The last $\delta$ function in the last line of eq. (A2d) has also no support for $\omega=m>0$.) This integral is most easily evaluated as follows. Let us first define $p \equiv|\mathbf{p}|, q \equiv|\mathbf{q}|, E_{x} \equiv \sqrt{x^{2}+m^{2}}$. The angular integration (involving the angle between $\mathbf{p}$ and $\mathbf{q}$ ) is substituted by an integration over $E_{\mathbf{p}+\mathbf{q}}$, with the Jacobian $\mathrm{d} E_{\mathbf{p}+\mathbf{q}} / \mathrm{d} \cos (\mathbf{p}, \mathbf{q})=p q / E_{\mathbf{p}+\mathbf{q}}$. This allows for a simple evaluation of this integral with the help of the $\delta$ function:

$$
\begin{aligned}
& \mathcal{M}_{3}(m, \mathbf{0})=\frac{3}{32 \pi^{3}}(1-\exp [-m / T]) \int_{0}^{\infty} \frac{\mathrm{d} p p}{E_{p}}\left[1+n\left(E_{p}\right)\right] \int_{0}^{\infty} \frac{\mathrm{d} q q}{E_{q}}\left[1+n\left(E_{q}\right)\right] \\
& \times n\left(E_{p}+E_{q}-m\right) \Theta\left(E_{p+q}+m-E_{p}-E_{q}\right) \Theta\left(E_{p}+E_{q}-m-E_{p-q}\right) .
\end{aligned}
$$

The $\Theta$ functions are equivalent to the constraints

$$
p q \geq\left(E_{p}-m\right)\left(E_{q}-m\right) \geq-p q .
$$

The right inequality is trivially fulfilled $\left(E_{p} \geq m\right)$, while the left is also true for all values of $p$ and $q$, since $E_{p}^{2} \leq(p+m)^{2}$. Therefore, the $\Theta$ functions do not impose additional constraints on the $p$ and $q$ integrations and can be simply omitted. Introducing the dimensionless variables $a \equiv m / T, x \equiv E_{p} / T, y \equiv E_{q} / T$, abbreviating $N(x) \equiv\left(e^{x}-1\right)^{-1}$, and noting that

$$
(1+N(y)) N(x+y-a)=N(x-a) \frac{\mathrm{d}}{\mathrm{d} y} \ln \frac{1-e^{-y}}{e^{a-x-y}-1},
$$

one arrives at

$$
\mathcal{M}_{3}(m, \mathbf{0})=\frac{3 T^{2}}{32 \pi^{3}}\left(1-e^{-a}\right) \int_{a}^{\infty} \mathrm{d} x[1+N(x)] N(x-a) \ln \frac{1-e^{-x}}{1-e^{-a}} .
$$


Introducing $t \equiv e^{-a}$, substituting $u=e^{-x}$, and then $z=(u-t) /(1-u)$, one obtains the final result

$$
\mathcal{M}_{3}(m, \mathbf{0})=\frac{3 T^{2}}{32 \pi^{3}} \operatorname{Li}_{2}\left(e^{-m / T}\right),
$$

where

$$
\operatorname{Li}_{2}(t) \equiv-\int_{0}^{1} \frac{\mathrm{d} z}{z} \ln (1-z t)
$$

is the dilogarithm, or Spence's integral.

\section{APPENDIX C: THE INTERACTION TERMS IN THE EQUATION OF MOTION FOR THE $\sigma$ FIELD}

In analogy to eqs. (A1) let us define

$$
\begin{aligned}
\mathcal{M}_{1}^{(i)}(x) & =D_{>}^{(i)}(x)-D_{<}^{(i)}(x), \\
\mathcal{M}_{2}^{(i j)}(x) & =D_{>}^{(i)}(x) D_{>}^{(j)}(x)-D_{<}^{(i)}(x) D_{<}^{(j)}(x), i, j=\sigma \text { or } \pi .
\end{aligned}
$$

The Fourier transforms are rather similar to those in eqs. (A2):

$$
\begin{aligned}
\mathcal{M}_{1}^{(i)}(\omega, \mathbf{k})= & \Theta\left(|\mathbf{k}|-k_{c}\right) \frac{2 \pi}{2 E_{\mathbf{k}}^{(i)}}\left[\delta\left(\omega-E_{\mathbf{k}}^{(i)}\right)-\delta\left(\omega+E_{\mathbf{k}}^{(i)}\right)\right], \\
\mathcal{M}_{2}^{(i j)}(\omega, \mathbf{k})=2 \pi & \int \frac{\mathrm{d}^{3} \mathbf{p}}{(2 \pi)^{3}} \Theta\left(|\mathbf{p}|-k_{c}\right) \Theta\left(|\mathbf{k}-\mathbf{p}|-k_{c}\right) \frac{1}{4 E_{\mathbf{p}}^{(i)} E_{\mathbf{k}-\mathbf{p}}^{(j)}}, \\
\times\{ & \left(\left[1+n\left(E_{\mathbf{p}}^{(i)}\right)\right]\left[1+n\left(E_{\mathbf{k}-\mathbf{p}}^{(j)}\right)\right]-n\left(E_{\mathbf{p}}^{(i)}\right) n\left(E_{\mathbf{k}-\mathbf{p}}^{(j)}\right)\right) \\
& \times\left[\delta\left(\omega-E_{\mathbf{p}}^{(i)}-E_{\mathbf{k}-\mathbf{p}}^{(j)}\right)-\delta\left(\omega+E_{\mathbf{p}}^{(i)}+E_{\mathbf{k}-\mathbf{p}}^{(j)}\right)\right] \\
+ & \left(\left[1+n\left(E_{\mathbf{p}}^{(i)}\right)\right] n\left(E_{\mathbf{k}-\mathbf{p}}^{(j)}\right)-n\left(E_{\mathbf{p}}^{(i)}\right)\left[1+n\left(E_{\mathbf{k}-\mathbf{p}}^{(j)}\right)\right]\right) \\
& \left.\times\left[\delta\left(\omega-E_{\mathbf{p}}^{(i)}+E_{\mathbf{k}-\mathbf{p}}^{(j)}\right)-\delta\left(\omega+E_{\mathbf{p}}^{(i)}-E_{\mathbf{k}-\mathbf{p}}^{(j)}\right)\right]\right\} .
\end{aligned}
$$

Then, the terms $\mathcal{T}_{\sigma}^{(i)}(x)$ in the classical equation of motion for the $\sigma$ field read:

$$
\begin{aligned}
& \mathcal{T}_{\sigma}^{(1)}(x) \equiv 6 \bar{\sigma}(x) \int \frac{\mathrm{d}^{3} \mathbf{k}}{(2 \pi)^{3}} e^{i \mathbf{k} \cdot \mathbf{x}} \mathcal{S}^{(1)}\left(x_{0}, \mathbf{k}\right) \\
& \mathcal{T}_{\sigma}^{(2)}(x) \equiv 4 \sum_{a} \bar{\pi}_{a}(x) \int \frac{\mathrm{d}^{3} \mathbf{k}}{(2 \pi)^{3}} e^{i \mathbf{k} \cdot \mathbf{x}} \mathcal{S}_{a}^{(2)}\left(x_{0}, \mathbf{k}\right) \\
& \mathcal{T}_{\sigma}^{(3)}(x) \equiv \int \frac{\mathrm{d}^{3} \mathbf{k}}{(2 \pi)^{3}} e^{i \mathbf{k} \cdot \mathbf{x}} \mathcal{S}^{(3)}\left(x_{0}, \mathbf{k}\right)
\end{aligned}
$$

where (for $\left.t_{i} \rightarrow-\infty\right)$ :

$$
\begin{aligned}
\mathcal{S}^{(1)}\left(x_{0}, \mathbf{k}\right) & \equiv \int \frac{\mathrm{d} \omega}{2 \pi} \mathcal{M}_{1}^{(\sigma)}(\omega, \mathbf{k}) \int \frac{\mathrm{d}^{3} \mathbf{p}}{(2 \pi)^{3}} \Theta\left(k_{c}-|\mathbf{p}|\right) \Theta\left(k_{c}-|\mathbf{k}-\mathbf{p}|\right) \\
& \times i \int_{0}^{\infty} \mathrm{d} \tau e^{-i \omega \tau}\left[3 \bar{\sigma}\left(x_{0}-\tau, \mathbf{k}-\mathbf{p}\right) \bar{\sigma}\left(x_{0}-\tau, \mathbf{p}\right)+\overline{\boldsymbol{\pi}}\left(x_{0}-\tau, \mathbf{k}-\mathbf{p}\right) \cdot \overline{\boldsymbol{\pi}}\left(x_{0}-\tau, \mathbf{p}\right)\right] \\
\mathcal{S}_{a}^{(2)}\left(x_{0}, \mathbf{k}\right) & \equiv \int \frac{\mathrm{d} \omega}{2 \pi} \mathcal{M}_{1}^{(\pi)}(\omega, \mathbf{k}) \int \frac{\mathrm{d}^{3} \mathbf{p}}{(2 \pi)^{3}} \Theta\left(k_{c}-|\mathbf{p}|\right) \Theta\left(k_{c}-|\mathbf{k}-\mathbf{p}|\right) \\
& \times i \int_{0}^{\infty} \mathrm{d} \tau e^{-i \omega \tau} \bar{\pi}_{a}\left(x_{0}-\tau, \mathbf{p}\right) \bar{\sigma}\left(x_{0}-\tau, \mathbf{k}-\mathbf{p}\right) \\
\mathcal{S}^{(3)}\left(x_{0}, \mathbf{k}\right) & \equiv 2 \int \frac{\mathrm{d} \omega}{2 \pi}\left[9 \mathcal{M}_{2}^{(\sigma \sigma)}(\omega, \mathbf{k})+(N-1) \mathcal{M}_{2}^{(\pi \pi)}(\omega, \mathbf{k})\right] \Theta\left(k_{c}-|\mathbf{k}|\right) i \int_{0}^{\infty} \mathrm{d} \tau e^{-i \omega \tau} \bar{\sigma}\left(x_{0}-\tau, \mathbf{k}\right) .
\end{aligned}
$$


In the linear harmonic approximation $\mathrm{A}$ ( ) these terms become:

$$
\begin{aligned}
& \mathcal{S}^{(1)}(t, \mathbf{k}) \simeq \int \frac{\mathrm{d}^{3} \mathbf{p}}{(2 \pi)^{3}} \Theta\left(k_{c}-|\mathbf{p}|\right) \Theta\left(k_{c}-|\mathbf{k}-\mathbf{p}|\right) \\
& \times\left\{-\frac{3}{2} \mathrm{P} \int \frac{\mathrm{d} \omega}{2 \pi} \mathcal{M}_{1}^{(\sigma)}(\omega, \mathbf{k})\left[\frac{1}{E_{1}^{(\sigma)}+E_{2}^{(\sigma)}-\omega}+\frac{1}{E_{1}^{(\sigma)}-E_{2}^{(\sigma)}-\omega}\right] \bar{\sigma}(t, \mathbf{p}) \bar{\sigma}(t, \mathbf{k}-\mathbf{p})\right. \\
& +\frac{3}{2 E_{1}^{(\sigma)} E_{2}^{(\sigma)}} \mathrm{P} \int \frac{\mathrm{d} \omega}{2 \pi} \mathcal{M}_{1}^{(\sigma)}(\omega, \mathbf{k})\left[\frac{1}{E_{1}^{(\sigma)}+E_{2}^{(\sigma)}-\omega}-\frac{1}{E_{1}^{(\sigma)}-E_{2}^{(\sigma)}-\omega}\right] \partial_{t} \bar{\sigma}(t, \mathbf{p}) \partial_{t} \bar{\sigma}(t, \mathbf{k}-\mathbf{p}) \\
& -\frac{1}{2} \mathrm{P} \int \frac{\mathrm{d} \omega}{2 \pi} \mathcal{M}_{1}^{(\sigma)}(\omega, \mathbf{k})\left[\frac{1}{E_{1}^{(\pi)}+E_{2}^{(\pi)}-\omega}+\frac{1}{E_{1}^{(\pi)}-E_{2}^{(\pi)}-\omega}\right] \overline{\boldsymbol{\pi}}(t, \mathbf{p}) \cdot \overline{\boldsymbol{\pi}}(t, \mathbf{k}-\mathbf{p}) \\
& +\frac{1}{2 E_{1}^{(\pi)} E_{2}^{(\pi)}} \mathrm{P} \int \frac{\mathrm{d} \omega}{2 \pi} \mathcal{M}_{1}^{(\sigma)}(\omega, \mathbf{k})\left[\frac{1}{E_{1}^{(\pi)}+E_{2}^{(\pi)}-\omega}-\frac{1}{E_{1}^{(\pi)}-E_{2}^{(\pi)}-\omega}\right] \partial_{t} \overline{\boldsymbol{\pi}}(t, \mathbf{p}) \cdot \partial_{t} \overline{\boldsymbol{\pi}}(t, \mathbf{k}-\mathbf{p}) \\
& -\frac{3}{2 E_{2}^{(\sigma)}}\left[\mathcal{M}_{1}^{(\sigma)}\left(E_{1}^{(\sigma)}+E_{2}^{(\sigma)}, \mathbf{k}\right)-\mathcal{M}_{1}^{(\sigma)}\left(E_{1}^{(\sigma)}-E_{2}^{(\sigma)}, \mathbf{k}\right)\right] \partial_{t} \bar{\sigma}(t, \mathbf{p}) \bar{\sigma}(t, \mathbf{k}-\mathbf{p}) \\
& \left.-\frac{1}{2 E_{2}^{(\pi)}}\left[\mathcal{M}_{1}^{(\sigma)}\left(E_{1}^{(\pi)}+E_{2}^{(\pi)}, \mathbf{k}\right)-\mathcal{M}_{1}^{(\sigma)}\left(E_{1}^{(\pi)}-E_{2}^{(\pi)}, \mathbf{k}\right)\right] \partial_{t} \overline{\boldsymbol{\pi}}(t, \mathbf{p}) \cdot \overline{\boldsymbol{\pi}}(t, \mathbf{k}-\mathbf{p})\right\}, \\
& \mathcal{S}_{a}^{(2)}(t, \mathbf{k}) \simeq \int \frac{\mathrm{d}^{3} \mathbf{p}}{(2 \pi)^{3}} \Theta\left(k_{c}-|\mathbf{p}|\right) \Theta\left(k_{c}-|\mathbf{k}-\mathbf{p}|\right) \\
& \times\left\{-\frac{1}{2} \mathrm{P} \int \frac{\mathrm{d} \omega}{2 \pi} \mathcal{M}_{1}^{(\pi)}(\omega, \mathbf{k})\left[\frac{1}{E_{1}^{(\sigma)}+E_{2}^{(\pi)}-\omega}+\frac{1}{E_{1}^{(\sigma)}-E_{2}^{(\pi)}-\omega}\right] \bar{\pi}_{a}(t, \mathbf{p}) \bar{\sigma}(t, \mathbf{k}-\mathbf{p})\right. \\
& +\frac{1}{2 E_{1}^{(\sigma)} E_{2}^{(\pi)}} \mathrm{P} \int \frac{\mathrm{d} \omega}{2 \pi} \mathcal{M}_{1}^{(\pi)}(\omega, \mathbf{k})\left[\frac{1}{E_{1}^{(\sigma)}+E_{2}^{(\pi)}-\omega}-\frac{1}{E_{1}^{(\sigma)}-E_{2}^{(\pi)}-\omega}\right] \partial_{t} \bar{\pi}_{a}(t, \mathbf{p}) \partial_{t} \bar{\sigma}(t, \mathbf{k}-\mathbf{p}) \\
& -\frac{1}{4 E_{1}^{(\sigma)}}\left[\mathcal{M}_{1}^{(\pi)}\left(E_{1}^{(\sigma)}+E_{2}^{(\pi)}, \mathbf{k}\right)+\mathcal{M}_{1}^{(\pi)}\left(E_{1}^{(\sigma)}-E_{2}^{(\pi)}, \mathbf{k}\right)\right] \bar{\pi}_{a}(t, \mathbf{p}) \partial_{t} \bar{\sigma}(t, \mathbf{k}-\mathbf{p}) \\
& \left.-\frac{1}{4 E_{2}^{(\pi)}}\left[\mathcal{M}_{1}^{(\pi)}\left(E_{1}^{(\sigma)}+E_{2}^{(\pi)}, \mathbf{k}\right)-\mathcal{M}_{1}^{(\pi)}\left(E_{1}^{(\sigma)}-E_{2}^{(\pi)}, \mathbf{k}\right)\right] \partial_{t} \bar{\pi}_{a}(t, \mathbf{p}) \bar{\sigma}(t, \mathbf{k}-\mathbf{p})\right\}, \\
& \mathcal{S}^{(3)}(t, \mathbf{k}) \simeq-2 \Theta\left(k_{c}-|\mathbf{k}|\right)\left[\mathrm{P} \int \frac{\mathrm{d} \omega}{2 \pi} \frac{9 \mathcal{M}_{2}^{(\sigma \sigma)}(\omega, \mathbf{k})+(N-1) \mathcal{M}_{2}^{(\pi \pi)}(\omega, \mathbf{k})}{E_{\mathbf{k}}^{(\sigma)}-\omega} \bar{\sigma}(t, \mathbf{k})\right. \\
& \left.+\frac{9 \mathcal{M}_{2}^{(\sigma \sigma)}\left(E_{\mathbf{k}}^{(\sigma)}, \mathbf{k}\right)+(N-1) \mathcal{M}_{2}^{(\pi \pi)}\left(E_{\mathbf{k}}^{(\sigma)}, \mathbf{k}\right)}{2 E_{\mathbf{k}}^{(\sigma)}} \partial_{t} \bar{\sigma}(t, \mathbf{k})\right],
\end{aligned}
$$

where $E_{1}^{(i)}=E_{\mathbf{k}-\mathbf{p}}^{(i)}$ and $E_{2}^{(i)}=E_{\mathbf{p}}^{(i)}$. The final expressions for $\mathcal{T}_{\sigma}^{(i)}(t, \mathbf{k})$ are:

$$
\begin{aligned}
& \mathcal{T}_{\sigma}^{(1)}(t, \mathbf{k}) \simeq 6 \int \frac{\mathrm{d}^{3} \mathbf{p}}{(2 \pi)^{3}} \Theta\left(k_{c}-|\mathbf{p}|\right) \bar{\sigma}(t, \mathbf{p}) \mathcal{S}^{(1)}(t, \mathbf{k}-\mathbf{p}), \\
& \mathcal{T}_{\sigma}^{(2)}(t, \mathbf{k}) \simeq 4 \int \frac{\mathrm{d}^{3} \mathbf{p}}{(2 \pi)^{3}} \Theta\left(k_{c}-|\mathbf{p}|\right) \sum_{a} \bar{\pi}_{a}(t, \mathbf{p}) \mathcal{S}_{a}^{(2)}(t, \mathbf{k}-\mathbf{p}), \\
& \mathcal{T}_{\sigma}^{(3)}(t, \mathbf{k}) \simeq \mathcal{S}^{(3)}(t, \mathbf{k}) .
\end{aligned}
$$

Since $\mathcal{M}_{1}^{(i)}(\omega, \mathbf{0}) \equiv 0$, cf. eq. (C2a), it follows from relations $\mathrm{C} 5$ and $(\mathrm{C} 6)$ that for $k_{c} \rightarrow 0, \mathcal{T}_{\sigma}^{(1)}(t, \mathbf{0})=$ $\mathcal{T}_{\sigma}^{(2)}(t, \mathbf{0}) \equiv 0$. 


\section{APPENDIX D: THE INTERACTION TERMS IN THE EQUATION OF MOTION FOR THE $\pi$ FIELD}

The interaction terms in the equation of motion for the $\bar{\pi}$ field are:

$$
\begin{aligned}
& \mathcal{T}_{\pi_{a}}^{(1)}(x) \equiv 2 \bar{\pi}_{a}(x) \int \frac{\mathrm{d}^{3} \mathbf{k}}{(2 \pi)^{3}} e^{i \mathbf{k} \cdot \mathbf{x}} \mathcal{P}^{(1)}\left(x_{0}, \mathbf{k}\right), \\
& \mathcal{T}_{\pi_{a}}^{(2)}(x) \equiv 4 \bar{\sigma}(x) \int \frac{\mathrm{d}^{3} \mathbf{k}}{(2 \pi)^{3}} e^{i \mathbf{k} \cdot \mathbf{x}} \mathcal{P}_{a}^{(2)}\left(x_{0}, \mathbf{k}\right) \\
& \mathcal{T}_{\pi_{a}}^{(3)}(x) \equiv \int \frac{\mathrm{d}^{3} \mathbf{k}}{(2 \pi)^{3}} e^{i \mathbf{k} \cdot \mathbf{x}} \mathcal{P}_{a}^{(3)}\left(x_{0}, \mathbf{k}\right)
\end{aligned}
$$

where $\mathcal{P}^{(1)}\left(x_{0}, \mathbf{k}\right) \equiv \mathcal{S}^{(1)}\left(x_{0}, \mathbf{k}\right)$, and $\mathcal{P}_{a}^{(2)}\left(x_{0}, \mathbf{k}\right) \equiv \mathcal{S}_{a}^{(2)}\left(x_{0}, \mathbf{k}\right)$, cf. eqs. C4a and (C4b), while

$$
\mathcal{P}_{a}^{(3)}\left(x_{0}, \mathbf{k}\right) \equiv 4 \int \frac{\mathrm{d} \omega}{2 \pi} \mathcal{M}_{2}^{(\sigma \pi)}(\omega, \mathbf{k}) \Theta\left(k_{c}-|\mathbf{k}|\right) i \int_{0}^{\infty} \mathrm{d} \tau e^{-i \omega \tau} \bar{\pi}_{a}\left(x_{0}-\tau, \mathbf{k}\right) .
$$

In linear harmonic approximation we therefore obtain:

$$
\begin{aligned}
& \mathcal{T}_{\pi_{a}}^{(1)}(t, \mathbf{k}) \simeq 2 \int \frac{\mathrm{d}^{3} \mathbf{p}}{(2 \pi)^{3}} \Theta\left(k_{c}-|\mathbf{p}|\right) \bar{\pi}_{a}(t, \mathbf{p}) \mathcal{S}^{(1)}(t, \mathbf{k}-\mathbf{p}), \\
& \mathcal{T}_{\pi_{a}}^{(2)}(t, \mathbf{k}) \simeq 4 \int \frac{\mathrm{d}^{3} \mathbf{p}}{(2 \pi)^{3}} \Theta\left(k_{c}-|\mathbf{p}|\right) \bar{\sigma}(t, \mathbf{p}) \mathcal{S}_{a}^{(2)}(t, \mathbf{k}-\mathbf{p}), \\
& \mathcal{T}_{\pi_{a}}^{(3)}(t, \mathbf{k}) \simeq-4 \Theta\left(k_{c}-|\mathbf{k}|\right)\left[\mathrm{P} \int \frac{\mathrm{d} \omega}{2 \pi} \frac{\mathcal{M}_{2}^{(\sigma \pi)}(\omega, \mathbf{k})}{E_{\mathbf{k}}^{(\pi)}-\omega} \bar{\pi}_{a}(t, \mathbf{k})+\frac{\mathcal{M}_{2}^{(\sigma \pi)}\left(E_{\mathbf{k}}^{(\pi)}, \mathbf{k}\right)}{2 E_{\mathbf{k}}^{(\pi)}} \partial_{t} \bar{\pi}_{a}(t, \mathbf{k})\right] .
\end{aligned}
$$

Again, since $\mathcal{M}_{1}^{(i)}(\omega, \mathbf{0}) \equiv 0$, cf. eq. (A2a), it follows for $k_{c} \rightarrow 0$ that $\mathcal{T}_{\pi_{a}}^{(1)}(t, \mathbf{0})=\mathcal{T}_{\pi_{a}}^{(2)}(t, \mathbf{0}) \equiv 0$.

\section{APPENDIX E: THE DISSIPATION COEFFICIENT FOR THE $\sigma$ FIELD}

The dissipation coefficient $\eta_{\sigma}$ in the classical equation of motion for $\bar{\sigma}(t)$, eq. (78) is defined by

$$
\eta_{\sigma} \equiv\left(\frac{4 \lambda f_{\pi}}{N}\right)^{2} 2 \frac{9 \mathcal{M}_{2}^{(\sigma \sigma)}\left(m_{\sigma}, \mathbf{0}\right)+(N-1) \mathcal{M}_{2}^{(\pi \pi)}\left(m_{\sigma}, \mathbf{0}\right)}{2 m_{\sigma}}
$$

where $\mathcal{M}_{2}^{(i j)}(\omega, \mathbf{k})$ is given by eq. (C2b). $\mathcal{M}_{2}^{(\sigma \sigma)}\left(m_{\sigma}, \mathbf{0}\right)$ describes the decay of a $\sigma$ at rest into two $\sigma$ 's (and the reverse reaction, corresponding to the second and third line of eq. (C2b) , and the absorption of a $\sigma$ by a $\sigma$ at rest, producing a $\sigma$ (corresponding to the fourth and fifth line of eq. (C2b)).

However, a $\sigma$ at rest cannot decay into two $\sigma$ 's by energy conservation (similarly, two $\sigma$ 's cannot annihilate to form one), and also the absorption of a $\sigma$ by a $\sigma$ at rest, producing a $\sigma$, is also impossible. Therefore, $\mathcal{M}_{2}^{(\sigma \sigma)}\left(m_{\sigma}, \mathbf{0}\right) \equiv 0$. Mathematically, this is immediately obvious inspecting the arguments of the $\delta$ functions in eq. (C2b) with $\omega \equiv m_{\sigma}, \mathbf{k}=0$.

Similar arguments prevent the absorption of a $\pi$ by a $\sigma$ at rest, producing a $\pi$, but the decay of a heavy $\sigma$ at rest into two light $\pi$ 's (and the corresponding reverse reaction of $\pi \pi \rightarrow \sigma$ ) is always possible (provided $m_{\sigma}>2 m_{\pi}$ ). Therefore (utilizing detailed balance, and taking the limit $k_{c} \rightarrow 0$ ),

$$
\begin{aligned}
\mathcal{M}_{2}^{(\pi \pi)}\left(m_{\sigma}, \mathbf{0}\right) & =2 \pi\left(1-\exp \left[-m_{\sigma} / T\right]\right) \int \frac{\mathrm{d}^{3} \mathbf{k}}{(2 \pi)^{3}}\left[2 E_{\mathbf{k}}^{(\pi)}\right]^{-2}\left[1+n\left(E_{\mathbf{k}}^{(\pi)}\right)\right]^{2} \delta\left(m_{\sigma}-2 E_{\mathbf{k}}^{(\pi)}\right) \\
& =\frac{1}{8 \pi} \sqrt{1-\frac{4 m_{\pi}^{2}}{m_{\sigma}^{2}}} \frac{1+\exp \left[-m_{\sigma} / 2 T\right]}{1-\exp \left[-m_{\sigma} / 2 T\right]} .
\end{aligned}
$$

Note that this expression is non-zero even at vanishing temperature. The dissipation coefficient becomes:

$$
\eta_{\sigma}=\left(\frac{4 \lambda f_{\pi}}{N}\right)^{2} \frac{N-1}{8 \pi m_{\sigma}} \sqrt{1-\frac{4 m_{\pi}^{2}}{m_{\sigma}^{2}}} \operatorname{coth} \frac{m_{\sigma}}{4 T} .
$$




\section{APPENDIX F: THE DISSIPATION COEFFICIENT FOR THE $\pi$ FIELD}

The dissipation coefficient $\eta_{\pi}$ in the classical equation of motion for $\bar{\pi}_{a}(t)$, eq. (82), is defined by

$$
\eta_{\pi} \equiv\left(\frac{4 \lambda f_{\pi}}{N}\right)^{2} 4 \frac{\mathcal{M}_{2}^{(\sigma \pi)}\left(m_{\pi}, \mathbf{0}\right)}{2 m_{\pi}}
$$

where $\mathcal{M}_{2}^{(i j)}(\omega, \mathbf{k})$ is the Fourier transform of $\mathcal{M}_{2}^{(i j)}(x)$, eq. $(\mathrm{C} 2 \mathrm{~b}) . \mathcal{M}_{2}^{(\sigma \pi)}\left(m_{\pi}, \mathbf{0}\right)$ describes the decay of a $\pi$ at rest into a $\pi$ and a $\sigma$ (and the reverse reaction, corresponding to the second and third line of eq. (C2b)), and the absorption of a $\pi$ or $\sigma$ by a $\pi$ at rest, producing a $\sigma$ or a $\pi$, respectively (corresponding to the fourth and fifth line of eq. $(\mathrm{C} 2 \mathrm{~b})$ ).

The decay of a $\pi$ into $\sigma$ and $\pi$ and the reverse process is kinematically forbidden. The remaining contribution reads with detailed balance (and taking the limit $k_{c} \rightarrow 0$ ):

$$
\begin{aligned}
\mathcal{M}_{2}^{(\sigma \pi)}\left(m_{\pi}, \mathbf{0}\right) & =2 \pi\left(1-\exp \left[-m_{\pi} / T\right]\right) \int \frac{\mathrm{d}^{3} \mathbf{k}}{(2 \pi)^{3}} \frac{1+n\left(E_{\mathbf{k}}^{(\sigma)}\right)}{2 E_{\mathbf{k}}^{(\sigma)}} \frac{n\left(E_{\mathbf{k}}^{(\pi)}\right)}{2 E_{\mathbf{k}}^{(\pi)}} \delta\left(E_{\mathbf{k}}^{(\pi)}+m_{\pi}-E_{\mathbf{k}}^{(\sigma)}\right) \\
& =\frac{1}{8 \pi} \frac{m_{\sigma}^{2}}{m_{\pi}^{2}} \sqrt{1-\frac{4 m_{\pi}^{2}}{m_{\sigma}^{2}}} \frac{1-\exp \left[-m_{\pi} / T\right]}{1-\exp \left[-m_{\sigma}^{2} / 2 m_{\pi} T\right]} \frac{1}{\exp \left[\left(m_{\sigma}^{2}-2 m_{\pi}^{2}\right) / 2 m_{\pi} T\right]-1} .
\end{aligned}
$$

The dissipation coefficient therefore becomes

$$
\eta_{\pi}=\left(\frac{4 \lambda f_{\pi}}{N}\right)^{2} \frac{m_{\sigma}^{2}}{4 \pi m_{\pi}^{3}} \sqrt{1-\frac{4 m_{\pi}^{2}}{m_{\sigma}^{2}}} \frac{1-\exp \left[-m_{\pi} / T\right]}{1-\exp \left[-m_{\sigma}^{2} / 2 m_{\pi} T\right]} \frac{1}{\exp \left[\left(m_{\sigma}^{2}-2 m_{\pi}^{2}\right) / 2 m_{\pi} T\right]-1} .
$$

This expression vanishes at $T=0$ and in the limit $m_{\pi} \rightarrow 0$.

\section{APPENDIX G: THE VARIANCES OF THE NOISE FIELDS}

The variances of the noise fields are given by eqs. (75). In order to evaluate them, let us define in analogy to eqs. (C1) the "noise kernels"

$$
\begin{aligned}
\mathcal{N}_{1}^{(i)}(x) & \equiv D_{>}^{(i)}(x)+D_{<}^{(i)}(x), \\
\mathcal{N}_{2}^{(i j)}(x) & \equiv D_{>}^{(i)}(x) D_{>}^{(i)}(x)+D_{<}^{(i)}(x) D_{<}^{(i)}(x), \quad i, j=\sigma \quad \text { or } \pi
\end{aligned}
$$

Their Fourier transforms are quite similar to those of $\mathcal{M}_{1}^{(i)}$ and $\mathcal{M}_{2}^{(i j)}$ :

$$
\begin{aligned}
& \mathcal{N}_{1}^{(i)}(\omega, \mathbf{k})= \Theta\left(|\mathbf{k}|-k_{c}\right) \frac{2 \pi}{2 E_{\mathbf{k}}^{(i)}}\left[1+2 n\left(E_{\mathbf{k}}^{(i)}\right)\right]\left[\delta\left(\omega-E_{\mathbf{k}}^{(i)}\right)+\delta\left(\omega+E_{\mathbf{k}}^{(i)}\right)\right], \\
& \mathcal{N}_{2}^{(i j)}(\omega, \mathbf{k})=2 \pi \int \frac{\mathrm{d}^{3} \mathbf{p}}{(2 \pi)^{3}} \Theta\left(|\mathbf{p}|-k_{c}\right) \Theta\left(|\mathbf{k}-\mathbf{p}|-k_{c}\right) \frac{1}{4 E_{\mathbf{p}}^{(i)} E_{\mathbf{k}-\mathbf{p}}^{(j)}}, \\
& \times\{( {\left.\left[1+n\left(E_{\mathbf{p}}^{(i)}\right)\right]\left[1+n\left(E_{\mathbf{k}-\mathbf{p}}^{(j)}\right)\right]+n\left(E_{\mathbf{p}}^{(i)}\right) n\left(E_{\mathbf{k}-\mathbf{p}}^{(j)}\right)\right) } \\
& \times\left[\delta\left(\omega-E_{\mathbf{p}}^{(i)}-E_{\mathbf{k}-\mathbf{p}}^{(j)}\right)+\delta\left(\omega+E_{\mathbf{p}}^{(i)}+E_{\mathbf{k}-\mathbf{p}}^{(j)}\right)\right] \\
&+\left(\left[1+n\left(E_{\mathbf{p}}^{(i)}\right)\right] n\left(E_{\mathbf{k}-\mathbf{p}}^{(j)}\right)+n\left(E_{\mathbf{p}}^{(i)}\right)\left[1+n\left(E_{\mathbf{k}-\mathbf{p}}^{(j)}\right)\right]\right) \\
&\left.\times\left[\delta\left(\omega-E_{\mathbf{p}}^{(i)}+E_{\mathbf{k}-\mathbf{p}}^{(j)}\right)+\delta\left(\omega+E_{\mathbf{p}}^{(i)}-E_{\mathbf{k}-\mathbf{p}}^{(j)}\right)\right]\right\} .
\end{aligned}
$$

Using the fact that the hard modes are distributed according to the Bose-Einstein distribution function $n(E)=\left(e^{E / T}-1\right)^{-1}$, one can show that (cf. eq. (66) of [8]) 


$$
\begin{aligned}
\mathcal{N}_{1}^{(i)}(\omega, \mathbf{k}) & \equiv \mathcal{M}_{1}^{(i)}(\omega, \mathbf{k}) \operatorname{coth}\left[\frac{\omega}{2 T}\right] \\
\mathcal{N}_{2}^{(i j)}(\omega, \mathbf{k}) & \equiv \mathcal{M}_{2}^{(i j)}(\omega, \mathbf{k}) \operatorname{coth}\left[\frac{\omega}{2 T}\right] .
\end{aligned}
$$

For the Fourier transforms of $\mathcal{I}_{a b}(x, y)$ one therefore derives $\left(t_{i} \rightarrow-\infty, t_{f} \rightarrow+\infty\right)$ :

$$
\begin{aligned}
\mathcal{I}_{\sigma \sigma}\left(k_{0}, \mathbf{k} ; q_{0}, \mathbf{q}\right) \equiv & \int_{t_{i}}^{t_{f}} \mathrm{~d}^{4} x \mathrm{~d}^{4} y e^{i\left(k_{0} x_{0}-\mathbf{k} \cdot \mathbf{x}+q_{0} y_{0}-\mathbf{q} \cdot \mathbf{y}\right)} \mathcal{I}_{\sigma \sigma}(x, y) \\
= & \left(\frac{4 \lambda f_{\pi}}{N}\right)^{2}\left\{\int \frac{\mathrm{d} p_{0} \mathrm{~d}^{3} \mathbf{p}}{(2 \pi)^{4}} \Theta\left(k_{c}-|\mathbf{p}|\right) \Theta\left(k_{c}-|\mathbf{k}+\mathbf{q}-\mathbf{p}|\right)\right. \\
& \times\left[18 \bar{\sigma}\left(p_{0}, \mathbf{p}\right) \mathcal{N}_{1}^{(\sigma)}\left(k_{0}-p_{0}, \mathbf{k}-\mathbf{p}\right) \bar{\sigma}\left(k_{0}+q_{0}-p_{0}, \mathbf{k}+\mathbf{q}-\mathbf{p}\right)\right. \\
& \left.+2 \sum_{a} \bar{\pi}_{a}\left(p_{0}, \mathbf{p}\right) \mathcal{N}_{1}^{(\pi)}\left(k_{0}-p_{0}, \mathbf{k}-\mathbf{p}\right) \bar{\pi}_{a}\left(k_{0}+q_{0}-p_{0}, \mathbf{k}+\mathbf{q}-\mathbf{p}\right)\right] \\
& \left.+(2 \pi)^{4} \delta\left(k_{0}+q_{0}\right) \delta^{(3)}(\mathbf{k}+\mathbf{q})\left[9 \mathcal{N}_{2}^{(\sigma \sigma)}\left(k_{0}, \mathbf{k}\right)+(N-1) \mathcal{N}_{2}^{(\pi \pi)}\left(k_{0}, \mathbf{k}\right)\right]\right\}, \\
\mathcal{I}_{\sigma \pi_{a}}\left(k_{0}, \mathbf{k} ; q_{0}, \mathbf{q}\right)= & \left(\frac{4 \lambda f_{\pi}}{N}\right)^{2} \int \frac{\mathrm{d} p_{0} \mathrm{~d}^{3} \mathbf{p}}{(2 \pi)^{4}} \Theta\left(k_{c}-|\mathbf{p}|\right) \Theta\left(k_{c}-|\mathbf{k}+\mathbf{q}-\mathbf{p}|\right) \\
& \times\left[6 \bar{\sigma}\left(p_{0}, \mathbf{p}\right) \mathcal{N}_{1}^{(\sigma)}\left(k_{0}-p_{0}, \mathbf{k}-\mathbf{p}\right) \bar{\pi}_{a}\left(k_{0}+q_{0}-p_{0}, \mathbf{k}+\mathbf{q}-\mathbf{p}\right)\right. \\
& \left.+2 \bar{\pi}_{a}\left(p_{0}, \mathbf{p}\right) \mathcal{N}_{1}^{(\pi)}\left(k_{0}-p_{0}, \mathbf{k}-\mathbf{p}\right) \bar{\sigma}\left(k_{0}+q_{0}-p_{0}, \mathbf{k}+\mathbf{q}-\mathbf{p}\right)\right] \\
\mathcal{I}_{\pi_{a} \pi_{b}}\left(k_{0}, \mathbf{k} ; q_{0}, \mathbf{q}\right)= & \left(\frac{4 \lambda f_{\pi}}{N}\right)^{2}\left\{\int \frac{\mathrm{d} p_{0} \mathrm{~d}^{3} \mathbf{p}}{(2 \pi)^{4}} \Theta\left(k_{c}-|\mathbf{p}|\right) \Theta\left(k_{c}-|\mathbf{k}+\mathbf{q}-\mathbf{p}|\right)\right. \\
& \times\left[2 \bar{\pi}_{a}\left(p_{0}, \mathbf{p}\right) \mathcal{N}_{1}^{(\sigma)}\left(k_{0}-p_{0}, \mathbf{k}-\mathbf{p}\right) \bar{\pi}_{b}\left(k_{0}+q_{0}-p_{0}, \mathbf{k}+\mathbf{q}-\mathbf{p}\right)\right. \\
& \left.+2 \delta_{a b} \bar{\sigma}\left(p_{0}, \mathbf{p}\right) \mathcal{N}_{1}^{(\pi)}\left(k_{0}-p_{0}, \mathbf{k}-\mathbf{p}\right) \bar{\sigma}\left(k_{0}+q_{0}-p_{0}, \mathbf{k}+\mathbf{q}-\mathbf{p}\right)\right] \\
& \left.+2 \delta_{a b}(2 \pi)^{4} \delta\left(k_{0}+q_{0}\right) \delta^{(3)}(\mathbf{k}+\mathbf{q}) \mathcal{N}_{2}^{(\sigma \pi)}\left(k_{0}, \mathbf{k}\right)\right\}
\end{aligned}
$$

The focus of interest are the spatially homogeneous noise terms, $\mathbf{k}=\mathbf{q}=0$. For this case, all integrals vanish in these expressions due to the fact that $\mathcal{N}_{1}^{(i)}(\omega, \mathbf{k})$ is proportional to $\Theta\left(|\mathbf{k}|-k_{c}\right)$, cf. eq. (G2a). This has the further consequence that all cross correlations between the noise fields vanish, $\mathcal{I}_{a b} \sim \delta_{a b}$. Using $(2 \pi)^{3} \delta^{(3)}(\mathbf{k}+\mathbf{q}) \equiv V \delta_{\mathbf{k}+\mathbf{q}, 0}^{(3)}$ one arrives at:

$$
\begin{aligned}
\mathcal{I}_{\sigma \sigma}\left(k_{0}, \mathbf{0} ; q_{0}, \mathbf{0}\right) & =V 2 \pi \delta\left(k_{0}+q_{0}\right)\left(\frac{4 \lambda f_{\pi}}{N}\right)^{2}\left[9 \mathcal{N}_{2}^{(\sigma \sigma)}\left(k_{0}, \mathbf{0}\right)+(N-1) \mathcal{N}_{2}^{(\pi \pi)}\left(k_{0}, \mathbf{0}\right)\right], \\
\mathcal{I}_{\pi_{a} \pi_{b}}\left(k_{0}, \mathbf{0} ; q_{0}, \mathbf{0}\right) & =\delta_{a b} V 2 \pi \delta\left(k_{0}+q_{0}\right)\left(\frac{4 \lambda f_{\pi}}{N}\right)^{2} 2 \mathcal{N}_{2}^{(\sigma \pi)}\left(k_{0}, \mathbf{0}\right) .
\end{aligned}
$$

For the variance of the noise fields $\xi_{\sigma}(t)$ and $\xi_{\pi_{a}}(t)$ one therefore obtains:

$$
\begin{aligned}
\left\langle\xi_{\sigma}(t) \xi_{\sigma}\left(t^{\prime}\right)\right\rangle_{\xi} & \equiv\left\langle\frac{\xi_{\sigma}(t, \mathbf{0})}{V} \frac{\xi_{\sigma}\left(t^{\prime}, \mathbf{0}\right)}{V}\right\rangle_{\xi}=\int \frac{\mathrm{d} k_{0} \mathrm{~d} q_{0}}{(2 \pi)^{2}} e^{i\left(k_{0} t+q_{0} t^{\prime}\right)}\left\langle\frac{\xi_{\sigma}\left(-k_{0}, \mathbf{0}\right)}{V} \frac{\xi_{\sigma}\left(-q_{0}, \mathbf{0}\right)}{V}\right\rangle_{\xi} \\
& =\int \frac{\mathrm{d} k_{0} \mathrm{~d} q_{0}}{(2 \pi)^{2}} e^{i\left(k_{0} t+q_{0} t^{\prime}\right)} \frac{1}{V^{2}} \mathcal{I}_{\sigma \sigma}\left(k_{0}, \mathbf{0} ; q_{0}, \mathbf{0}\right) \\
& =\int \frac{\mathrm{d} k_{0}}{2 \pi} e^{i k_{0}\left(t-t^{\prime}\right)} \frac{1}{V}\left(\frac{4 \lambda f_{\pi}}{N}\right)^{2}\left[9 \mathcal{N}_{2}^{(\sigma \sigma)}\left(k_{0}, \mathbf{0}\right)+(N-1) \mathcal{N}_{2}^{(\pi \pi)}\left(k_{0}, \mathbf{0}\right)\right], \\
\left\langle\xi_{\pi_{a}}(t) \xi_{\pi_{b}}\left(t^{\prime}\right)\right\rangle_{\xi} & =\delta_{a b} \int \frac{\mathrm{d} k_{0}}{2 \pi} e^{i k_{0}\left(t-t^{\prime}\right)} \frac{1}{V}\left(\frac{4 \lambda f_{\pi}}{N}\right)^{2} 2 \mathcal{N}_{2}^{(\sigma \pi)}\left(k_{0}, \mathbf{0}\right) .
\end{aligned}
$$


Here, $\langle\cdot\rangle_{\xi}$ is the average with respect to the Gaussian measure (29). Further evaluation is simplified by approximating $\mathcal{N}_{2}^{(\sigma \sigma)}\left(k_{0}, \mathbf{0}\right) \simeq \mathcal{N}_{2}^{(\sigma \sigma)}\left(m_{\sigma}, \mathbf{0}\right), \mathcal{N}_{2}^{(\pi \pi)}\left(k_{0}, \mathbf{0}\right) \simeq \mathcal{N}_{2}^{(\pi \pi)}\left(m_{\sigma}, \mathbf{0}\right), \mathcal{N}_{2}^{(\sigma \pi)}\left(k_{0}, \mathbf{0}\right) \simeq \mathcal{N}_{2}^{(\sigma \pi)}\left(m_{\pi}, \mathbf{0}\right)$, i.e., taking the energy $k_{0}$ to be the on-shell energy (i.e., since $\mathbf{k}=0$ for both $\sigma$ and $\pi$ fields, the mass) of the respective particle. This approximation is consistent with the linear harmonic approximation which puts the energy in the $\mathcal{M}_{2}$ functions on-shell. The consequence is that the $k_{0}$-integral can be performed, yielding with eqs. (G3), (E1), and (F1):

$$
\begin{aligned}
\left\langle\xi_{\sigma}(t) \xi_{\sigma}\left(t^{\prime}\right)\right\rangle_{\xi} & \simeq \delta\left(t-t^{\prime}\right) \frac{1}{V} m_{\sigma} \eta_{\sigma} \operatorname{coth}\left[\frac{m_{\sigma}}{2 T}\right], \\
\left\langle\xi_{\pi_{a}}(t) \xi_{\pi_{b}}\left(t^{\prime}\right)\right\rangle_{\xi} & \simeq \delta_{a b} \delta\left(t-t^{\prime}\right) \frac{1}{V} m_{\pi} \eta_{\pi} \operatorname{coth}\left[\frac{m_{\pi}}{2 T}\right],
\end{aligned}
$$

The $\delta$ function corresponds to white noise. Therefore, this approximation will be called "white-noise" approximation.

[1] see for instance: E. Laermann, Nucl. Phys. A610, 1c (1996).

[2] see for instance: J.W. Harris and B. Müller, Annu. Rev. Nucl. Part. Sci. 46, 71 (1996).

[3] A.A. Anselm, Phys. Lett. B217, 169 (1989); A.A. Anselm and M.G. Ryskin, Phys. Lett. B266, 482 (1991); J.D. Bjorken, Int. J. Mod. Phys. A 7, 4189 (1992); J.-P. Blaizot and A. Krzywicki, Phys. Rev. D 46, 246 (1992); K. Rajagopal and F. Wilczek, Nucl. Phys. B399, 395 (1993); J.D. Bjorken, K.L. Kowalski, and C.C. Taylor, SLAC report no. SLAC-PUB-6109 (1993); K. Rajagopal, invited talk and proceedings of the Int. Workshop on QCD Phase Transitions, Hirschegg, Austria, January 1997, hep-ph/9703258.

[4] K. Rajagopal and F. Wilczek, Nucl. Phys. B404, 577 (1993); M. Asakawa, Z. Huang, and X.N. Wang, Phys. Rev. Lett. 74, 3126 (1995).

[5] S. Gavin and B. Müller, Phys. Lett. B329, 486 (1994); S. Mrowczynski and B. Müller, Phys. Lett. B363, 1 (1995); T.S. Biro, D. Molnar, Z. Feng, and L.P. Csernai, Phys. Rev. D 55, 6900 (1997); J. Randrup, Nucl. Phys. A616, 531 (1997); M. Asakawa, H. Minakata, and B. Müller, preprint DPNU-98-04 and nucl-th/9802006; for a review, see: K. Rajagopal, in: Quark-Gluon Plasma 2, (ed. R. Hwa, World Scientific, 1995), p. 484.

[6] see for instance: M. Bleicher et al., Proceedings of Quark Matter '97, Thirteenth International Conference on Ultra-Relativistic Nucleus-Nucleus Collisions, Tsukuba, Japan, December 1-5, 1997, to appear in Nucl. Phys. A.

[7] T. Biro and C. Greiner, Phys. Rev. Lett. 79, 3138 (1997).

[8] C. Greiner and B. Müller, Phys. Rev. D 55, 1026 (1997).

[9] C. Greiner and S. Leupold, preprint UGI-98-12 and hep-ph/9802312.

[10] R.P. Feynman and A.R. Hibbs, Quantum Mechanics and Path Integrals, (McGraw-Hill, New York, 1965).

[11] D. Boyanovsky, H.J. de Vega, R. Holman, D.-S. Lee, and A. Singh, Phys. Rev. D 51, 4419 (1995).

[12] D. Bödecker, L. McLerran, and A. Smilga, Phys. Rev. D 52, 4675 (1995); F. Lombardo and F.D. Mazzitelli, Phys. Rev. D 53, 2001 (1996).

[13] D.T. Son, University of Washington preprint UW/PT-97-19 and hep-ph/9707351.

[14] M. Gleiser and R. Ramos, Phys. Rev. D 50, 2441 (1994).

[15] C.M. Lattes, Y. Fujimoto, and S. Hasegawa, Phys. Rep. 65, 151 (1980).

[16] K. Rajagopal and D.H. Rischke, in preparation.

[17] D.H. Rischke, in preparation.

[18] J. Schwinger, J. Math. Phys. 2, 407 (1961); L.V. Keldysh, Zh. Eksp. Teor. Fiz. 47, 1515 (1964) [Sov. Phys. JETP 20, 1018 (1964)].

[19] see for instance: M. LeBellac, Thermal Field Theory, (Cambridge University Press, Cambridge, 1996).

[20] S. Jeon, Phys. Rev. D 52, 3591 (1995); E. Wang and U. Heinz, Phys. Rev. D 53, 899 (1996).

[21] A. Bochkarev and J. Kapusta, Phys. Rev. D 54, 4066 (1996).

[22] H.A. Weldon, Phys. Rev. D 28, 2007 (1983).

[23] R.D. Pisarski and M. Tytgat, talk given at the 2nd Workshop on Continuous Advances in QCD, Minneapolis, March 28-31, 1996, hep-ph/9606459.

[24] Y. Abe, S. Ayik, P.G. Reinhard, and E. Suraud, Phys. Rep. 275, 49 (1996).

[25] J. Randrup, Phys. Rev. Lett. 77, 1226 (1996). 